\title{
O teorema do índice para o círculo
}

\author{
Rodrigo Ferraz de Andrade
}

DisSERTAÇÃO APRESENTADA

$\mathrm{AO}$

Instituto de Matemática E Estatística

DA

Universidade DE SÃo PaUlo

PARA

OBTENÇÃO DO TÍTULO

DE

Mestre eM CiênCIAS

\author{
Programa: Matemática \\ Orientador: Prof. Dr. Severino Toscano do Rêgo Melo
}

Durante o desenvolvimento deste trabalho o autor recebeu auxílio financeiro do $\mathrm{CNPq}$

São Paulo, agosto de 2008 


\section{O teorema do índice para o círculo}

Este exemplar corresponde à redação final da dissertação devidamente corrigida e defendida por Rodrigo Ferraz de Andrade e aprovada pela Comissão Julgadora.

Banca Examinadora:

- Prof. Dr. Severino Toscano do Rêgo Melo (orientador) - IME-USP.

- Prof. Dr. Daniel Victor Tausk - IME-USP.

- Prof. Dr. José Ruidival Soares dos Santos Filho - UFS-

Car. 


\section{Agradecimentos}

Agradeço ao meu orientador, Professor Toscano, ao Daniel V. Tausk e à Mariana por toda a ajuda, paciência e disposição para ouvir e discutir. Sou muito grato também à Da. Johanna W. Smit, cujo apoio me foi indispensável durante os meus anos de graduação e mestrado. 


\section{Resumo}

Estudamos a álgebra de comparação do círculo, descrevemos o espaço de Gelfand do seu quociente pelos compactos e damos uma fórmula para o índice dos seus operadores de Fredholm. Depois generalizamos o resultado para as matrizes com elementos na álgebra de comparação e damos uma aplicação para operadores diferenciais no círculo.

Palavras-chave: Álgebra de Comparação, Teoria do índice de Fredholm, Espaço de Gelfand. 


\section{Abstract}

We study the comparison algebra on the circle, we describe the Gelfand space of its quotient by the compacts and we give a formula to compute the index of its Fredholm operators. After that, we generalize the result to the matrices with entries in the comparison algebra and give an application to differential opperators in the circle.

Keywords: Comparison Algebra, Theory of the Fredholm index, Gelfand Space. 


\section{SUMÁRIO}

1. Introdução 1

2. Algumas Definições e Notações 9

3. Roteiro da Dissertação 14

4. Os Comutadores de $\mathcal{A}$ são Compactos 18

5. Os Operadores Compactos estão em $\mathcal{A} \quad 21$

6. $\mathcal{A} / \mathcal{K}$ é uma $C^{*}$-Álgebra Comutativa 25

7. $M_{\mathcal{A} / \mathcal{K}}$ é Homeomorfo à União Disjunta de Duas Cópias de $S^{1} \quad 27$

8. O Índice de Fredholm de $T \in \mathcal{A}$ a partir do seu Símbolo $\quad 38$

9. $T \in M_{n}(\mathcal{A})$ é de Fredholm se, e só se, seu Símbolo é Inversível 43

10. O Índice de $A \in \mathcal{F}\left(M_{n}(\mathcal{A})\right)$ a partir do Símbolo de $A$

11. Uma Aplicação aos Operadores Diferenciais 49

Apêndice A. O Lema Sinal $\quad 55$

Apêndice B. O Levantamento de um Caminho em $\mathcal{A} / \mathcal{J}$

Apêndice C. Solução do Problema de Valor de Contorno 66

Apêndice D. Redes 71

$\begin{array}{lll}\text { Apêndice E. } & \text { Lema de Topologia Algébrica } & 77\end{array}$

Referências $\quad 83$ 


\section{INTRODUÇÃO}

Sejam $\mathcal{M}$ uma variedade sem bordo compacta de dimensão $n$ e $G$ uma métrica riemanniana em $\mathcal{M}$, que, para cada $x \in \mathcal{M}$, associa a $\left(x, T_{x} \mathcal{M}\right) \mathrm{um}$ produto interno $\langle\cdot, \cdot\rangle_{x}$ definido em $T_{x} \mathcal{M}$. Definimos então o operador de Laplace-Beltrami:

$$
\begin{aligned}
\Delta: C^{\infty}(\mathcal{M}) & \longrightarrow C^{\infty}(\mathcal{M}) \\
f & \longmapsto \Delta f
\end{aligned}
$$

onde, se $(V, \chi)$ é uma carta para o ponto $x \in \mathcal{M}$ e $\tilde{f}:=f \circ \chi^{-1}$, e se $\langle\cdot, \cdot\rangle_{x}$ na base de $T_{x} \mathcal{M}$ é dado por uma matriz definida positiva $G_{x}=\left(g_{i j}\right)_{i, j=1}^{n}$, cuja inversa denotaremos por $G_{x}^{-1}=\left(g^{l k}\right)_{l, k=1}^{n}$, então:

$$
(\Delta f) \circ \chi^{-1}:=\left|\operatorname{det} g_{i j}\right|^{-1 / 2} \sum_{k, l=1}^{n} \frac{\partial}{\partial x^{k}}\left(g^{l k}\left|\operatorname{det} g_{i j}\right| \frac{\partial}{\partial x^{l}} \tilde{f}\right) \text {. }
$$

Seguem alguns fatos sobre este operador: ${ }^{1}$

Verifica-se que $1-\Delta: C^{\infty}(\mathcal{M}) \longrightarrow C^{\infty}(\mathcal{M})$ é uma bijeção cuja inversa possui uma extensão $(1-\Delta)^{-1} \in \mathcal{B}\left(L^{2}(\mathcal{M})\right)$, o conjunto dos operadores lineares limitados em $L^{2}(\mathcal{M})$ com a medida induzida da métrica riemanniana $G$. Este operador é positivo e assim existe $\Lambda:=(1-\Delta)^{-1 / 2}$. Verifica-se também que $\Lambda^{-1}\left(C^{\infty}(\mathcal{M})\right)$ é denso em $L^{2}(\mathcal{M})$, e que se $L$ : $C^{\infty}(\mathcal{M}) \longrightarrow C^{\infty}(\mathcal{M})$ é um operador diferencial de primeira ordem, então $(L \circ \Lambda): \Lambda^{-1}\left(C^{\infty}(\mathcal{M})\right) \longrightarrow C^{\infty}(\mathcal{M})$ se estende a um operador limitado em $L^{2}(\mathcal{M})$.

A álgebra de comparação $\mathcal{A}(\mathcal{M})$ é a menor subálgebra de $\mathcal{B}\left(L^{2}(\mathcal{M})\right)$ fechada na topologia da norma e invariante pelo adjunto, contendo:

$$
\left\{M_{a}: a \in C^{\infty}(\mathcal{M}) \text { e } \forall f \in L^{2}(\mathcal{M}), \forall x \in \mathcal{M}, M_{a}(f)(x):=a(x) f(x)\right\} \text { e }
$$

$\left\{L \Lambda: L\right.$ é um operador linear de ordem 1 com coeficientes $\left.C^{\infty}\right\}$.

Prova-se que $\mathcal{A}(\mathcal{M})$ é o fecho da álgebra $\Psi_{c l}^{0}(\mathcal{M})$, o conjunto dos operadores pseudo-diferenciais clássicos de ordem zero, conforme [12], seção 4.

Neste trabalho, consideraremos o caso particular em que $\mathcal{M}$ é a variedade sem bordo compacta $S^{1}$ e estudaremos uma subálgebra $\mathcal{A} \subset \mathcal{B}\left(L^{2}\left(S^{1}\right)\right)$, a qual, como último resultado deste trabalho, demonstraremos ser a álgebra de comparação $\mathcal{A}\left(S^{1}\right)$.

${ }^{1}$ Ver [4]. 
A principal ferramenta usada é o Teorema de Gelfand para $C^{*}$-álgebras comutativas. Veremos que o quociente de $\mathcal{A}$ pelo conjunto dos seus elementos compactos é uma $C^{*}$-álgebra comutativa e daremos uma descrição concreta do seu espaço de Gelfand $M_{\mathcal{A} / \mathcal{K}}$.

Além disso, daremos uma fórmula para o cálculo do índice de um operador de Fredholm em $\mathcal{A}$, a partir da função símbolo $\sigma: M_{n}(\mathcal{A}) \longrightarrow$ $M_{n}\left(C\left(M_{\mathcal{A} / \mathcal{K}}\right)\right)$, que será definida em 9.2 , e provaremos que o índice de um operador diferencial de Fredholm no círculo é sempre nulo.

O índice de Fredholm dos operadores (elípticos) em $\Psi_{c l}^{0}(\mathcal{M})$ foi calculado por Fedosov [6] usando o Teorema de Atiyah-Singer [1]. A fórmula que obteremos na página 46 pode ser vista como um caso particular da fórmula obtida por Fedosov no contexto geral de variedades. 


\section{Algumas Definições E NotaÇÕes}

2.1. Definição. Seja $A$ uma álgebra sobre $\mathbb{C}$ com uma operação $*: A \longrightarrow A$ com as seguintes propriedades: Para todos $a, b \in A$ e todo $\lambda \in \mathbb{C}$ :

(1) $(a+\lambda b)^{*}=a^{*}+\bar{\lambda} b^{*} ;$

(2) $a^{* *}=a$;

(3) $(a b)^{*}=b^{*} a^{*}$.

O par $(A, *)$ é chamado uma álgebra com involução, ou *-álgebra.

2.2. Definição. Uma álgebra de Banach é uma álgebra munida de uma norma $\|\cdot\| \longrightarrow \mathbb{R}_{+}$submultiplicativa; isto é: para todos $a, b \in A,\|a b\| \leq$ $\|a\| \mid\|b\|$, e que é completa nesta norma.

2.3. Definição. Uma $C^{*}$-álgebra é uma álgebra de Banach $A$ com uma involução para a qual vale a seguinte propriedade que a relaciona com a norma: Para todo $a \in A,\left\|a^{*} a\right\|=\|a\|^{2}$.

2.4. Exemplo. Seja $(\mathcal{H},<\cdot, \cdot>)$ um espaço de Hilbert. Pelo teorema de Riesz, sabemos que para todo $T \in \mathcal{B}(\mathcal{H})$ existe $T^{*} \in \mathcal{B}(\mathcal{H})$ tal que, para todos $x, y \in \mathcal{H},\langle T x, y\rangle=<x, T^{*} y>$. Tomando a norma em $\mathcal{B}(\mathcal{H})$ como: $\||| T \mid\|:=\sup \{\|T x\|:\|x\| \leq 1\}$, é simples verificar que a $*$ definida é uma involução que satisfaz as condições acima. $\operatorname{Logo}, \mathcal{B}(\mathcal{H})$ é uma $C^{*}$-álgebra.

2.5. Exemplo. Seja $X$ um espaço Hausdorff localmente compacto, e $C_{0}(X)$ o conjunto das funções complexas que se anulam no infinito. ${ }^{2}$ Se tomarmos a norma do sup; isto é, $\|f\|_{\infty}:=\sup \{|f(x)|: x \in X\}$, e a involução como $f^{*}:=\bar{f}$, então também é fácil verificar que $C_{0}(X)$ é uma $C^{*}$-álgebra.

2.6. Notação. $S^{1}:=\left\{e^{i \theta} \in \mathbb{C}: \theta \in \mathbb{R}\right\}$.

2.7. Definição. Dada $f \in C\left(S^{1}\right)$, definimos $\tilde{f} \in C(\mathbb{R})$ pela regra que, para cada $\theta \in \mathbb{R}$, associa $\tilde{f}(\theta):=f\left(e^{i \theta}\right)$. Diremos que $f$ é diferenciável se, e só se, $\tilde{f}$ é for diferenciável em $\mathbb{R}$. Neste caso definimos $f^{\prime}\left(e^{i \theta}\right):=\frac{d}{d \theta} \tilde{f}(\theta)$. Além disso, denotaremos $f \in C^{\infty}\left(S^{1}\right)$ se, e só se, $\tilde{f} \in C^{\infty}(\mathbb{R})$.

2.8. Definição. Diremos que $f: S^{1} \longrightarrow \mathbb{C}$ é integrável se, e só se, $\tilde{f}$ for integrável em $[-\pi, \pi]$, e denotaremos:

$$
\int_{S^{1}} f d \mu:=\frac{1}{2 \pi} \int_{-\pi}^{\pi} \tilde{f}(\theta) d \theta
$$

\footnotetext{
${ }^{2}$ Ver B.1 para definição.
} 
onde o segundo termo é a integral de Lebesgue em $[-\pi, \pi]$. Mais geralmente, diremos que $f \in L^{p}\left(S^{1}\right)$ se, e só se, $f^{p}$ for integrável.

Além disso, a medida $\mu$ definida acima será chamada de medida de Lebesgue em $S^{1}$.

2.9. Definição. Seja $j \in \mathbb{Z}$. Definimos:

$$
\begin{aligned}
e_{j}: S^{1} & \longrightarrow \mathbb{C} \\
z & \longmapsto e_{j}(z):=z^{j} .
\end{aligned}
$$

Assim, se $z=e^{i \theta}, \tilde{e_{j}}(\theta)=e^{i j \theta}$.

2.10. Teorema (Stone-Weierstrass). Sejam $M$ um espaço métrico compacto $e \mathcal{B} \subset C(M)$ uma subálgebra autoadjunta que contém as constantes e separa pontos de $M$. Então toda $f \in C(M)$ pode ser uniformemente aproximada por funções de $\mathcal{B}$.

Demonstração. Ver pagina 263 de [10].

Os seguintes resultados sobre a densidade das funções $C^{\infty}\left(S^{1}\right)$ em $L^{p}\left(S^{1}\right)$ serão úteis em alguns pontos da dissertação.

2.11. Notação. Seja $\Omega$ um espaço topológico. Denotamos por $C(\Omega)$ o conjunto das funções contínuas de $\Omega$ em $\mathbb{C}$.

2.12. Definição. Sejam $\Omega$ um espaço topológico e $f \in C(\Omega)$. Definimos o suporte de $f$ como o fecho de $\{x \in \mathbb{C}: f(x) \neq 0\}$ em $\mathbb{C}$, e o denotaremos por $\operatorname{supp}(f)$. Denotaremos por $C_{c}(\Omega)$ o sub-conjunto de $C(\Omega)$ cujos elementos têm suporte compacto.

2.13. Teorema. Sejam $p \in[1, \infty), \Omega$ um espaço de medida localmente compacto Hausdorff e $\mu$ uma medida regular em $\Omega$. Então $C_{c}(\Omega)$ é denso em $L^{p}(\Omega)$.

Demonstração. Ver teorema 3.14, e parágrafos anteriores a ele, além da página 41 para a definição de medida regular, em [15].

2.14. Proposição. Se $p \in[1, \infty)$, então $C^{\infty}\left(S^{1}\right)$ é denso em $L^{p}\left(S^{1}\right)$.

Demonstração. Que $C^{\infty}\left(S^{1}\right)$ é $\|\cdot\|_{\infty}$-denso em $C\left(S^{1}\right)$ é uma decorrência direta do Teorema de Stone-Weierstrass 2.10 acima. Como $S^{1}$ tem medida de Lebesgue finita, para todo $p \in[1, \infty),\|\cdot\|_{\infty}$-denso implica $\|\cdot\|_{p}$-denso. Logo, 
$C^{\infty}\left(S^{1}\right)$ é denso em $C\left(S^{1}\right)$ na norma de $L^{p}\left(S^{1}\right)$. Finalmente, pelo teorema acima, tendo em vista que a medida de Lebesgue é uma medida regular, temos que $C_{c}\left(S^{1}\right)$ é denso em $L^{p}\left(S^{1}\right)$. Como $S^{1}$ é compacto $C_{c}\left(S^{1}\right)=C\left(S^{1}\right)$, e segue o resultado.

2.15. Proposição. A família $\left\{e_{j}\right\}_{j \in \mathbb{Z}}$ é ortonormal e completa em $L^{2}\left(S^{1}\right)$.

Demonstração. O espaço vetorial $N$ gerado por $\left\{e_{j}\right\}_{j \in \mathbb{Z}}$ é uma álgebra autoadjunta, que separa pontos e que contém as constantes, pois $1 \equiv e^{i 0 \theta}$. Logo, pelo Teorema de Stone-Weierstrass, $N$ é $\|\cdot\|_{\infty}$-densa em $C\left(S^{1}\right)$. Mas, pelo teorema 2.13, $C\left(S^{1}\right)$ é $\|\cdot\|_{2}$-denso em $L^{2}\left(S^{1}\right)$. Resta então ver que $N$ é $\|\cdot\|_{2}$-densa em $C\left(S^{1}\right)$. Mas isto ocorre, pois se $\left(f_{n}\right)_{n \in \mathbb{N}}$ é uma seqüência em $N$ que converge uniformente para $g \in C\left(S^{1}\right)$, então também converge para $g$ na norma de $L^{2}\left(S^{1}\right)$, pois:

$$
\begin{aligned}
\left\|f_{n}-g\right\|_{2}^{2} & =\int_{S^{1}}\left|f_{n}-g\right|^{2} d \mu \\
& \leq \sup \left\{\left|f_{n}(\theta)-g(\theta)\right|^{2}: \theta \in S^{1}\right\} \\
& =\left\|f_{n}-g\right\|_{\infty}^{2} \stackrel{n \rightarrow \infty}{\longrightarrow} 0 .
\end{aligned}
$$

Segue o resultado.

2.16. Definição. A transformação de Fourier é definida por:

$$
\begin{aligned}
F: L^{2}\left(S^{1}\right) & \longrightarrow l^{2}(\mathbb{Z}) \\
f & \longmapsto F(f):=\left(\int_{S^{1}} \overline{e_{j}} f d \mu\right)_{j \in \mathbb{Z}} .
\end{aligned}
$$

2.17. Notação. Denotarenos, onde for conveniente, $F(f)$ por $\widehat{f}$.

Agora vamos listar algumas propriedades úteis da transformação de Fourier.

2.18. Proposição. A transformação de Fourier é um operador unitário.

Demonstração. De fato, $F$ leva a família ortonormal completa $\left\{e_{j}\right\}_{n \in \mathbb{Z}} \subset$ $L^{2}\left(S^{1}\right)$ na família ortonornal completa $\left\{\left(\delta_{i j}\right)_{j \in \mathbb{Z}}\right\}_{i \in \mathbb{Z}} \subset l^{2}(\mathbb{Z})$.

2.19. Proposição. Seja $f$ derivável em $S^{1}$. Então, $\left({\widehat{f^{\prime}}}_{j}\right)_{j \in \mathbb{Z}}=\left(-i j \widehat{f}_{j}\right)_{j \in \mathbb{Z}}$. 
Demonstração. Fazendo a seguinte integração por partes, obtemos:

$$
\begin{aligned}
-i \widehat{f}_{j}^{\prime} & =\frac{1}{2 \pi} \int_{-\pi}^{\pi} e^{-i j \theta}(-i) \tilde{f}^{\prime}(\theta) d \theta \\
& =\frac{1}{2 \pi}\left\{\left.\left(e^{-i j \theta} \tilde{f}(\theta)\right)\right|_{-\pi} ^{\pi}-\int_{-\pi}^{\pi}-i j e^{-i j \theta}(-i) \tilde{f}(\theta) d \theta\right\} \\
& =j \frac{1}{2 \pi} \int_{-\pi}^{\pi} e^{-i j \theta} \tilde{f}(\theta) d \theta \\
& =j \widehat{f} .
\end{aligned}
$$

Uma importante caracterização das funções em $C^{\infty}\left(S^{1}\right)$ é dada pelo seguinte resultado:

2.20. Proposição. Seja $f \in L^{2}\left(S^{1}\right) . \quad f \in C^{\infty}\left(S^{1}\right)$ se, e só se, para todo $k \in \mathbb{N}, \sup \left\{\left|j^{k} \widehat{f}_{j}\right|: j \in \mathbb{Z}\right\}<\infty$.

Demonstração. Segue da proposição anterior que se $f \in C^{\infty}\left(S^{1}\right)$, então $\widehat{f(k)}_{j}=(-i j)^{k} \widehat{f}_{j}$. Logo,

$$
\left|\widehat{f^{(k)}}{ }_{j}\right|^{2}=\left|j^{k} \widehat{f}_{j}\right|^{2}
$$

Como $f^{(k)} \in C\left(S^{1}\right) \subset L^{2}\left(S^{1}\right)$, temos que $\left(\widehat{f^{(k)}}{ }_{j}\right)_{j \in \mathbb{Z}} \in l^{2}(\mathbb{Z})$. Assim, $\sum_{j \in \mathbb{Z}}\left|j^{k} \widehat{f}_{j}\right|^{2}<\infty$. Portanto, $\sup \left\{\left|j^{k} \widehat{f}_{j}\right|: j \in \mathbb{Z}\right\}<\infty$.

Por outro lado, se $f \in L^{2}\left(S^{1}\right)$ podemos escrever $f$ na base $\left\{e_{j}\right\}_{j \in \mathbb{Z}}$ de $L^{2}\left(S^{1}\right)$; isto é,

$$
f=\sum_{j \in \mathbb{Z}} \widehat{f}_{j} e_{j} .
$$

Vejamos que tal convergência é também uniforme.

Olhando para esta soma como uma função em $\mathbb{R}$ temos:

$$
\tilde{f}=\sum_{j \in \mathbb{Z}} \widehat{f}_{j} \tilde{e}_{j}
$$

Daí, para quase todo $\theta \in \mathbb{R}$, vale:

$$
\tilde{f}(\theta)=\sum_{j \in \mathbb{Z}} \widehat{f_{j}} \tilde{e_{j}}(\theta)=\sum_{j \in \mathbb{Z}} \widehat{f_{j}} e^{i j \theta} .
$$

Como vale a hípótese de que para todo $k \in \mathbb{N}$, $\sup \left\{\left|j^{k} \widehat{f}_{j}\right|: j \in \mathbb{Z}\right\}<\infty$, então, na verdade, para todo $p \in \mathbb{C}[X], \sup \left\{\left|p(j) \widehat{f}_{j}\right|: j \in \mathbb{Z}\right\}<\infty$. Em 
particular, existe $c \in \mathbb{R}$ tal que $\sup \left\{\left|\left(1+j^{2}\right) \widehat{f}_{j}\right|: j \in \mathbb{Z}\right\}<c$. Logo, para cada $j \in \mathbb{Z}$,

$$
\sup \left\{\left|\widehat{f}_{j} e_{j}(\theta)\right|: \theta \in \mathbb{R}\right\}=\left|\widehat{f}_{j}\right|<\frac{c}{1+j^{2}}=: M_{j} .
$$

Como $\sum_{j \in \mathbb{Z}} M_{j}<\infty$, segue, pelo M-teste de Weierstrass, que $\tilde{f}=\sum_{j \in \mathbb{Z}} \widehat{f_{j}} \tilde{e}_{j}$ converge uniformemente. Como cada um dos somandos é uma função contínua, segue que $\tilde{f}$ e conseqüentemente $f$ também o são.

$\mathrm{Na}$ verdade, a série das funções derivadas também converge uniformemente, como vemos por um argumento análogo ao anterior. Considerando que existe $d \in \mathbb{R}$ tal que $\sup \left\{\left|\left(j+j^{3}\right) \widehat{f}_{j}\right|: j \in \mathbb{Z}\right\}<d$, temos:

$$
\sup \left\{\left|\widehat{f}_{j} e_{j}^{\prime}(\theta)\right|: \theta \in \mathbb{R}\right\}=\left|j \widehat{f}_{j}\right|<\frac{d}{1+j^{2}}=: N_{j} .
$$

Assim, segue que $f$ é derivável e $f^{\prime}=\sum_{j \in \mathbb{Z}} \widehat{f}_{j} e_{j}^{\prime}$. Procedendo por indução e usando o polinômio $j^{n}+j^{n+2}$ para mostrar que $f \in C^{n}\left(S^{1}\right)$ e que $f^{(n)}=$ $\sum_{j \in \mathbb{Z}} \widehat{f}_{j} e_{j}^{(n)}$, para todo $n \in \mathbb{N}$, concluímos que $f \in C^{\infty}\left(S^{1}\right)$, como queríamos demonstrar. 


\section{Roteiro da Dissertação}

Sejam $L^{2}\left(S^{1}\right)$ o $\mathbb{C}$-espaço de Hilbert das funções de quadrado integrável no círculo unitário e $\mathcal{B}\left(L^{2}\left(S^{1}\right)\right)$ a $C^{*}$-álgebra dos operadores lineares contínuos em $L^{2}\left(S^{1}\right)$. Vamos considerar duas $C^{*}$-sub-álgebras, $\mathcal{A}_{1}$ e $\mathcal{A}_{2}$, que serão definidas a seguir.

Sejam $a \in C\left(S^{1}\right)$ e $u \in L^{2}\left(S^{1}\right)$. Como $S^{1}$ é compacto, toda função contínua nele definida é limitada. Assim,

$$
\begin{aligned}
\|a u\|_{2}^{2} & =\frac{1}{2 \pi} \int_{-\pi}^{\pi}|\tilde{a}(\theta) \tilde{u}(\theta)|^{2} d \theta \\
& \leq \sup \left\{|\tilde{a}(\theta)|^{2}: \theta \in \mathbb{R}\right\} \frac{1}{2 \pi} \int_{-\pi}^{\pi}|\tilde{u}(\theta)|^{2} d \theta \\
& =\|a\|_{\infty}^{2}\|u\|_{2}^{2}<\infty .
\end{aligned}
$$

Portanto $a u \in L^{2}\left(S^{1}\right)$.

3.1. Definição. Seja $a \in C\left(S^{1}\right)$. Definimos então o seguinte operador:

$$
\begin{aligned}
M_{a}: L^{2}\left(S^{1}\right) & \longrightarrow L^{2}\left(S^{1}\right) \\
u & \longmapsto M_{a}(u),
\end{aligned}
$$

onde $M_{a}(u)(x):=a(x) u(x)$, para todo $x \in S^{1}$.

3.2. Observação. É claro que nesta definição estamos escrevendo $u$ por $[u]$ e $M_{a}(u)$ por $[a u]$ e, embora daqui para frente esta simplificação seja adotada, é necessário notar antes que esta função está bem definida pois se $u=v \mathrm{em}$ quase todo ponto, então é claro que $a u=a v$ em quase todo ponto.

3.3. Notação. $\mathcal{A}_{1}:=\left\{M_{a}: a \in C\left(S^{1}\right)\right\}$

\subsection{Definição.}

$$
\begin{aligned}
\mathcal{M}: C\left(S^{1}\right) & \longrightarrow \mathcal{A}_{1} \\
a & \longmapsto M_{a} .
\end{aligned}
$$

3.5. Proposição. $\mathcal{A}_{1}$ é uma $C^{*}$-subálgebra de $\mathcal{B}\left(L^{2}\left(S^{1}\right)\right)$.

Demonstração. É claro que $I d=M_{1} \in \mathcal{A}_{1}$ e que $\mathcal{A}_{1}$ é uma sub-álgebra da álgebra dos operadores lineares em $L^{2}\left(S^{1}\right)$, com as operações nela definidas. Para ver que $\mathcal{A}_{1} \subset B\left(L^{2}\left(S^{1}\right)\right)$ - ou seja, que $M_{a}$ é limitado, se $a \in C\left(S^{1}\right)$ basta observar que $\left\|M_{a}\right\| \leq\|a\|_{\infty}$. 
De fato, como $\left\|M_{a}\right\|=\sup \left\{\left\|M_{a}(u)\right\|_{2}: u \in L^{2}\left(S^{1}\right)\right.$ e $\left.\|u\|_{2} \leq 1\right\}$, tomando $\|u\|_{2} \leq 1$, temos que:

$$
\begin{aligned}
\left(\int_{S^{1}}|a u|^{2} d \mu\right)^{1 / 2} & \leq\left(\max \left\{|a(z)|^{2}: z \in S^{1}\right\}\right)^{1 / 2}\left(\int_{S^{1}}|u|^{2} d \mu\right)^{1 / 2} \\
& =\|a\|_{\infty}\|u\|_{2} \leq\|a\|_{\infty}
\end{aligned}
$$

Ou seja, $\left\|M_{a}\right\| \leq\|a\|_{\infty}$. Portanto, $\mathcal{A}_{1}$ é uma sub-álgebra de $B\left(L^{2}\left(S^{1}\right)\right)$.

Precisamos verificar que a involução é fechada para elementos de $\mathcal{A}_{1}$ :

De fato, seja $a \in C\left(S^{1}\right)$. Então, para todos $u, v \in L^{2}\left(S^{1}\right)$ :

$$
\begin{aligned}
<M_{a}^{*}(u), v> & =<u, M_{a}(v)>=\int_{S^{1}} u(\overline{a v}) d \mu \\
& =\int_{S^{1}}(\bar{a} u)(\bar{v}) d \mu=<M_{\bar{a}}(u), v>.
\end{aligned}
$$

Logo, $M_{a}^{*}=M_{\bar{a}} \in \mathcal{A}_{1}$, pois $\bar{a} \in C\left(S^{1}\right)$.

Finalmente, resta ver que $\mathcal{A}_{1}$ é fechada. Para tanto, basta observar que, da continuidade de $a$, existe $z_{0} \in S^{1}$ tal que $\left|a\left(z_{0}\right)\right|=\|a\|_{\infty}$, e que para todo $\varepsilon>0$ existe $V$ vizinhança de $z_{0}$ em $S^{1}$ tal que, se $z \in V$, então $|a(z)|>\|a\|_{\infty}-\varepsilon$. Tome $\phi:=\frac{1}{\mu(V)} \chi_{V}$. Assim, $\|\phi\|_{2}=1, \operatorname{supp}(\phi) \subset V \mathrm{e}$

$$
\left\|M_{a}(\phi)\right\|_{2}^{2}=\int_{S^{1}}|a|^{2}|\phi|^{2} d \mu \geq\left(\|a\|_{\infty}-\varepsilon\right)^{2}\|\phi\|_{2}^{2}
$$

Como $\varepsilon$ é arbitrário, $\left\|M_{a}\right\| \geq\|a\|_{\infty}$.

Logo, a aplicação $\mathcal{M}$ é uma isometria, pois já vimos que $\left\|M_{a}\right\| \leq\|a\|_{\infty}$. Como $\left(C\left(S^{1}\right),\|\cdot\|_{\infty}\right)$ é completo, segue a tese.

3.6. Corolário. $\mathcal{M}$ é uma isometria.

3.7. Definição. Seja $\mathbb{Z}$ o conjunto dos números inteiros. Tomando dois pontos fora de $\mathbb{Z}$, que denotaremos por: $-\infty$ e $+\infty$, declaramos $X:=\mathbb{Z} \cup$ $\{-\infty,+\infty\}$. Em $X$ definimos uma topologia através de vizinhanças básicas de seus pontos: Se $p \in \mathbb{Z} \subset X$, então todo subconjunto de $X$ ao qual $p$ pertença é uma vizinhança de $p$. Se $p=-\infty$, então todo subconjunto de $X$ ao qual $p$ pertença e que contenha um segmento inicial de $\mathbb{Z}$ é uma vizinhança de $p$. Se $p=+\infty$, então é só trocar inicial por final na frase acima.

3.8. Observação. Com esta topologia, é fácil verificar que $X$ é um espaço topológico compacto que contém $\mathbb{Z}$ com a topologia discreta como subespaço 
denso. Além disso, também é imediato ver que $C(X)$ é isomorfo a:

$$
\left\{b \in C(\mathbb{Z}): \text { existem } \lim _{j \rightarrow+\infty} b(j) \text { e } \lim _{j \rightarrow-\infty} b(j)\right\} .
$$

3.9. Definição. Seja $b=\left(b_{n}\right)_{n \in \mathbb{Z}} \in C(X)$. Definimos o seguinte operador:

$$
\begin{aligned}
M_{b}: l^{2}(\mathbb{Z}) & \longrightarrow l^{2}(\mathbb{Z}) \\
u & \longmapsto M_{b}(u),
\end{aligned}
$$

onde $u=\left(u_{n}\right)_{n \in \mathbb{Z}}$ e $M_{b}(u):=\left(b_{n} u_{n}\right)_{n \in \mathbb{Z}}$.

Note que $M_{b}$ agora denota um operador de $l^{2}(\mathbb{Z})$ em si mesmo (e não de $L^{2}\left(S^{1}\right)$ em si mesmo). O uso em contexto deve deixar claro de que operador estamos falando.

3.10. Definição. Seja $b \in C(X)$. Definimos o seguinte operador:

$$
\begin{aligned}
D_{b}: L^{2}\left(S^{1}\right) & \longrightarrow L^{2}\left(S^{1}\right) \\
v & \longmapsto D_{b}(v),
\end{aligned}
$$

onde $D_{b}(v):=\left(F^{-1} \circ M_{b} \circ F\right)(v)$.

3.11. Observação. É necessário tomar o cuidado de apontar a simplificação da notação acima. De fato, onde se escreve $v$, entenda-se $[v]$, e $D_{b}(v)$ deve ser entendido por $\left[\left(F^{-1} M_{b} F\right)([v])\right]$.

3.12. Notação. $\mathcal{A}_{2}:=\left\{D_{b}: b \in C(X)\right\}$

\subsection{Definição.}

$$
\begin{aligned}
\mathcal{D}: C(X) & \longrightarrow \mathcal{A}_{2} \\
b & \longmapsto D_{b} .
\end{aligned}
$$

3.14. Proposição. $\mathcal{A}_{2}$ é uma $C^{*}$-subálgebra.

Demonstração. A idéia é análoga à da demonstração de que $\mathcal{A}_{1}$ é $C^{*}$-álgebra, observando-se a diferença que, como a transformação de Fourier é um operador unitário, $\left\|D_{b}\right\|=\left\|M_{b}\right\|$, e, além disso, $\left\|M_{b}\right\|=\|b\|_{\infty}$. Isto porque, por um lado, se $c \in l^{2}(\mathbb{Z})$, então:

$$
\left\|M_{b}(c)\right\|_{2}^{2}=\left\|\left(b_{j} c_{j}\right)_{j \in \mathbb{Z}}\right\|_{2}^{2} \leq\|b\|_{\infty}^{2}\|c\|_{2}^{2}
$$

logo, $\left\|M_{b}\right\| \leq\|b\|_{\infty}$. 
Por outro lado, se existe $n \in \mathbb{Z}$ tal que: $\left|b_{n}\right|=\|b\|_{\infty}$, então $\left\|M_{b}\left(e_{n}\right)\right\|_{2}=$ $\left|b_{n}\right|=\|b\|_{\infty}\left(\operatorname{com} e_{n}:=\left(\delta_{j n}\right)_{j \in \mathbb{Z}}\right)$ e assim $\left\|M_{b}\right\| \geq\|b\|_{\infty}$. Se não existe tal $n \in \mathbb{Z}$, então podemos assumir, sem perda de generalidade, que:

$$
\lim _{j \rightarrow+\infty} b(j)=\|b\|_{\infty}
$$

Neste caso, dado $\varepsilon>0$, existe $m \in \mathbb{Z}$ tal que se $k>m$, então: $\left|b_{k}\right|>$ $\|b\|_{\infty}-\varepsilon$. Assim, $\left\|M_{b}\left(e_{k}\right)\right\|_{2}=\left|b_{k}\right|>\|b\|_{\infty}-\varepsilon$. Logo, para todo $\varepsilon>$ $0,\left\|M_{b}\right\| \geq\|b\|_{\infty}-\varepsilon$. Assim, $\left\|M_{b}\right\|=\|b\|_{\infty}$, como queríamos. Segue o resultado.

3.15. Corolário. $\mathcal{D}$ é uma isometria.

3.16. Definição. Seja $T$ uma transformação linear entre espaços de Banach $\mathcal{X}$ e $\mathcal{Y}$. Dizemos que $T$ é uma transformação compacta se, e só se, para todo $A \subset \mathcal{X}$ limitado, $\overline{T(A)}$ é compacto em $\mathcal{Y}$.

3.17. Notação. $\mathcal{K}:=\left\{T \in \mathcal{B}\left(L^{2}\left(S^{1}\right)\right): T\right.$ é um operador compacto $\}$.

3.18. Definição. Sejam $A$ e $B$ operadores em um espaço vetorial $X$. Definimos o comutador de $A$ e $B$ como $A B-B A$ e o denotamos por $[A, B]$.

3.19. Definição. Denotaremos por $\mathcal{A}$ a álgebra gerada por $\mathcal{A}_{1}$ e $\mathcal{A}_{2}$.

Nossos objetivos serão:

(1) Mostrar que se $A, B \in \mathcal{A}$, então $[A, B] \in \mathcal{K}$;

(2) Mostrar que $\mathcal{K} \subset \mathcal{A}$;

(3) Mostrar que $\mathcal{A} / \mathcal{K}$ é uma $C^{*}$-álgebra comutativa;

(4) Dar uma descrição concreta do espaço de Gelfand de $\mathcal{A} / \mathcal{K}$ e mostrar que ele é homeomorfo a uma reunião disjunta de duas cópias de $S^{1}$. Vamos definir e usar a função símbolo de um operador em $\mathcal{A}$;

(5) Dar uma fórmula para o cálculo do índice de um operador de Fredholm de $\mathcal{A}$, a partir do conhecimento da função símbolo do operador;

(6) Demonstrar um critério para decidir se um operador em $M_{n}(\mathcal{A})$ é $d e$ Fredholm. Para tanto vamos definir e usar a função símbolo de um operador em $M_{n}(\mathcal{A})$;

(7) Generalizar a fórmula do indice de um operador de Fredholm para $M_{n}(\mathcal{A}) ; \mathrm{e}$

(8) Mostrar uma aplicação para operadores diferenciais. 


\section{Os Comutadores de $\mathcal{A}$ são Compactos}

Vamos mostrar que $[A, B]$ é compacto, sempre que $A, B \in \mathcal{A}$. Por uma questão de fluência, alguns resultados utilizados serão provados separadamente no apêndice.

Vamos supor inicialmente que $A=M_{a}$ e que $B=D_{e_{i}}$, com $e_{i}=\left(\delta_{i j}\right)_{j \in \mathbb{Z}}$ e $i \in \mathbb{Z}$. Neste caso temos que:

$$
\begin{aligned}
{[A, B] } & =A B-B A \\
& =M_{a} D_{e_{i}}-D_{e_{i}} M_{a} \\
& =M_{a} F^{-1} M_{e_{i}} F-F^{-1} M_{e_{i}} F M_{a}
\end{aligned}
$$

tem posto finito, já que a imagem de $M_{e_{i}}$ está contida em $\mathbb{C} e_{i}$ e os operadores de posto finito formam um ideal bilateral em $\mathcal{B}\left(L^{2}\left(S^{1}\right)\right)$, por 6.3. Logo, como todo operador de posto finito é compacto, $M_{a} D_{e_{i}}-D_{e_{i}} M_{a}$ é compacto.

Agora, suponhamos que $A=M_{a}$ e que $B=D_{b}, \operatorname{com} b(-\infty)=b(+\infty)=0$ e vejamos que o resultado vale.

De fato, se considerarmos inicialmente para cada $k \in \mathbb{Z}$ a função:

$$
\begin{aligned}
b^{k}: X & \longrightarrow \mathbb{C} \\
n & \longmapsto b_{n}^{k},
\end{aligned}
$$

onde

$$
b^{k}:=\left(b_{n}^{k}\right):=\left\{\begin{aligned}
b_{n}, & \text { se }|n| \leq k \\
0, & \text { se }|n|>k,
\end{aligned}\right.
$$

teremos que $D_{b^{k}}=\sum_{i=-k}^{k} b_{i} D_{e_{i}}$ e portanto tem posto finito. Mas então:

$$
\begin{aligned}
\left\|D_{b}-D_{b^{k}}\right\| & =\left\|F^{-1}\left(M_{b}-M_{b^{k}}\right) F\right\| \\
& \leq\left\|F ^ { - 1 } \left|\left\||| M_{b}-M_{b^{k}}|\||| F\|\right.\right.\right. \\
& =\left\|M_{b}-M_{b^{k}}\right\| \\
& =\sup _{n>k}\left|b_{n}-b_{n}^{k}\right| \stackrel{k \rightarrow \infty}{\longrightarrow} 0
\end{aligned}
$$

O que quer dizer que $D_{b}$ é limite de operadores de posto finito e portanto é compacto, por 6.4.

Agora, como:

$$
\left\|M_{a} D_{b}-D_{b} M_{a}-\left(M_{a} D_{b^{k}}-D_{b^{k}} M_{a}\right)\right\| \leq 2\left\|M_{a}\right\|\left\|D_{b}-D_{b^{k}}\right\| \stackrel{k \rightarrow \infty}{\longrightarrow} 0
$$

temos que $\left[M_{a}, D_{b}\right]$ é compacto, pois é limite de operadores compactos. 
4.1. Notação. Agora, precisaremos definir a seguinte função:

$$
\begin{aligned}
\operatorname{sgn}: \mathbb{Z} & \longrightarrow \mathbb{C} \\
m & \longmapsto \operatorname{sgn}(m):=\left\{\begin{array}{rr}
-1, & \text { se } m<0 \\
0, & \text { se } m=0 \\
1, & \text { se } m>0
\end{array}\right.
\end{aligned}
$$

Para ver que $\left[M_{a}, D_{b}\right]$ é compacto com $a \in C\left(S^{1}\right)$ e $b \in C(X)$ vamos usar o Teorema de Stone-Weierstrass e o seguinte importante lema.

4.2. Lema. Se $a \in C^{\infty}\left(S^{1}\right)$, então $\left[M_{a}, D_{\text {sgn }}\right]$ é compacto.

Demonstração. Veja apêncice A.

Seja agora $b \in C(X)$. Vamos mostrar que existem $A, B \in \mathbb{C}$ e $b_{0} \in C_{0}(X)$, visto como subespaço de $C(\mathbb{Z})$, tais que $b=A s g n+B 1+b_{0}$.

De fato, basta que $A$ e $B$ satisfaçam $A+B=b(+\infty)$ e $B-A=b(-\infty)$. Ou seja, é só tomar $A=\frac{b(+\infty)-b(-\infty)}{2}$ e $B=\frac{b(+\infty)+b(-\infty)}{2}$ e verificar que $b_{0}(+\infty)=b_{0}(-\infty)=0$.

Então, se $a \in C^{\infty}\left(S^{1}\right)$ e $b \in C(X)$ :

$$
\left[M_{a}, D_{b}\right]=\left[M_{a}, D_{A s g n+B 1+b_{0}}\right]=A\left[M_{a}, D_{s g n}\right]+B\left[M_{a}, D_{1}\right]+\left[M_{a}, D_{b_{0}}\right]
$$

onde a primeira parcela é um operador compacto, pelo lema; a segunda é 0 , pois $D_{b_{0}}=I d$ e portanto comuta com qualquer elemento; e a terceira é um operador compacto pelos casos anteriores. Logo, neste caso, $\left[M_{a}, D_{b}\right]$ é um operador compacto.

Então, pelo Teorema de Stone-Weierstrass, se $a \in C\left(S^{1}\right)$ existe uma seqüência de funções $\left(f_{n}\right)_{n \in \mathbb{N}}$ em $C^{\infty}\left(S^{1}\right)$ que converge uniformemente para $a$. Tomando $b \in C(X)$, segue que:

$$
\begin{aligned}
\left\|\left[M_{f_{n}}, D_{b}\right]-\left[M_{a}, D_{b}\right]\right\| & \leq 2\left\|D _ { b } \left|\left\||| M_{f_{n}}-M_{a}\right\|\right.\right. \\
& =2\|b\|\left\|\mid f_{n}-a\right\| \stackrel{n \rightarrow \infty}{\longrightarrow} 0
\end{aligned}
$$

Como cada $\left[M_{f_{n}}, D_{b}\right]$ é compacto, segue que $\left[M_{a}, D_{b}\right]$ também o é, pois $\mathcal{K}$ é fechado. Logo, o resultado vale para $(a, b) \in C\left(S^{1}\right) \times C(X)$.

Provemos agora que $[A, B]$ é compacto, se $A, B \in \mathcal{A}_{f}$, a álgebra finitamente gerada por $\mathcal{A}_{1}$ e $\mathcal{A}_{2}$.

Para tanto, basta ver que $\left[\prod_{i=1}^{n} A_{i} B_{i}, \prod_{j=1}^{m} C_{j} D_{j}\right]$ é compacto para quaisquer $A_{i}, C_{j} \in \mathcal{A}_{1}$ e $B_{i}, D_{j} \in \mathcal{A}_{2}$, pois o comutador é bilinear e todo elemento de $\mathcal{A}_{f}$ é uma combinação linear destes produtos. 
4.3. Proposição. $\left[\prod_{i=1}^{n} A_{i} B_{i}, \prod_{j=1}^{m} C_{j} D_{j}\right]$ é um operador compacto para quaisquer $A_{i}, C_{j} \in \mathcal{A}_{1}$ e $B_{i}, D_{j} \in \mathcal{A}_{2}$.

Demonstração. Vamos mostrar este fato por indução em $m+n$.

Se $m+n=0$, então $m=n=0$ e

$$
\left[\prod_{i=1}^{n} A_{i} B_{i}, \prod_{j=1}^{m} C_{j} D_{j}\right]=[I d, I d]=0
$$

que é compacto.

Suponhamos agora que o resultado vale para $m+n$ e vejamos que vale para $m+n+1$. De fato, se $k+l=n+m+1$, então, pela observação algébrica de que:

$$
\begin{aligned}
{[A B, C] } & =A B C-C A B \\
& =A B C-A C B+A C B-C A B \\
& =A[B, C]+[A, C] B,
\end{aligned}
$$

segue que:

$$
\left[\prod_{i=1}^{k} A_{i} B_{i}, \prod_{j=1}^{l} C_{j} D_{j}\right]=A_{1} B_{1}\left[\prod_{i=2}^{k} A_{i} B_{i}, \prod_{j=1}^{l} C_{j} D_{j}\right]+\left[A_{1} B_{1}, \prod_{j=1}^{l} C_{j} D_{j}\right] \prod_{i=2}^{k} A_{i} B_{i} .
$$

Mas para os dois comutadores que aparecem no membro direito vale a hipótese de indução e portanto, como os operadores compactos formam um ideal bilateral em $\mathcal{B}\left(L^{2}\left(S^{1}\right)\right.$ ) (vide seção 6), segue a tese.

Então já sabemos que $[A, B]$ é compacto, se $A, B \in \mathcal{A}_{f}$. Mas, se $A \in$ $\mathcal{A}_{f}$ e $B \in \mathcal{A}$, existe uma seqüência de elementos de $\mathcal{A}_{f},\left(B_{n}\right)_{n \in \mathbb{N}}$, tal que $\left\|B-B_{n}\right\| \stackrel{n \rightarrow \infty}{\longrightarrow} 0$.

Logo,

$$
\begin{aligned}
\left\|[A, B]-\left[A, B_{n}\right]\right\| & =\left\|A\left(B-B_{n}\right)-\left(B-B_{n}\right) A\right\| \\
& \leq 2\|A\|\left\|\left(B-B_{n}\right)\right\| \stackrel{n \rightarrow \infty}{\longrightarrow} 0 .
\end{aligned}
$$

Assim, $[A, B]$ é limite de operadores compactos e portanto é compacto.

Por um raciocínio análogo, temos que $[A, B]$ é compacto, se $A, B \in \mathcal{A}$, como queríamos demostrar. 


\section{Os Operadores Compactos estão em $\mathcal{A}$}

Seja $\mathcal{K}$ o conjunto dos operadores compactos em $\mathcal{B}\left(L^{2}\left(S^{1}\right)\right)$. O objetivo desta seção é mostrar que $\mathcal{K} \subset \mathcal{A}$. A idéia é utilizar o teorema enunciado abaixo, cuja demonstração se encontra em [5], na página 126:

5.1. Teorema. Sejam $\mathcal{H}$ um espaço de Hilbert, $\mathcal{B}$ uma $C^{*}$-álgebra irredutivel contida em $B(\mathcal{H})$ e $\mathcal{K}$ o conjunto dos operadores compactos em $B(\mathcal{H})$. Se $\mathcal{B} \cap \mathcal{K} \neq\{0\}$, então $\mathcal{K} \subset \mathcal{B}$.

Vamos, portanto, mostrar que a nossa $C^{*}$-sub-álgebra $\mathcal{A}$ é irredutível. Para isso, é claro, precisamos primeiro entender o que é uma $C^{*}$-sub-álgebra ser irredutível.

5.2. Definição. Seja $T$ um operador em um espaço de Hilbert $\mathcal{H}$ e $\mathcal{F}$ um subespaço fechado de $\mathcal{H}$. Dizemos que $\mathcal{F}$ é redutivel por $T$ se, e só se, $T(\mathcal{F}) \subset \mathcal{F}$ e $T\left(\mathcal{F}^{\perp}\right) \subset \mathcal{F}^{\perp}$.

5.3. Definição. Seja $\mathcal{S}$ um subconjunto de $\mathcal{B}(\mathcal{H})$. Dizemos que $\mathcal{S}$ é irredutível se, e só se, não existe um subespaço fechado próprio de $\mathcal{H}$ que seja redutível para todo $T \in \mathcal{S}$.

Com isto em vista, nosso trabalho daqui para frente será mostrar que $\mathcal{A}$ é irredutível. O seguinte lema nos fornece uma maneira para verificarmos este fato.

5.4. Lema. Se $H$ é um espaço de Hilbert e $S \subset B(H)$ é tal que para todo $x \in H$ não nulo $S x=\{T x: T \in S\}$ é denso em $H$, então $S$ é irredutível.

Demonstração. Se $S$ fosse redutível, então $H$ seria a soma direta de dois subespaços ortogonais, fechados e não vazios $H_{1}$ e $H_{2}$, ambos $T$-invariantes, para toda $T \in S$. Assim, se $y \in H_{2}$ é não nulo, $d\left(y, H_{1}\right)=\|y\|>0$. Mas, fixado $x \in H_{1}$ não nulo, temos que $T(x) \in H_{1}$, para toda $T \in S$; ou seja, $S x$ não seria denso em $H$.

O que faremos a seguir é mostrar que $\mathcal{A}$ satisfaz a hipótese do lema ${ }^{3}$. Ou seja, mostraremos que para toda $f \in L^{2}\left(S^{1}\right)$ e toda $\phi \in C^{\infty}\left(S^{1}\right)$ existe

\footnotetext{
${ }^{3} \mathrm{~A}$ demonstração apresentada aqui é uma adaptação daquela feita para o lema V.I.I de [4], que afirma que toda álgebra de comparação sobre uma variedade de dimensão maior ou igual que 2 contém o ideal dos compactos.
} 
$A \in \mathcal{A}$ tal que $A f=\phi$, o que é suficiente, já que $C^{\infty}\left(S^{1}\right)$ é denso em $L^{2}\left(S^{1}\right)$, pela proposição 2.14 acima.

5.5. Definição. Consideremos a seqüência:

$$
\begin{aligned}
b: \mathbb{Z} & \longrightarrow \mathbb{C} \\
j & \longmapsto b(j):=\frac{1}{1+(2 \pi j)^{2}} .
\end{aligned}
$$

e o respectivo operador em $\mathcal{A}_{2}, D_{b}: \mathbb{Z} \longrightarrow \mathbb{C}$.

5.6. Proposição. Seja $g \in C^{\infty}\left(S^{1}\right)$. Calcular $D_{b}(g)$ é equivalente a encontrar $v \in C^{\infty}\left(S^{1}\right)$ tal que: $v-4 \pi^{2} v^{\prime \prime}=g$.

Demonstração. Pela proposição 2.19,

$$
\begin{aligned}
v-4 \pi^{2} v^{\prime \prime}=g & \Leftrightarrow \forall j \in \mathbb{Z}:\left(1+4 \pi^{2} j^{2}\right) \widehat{v}_{j}=\widehat{g}_{j} \\
& \Leftrightarrow \forall j \in \mathbb{Z}: \widehat{v}_{j}=\frac{\widehat{g}_{j}}{\left(1+4 \pi^{2} j^{2}\right)} \Leftrightarrow v=D_{b}(g) .
\end{aligned}
$$

Portanto $v(g)=D_{b}(g)$, para toda $g \in C^{\infty}\left(S^{1}\right)$.

Agora, vamos passar a obter informações de $D_{b}$ resolvendo diretamente a equação diferencial acima. Para isto, vamos resolver o seguinte problema de valor de contorno, cuja resolução detalhada se encontra no apêndice C:

$$
\left\{\begin{array}{l}
\tilde{v}-4 \pi^{2} \tilde{v}^{\prime \prime}=\tilde{g} \\
\tilde{v}(-\pi)=\tilde{v}(\pi) \\
\tilde{v}^{\prime}(-\pi)=\tilde{v}^{\prime}(\pi),
\end{array}\right.
$$

onde $\tilde{g} \in C^{\infty}([-\pi, \pi])$ e $\tilde{g}(-\pi)=\tilde{g}(\pi)$. Das contas feitas no apêndice, obtemos que:

$$
v(z)=V(g)(z):=\int_{S^{1}} H(z, w) g(w) d w,
$$

onde o núcleo $H \in L^{2}\left(S^{1} \times S^{1}\right)$ é um núcleo positivo. Mas a regra de associação acima também faz sentido para elementos em $L^{2}\left(S^{1}\right)$, e não apenas em $C^{\infty}\left(S^{1}\right)$. Podemos então ver $V$ como um operador de $L^{2}\left(S^{1}\right)$. Já sabemos que $D_{b}$ e $V$ coincidem e $C^{\infty}\left(S^{1}\right)$, que é denso em $L^{2}\left(S^{1}\right)$. Além disso, como $D_{b}$ e $V$ são contínuos em $L^{2}\left(S^{1}\right)$ (o primeiro é contínuo, pela demonstração da proposição 3.14 , e o segundo é contínuo pelo lema A.9), então segue que $V=D_{b}$. Disso temos o seguinte:

5.7. Corolário. $D_{b}$ é um operador integral com núcleo contínuo positivo. 
5.8. Proposição. $\operatorname{Im}\left(D_{b}\right) \subset C\left(S^{1}\right)$.

Demonstração. Seja $z_{n} \stackrel{n \rightarrow \infty}{\longrightarrow} z$ uma seqüência convergente em $S^{1}$. Como $H$ é contínua, definindo $H_{n} \in L^{2}\left(S^{1}\right)$ pela regra de associação $H_{n}(w):=$ $H\left(z_{n}, w\right)$, temos que:

$$
\sup \left\{\left|H\left(z_{n}, w\right)\right|: n \in \mathbb{N} \text { e } w \in S^{1}\right\} \leq \sup \left\{|H(z, w)|: S^{1} \times S^{1}\right\}=: M<\infty
$$

Ou seja, para todo $w \in S^{1}$ :

$$
\left|H(z, w)-H_{n}(w)\right| \leq 2 M .
$$

Seja $g \in L^{2}\left(S^{1}\right)$. Então $\left|\left\{H(z, w)-H\left(z_{n}, w\right)\right\} g(w)\right|$ é limitada por $2 M|g|$, que é integrável, pois $L^{2} \subset L^{1}$, já que $S^{1}$ tem medida finita. Assim,

$$
\left|D_{b}(g)(z)-D_{b}(g)\left(z_{n}\right)\right| \leq \int_{S^{1}}\left|\left(H(z, w)-H\left(z_{n}, w\right)\right) g(w)\right| d w
$$

pela desigualdade de Hölder. Logo, pelo Teorema da Convergência Dominada, segue que:

$$
\left|D_{b}(g)(z)-D_{b}(g)\left(z_{n}\right)\right| \stackrel{n \rightarrow \infty}{\longrightarrow} 0
$$

Logo, $V(g) \in C\left(S^{1}\right)$, como queríamos demonstrar.

Portanto, já sabemos que a imagem de $D_{b} \in \mathcal{B}\left(L^{2}\left(S^{1}\right)\right)$ está contida em $C\left(S^{1}\right)$. Isto faz com que agora sejamos capazes de mostrar que para toda $f \in L^{2}\left(S^{1}\right)$ e toda $\phi \in C^{\infty}\left(S^{1}\right)$ existe $A \in \mathcal{A}$ tal que $A f=\phi$. O que faremos é construir uma tal $A \in \mathcal{A}$ que faça este serviço.

5.9. Notação. Denotaremos a parte real de um número complexo $z$ por $\operatorname{Re}(z)$.

5.10. Proposição. Se $f \in L^{2}\left(S^{1}\right)$ é não nula $\mu$-q.s., então $\mathcal{A} f$ é denso em $L^{2}\left(S^{1}\right)$.

Demonstração. Vejamos que para toda $f \in L^{2}\left(S^{1}\right)$ não nula $\mu$-q.s. e toda $\phi \in C^{\infty}\left(S^{1}\right)$ existe $A \in \mathcal{A}$ tal que $A f=\phi$.

Como ker $M_{b}=\{0\}$, então $M_{b}$ é injetora, e como $D_{b}=F^{-1} M_{b} F$ e $F$ é isomorfismo, segue que $D_{b}$ é injetora. Portanto, fixada $f \in L^{2}\left(S^{1}\right)$ não nula $\mu$-q.s., temos que $D_{b}(f) \neq 0$. Logo, existe $z_{0} \in S^{1}$ tal que $D_{b}(f)\left(z_{0}\right) \neq 0$.

Se $D_{b}(f)\left(z_{0}\right)=\rho e^{i \theta}$, é só tomar $D:=e^{-i \theta} D_{b}$, e teremos então que:

$$
D(f)\left(z_{0}\right)=e^{-i \theta} D_{b}(f)\left(z_{0}\right)=e^{-i \theta} \rho e^{i \theta}=\rho>0 .
$$


Então, pela proposição 5.8 acima, existe $U$ vizinhança de $z_{0} \mathrm{em} S^{1}$ tal que $\operatorname{Re}(D(f))(w)>0$, para todo $w \in U$.

Seja $g \in C^{\infty}\left(S^{1}\right)$ tal que $g\left(z_{0}\right)=1, g \geq 0$ e $\operatorname{supp}(g) \subset U$. Definindo $h:=g D(f)$, temos que $\operatorname{Re}(h) \geq 0$ em $S^{1}$ e $\operatorname{Re}(h)\left(z_{0}\right)>0$. Assim, temos que $D_{b}(h)(z) \neq 0$ para todo $z \in S^{1}$, pois:

$$
\operatorname{Re}\left(D_{b}(h)(z)\right)=\int_{-\pi}^{\pi} H(z, w) \operatorname{Re}(h)(w) d w>0,
$$

para todo $z \in S^{1}$. Isto porque este integrando é uma função não negativa, que é estritamente positiva no aberto $U$ que tem medida positiva.

Tomando $l:=D_{b}(h)$, vemos que esta é uma função contínua que não se anula em $S^{1}$ (já que a sua parte real é sempre positiva), logo inversível. Assim,

$$
\phi=\frac{\phi l}{l}=\frac{\phi}{l} D_{b}(h)=\frac{\phi}{l} D_{b}(g D(f))=\left(M_{\phi / l} D_{b} M_{e^{-i \theta} g} D_{b}\right)(f) ;
$$

ou seja, tomando $A:=M_{\phi / l} D_{b} M_{e^{-i \theta} g} D_{b}$, segue que $A \in \mathcal{A}$ e $A f=\phi$. Como por $2.14 C^{\infty}\left(S^{1}\right)$ é denso em $L^{2}\left(S^{1}\right)$, segue o resultado.

Logo, $\mathcal{A}$ é irredutível em $\mathcal{B}\left(L^{2}\left(S^{1}\right)\right)$, como queríamos demonstrar.

Além disso, $\mathcal{A} \cap \mathcal{K} \neq\{0\}$, pois $D_{e_{1}} \in \mathcal{A} \cap \mathcal{K}$. Logo, pelo teorema 5.1, provamos que $\mathcal{K} \subset \mathcal{A}$. 


\section{6. $\mathcal{A} / \mathcal{K}$ é uma $C^{*}$-Álgebra Comutativa}

Seja $\mathcal{K}$ o conjunto dos operadores compactos em $\mathcal{B}\left(L^{2}\left(S^{1}\right)\right)$. Como vimos, $\mathcal{K} \subset \mathcal{A}$. O objetivo desta seção é mostrar que $\mathcal{A} / \mathcal{K}$ é também uma $C^{*}$ álgebra comutativa. Para tanto, vamos utilizar o teorema enunciado abaixo, cuja demonstração se encontra em [5], na página 124:

6.1. Teorema. Se $\mathcal{U}$ é uma $C^{*}$-álgebra e $\mathcal{I}$ é um ideal bilateral fechado em $\mathcal{U}$, então $\mathcal{I}$ é auto-adjunto e a álgebra quociente $\mathcal{U} / \mathcal{I}$ é uma $C^{*}$-álgebra com respeito à involução induzida pela projeção canônica.

Assim, precisamos verificar que $\mathcal{K}$ é um ideal bilateral fechado de $\mathcal{A}$.

Para ver que $\mathcal{K}$ é um ideal à esquerda, vamos usar a seguinte caracterização de transformações lineares compactas entre espaços normados.

6.2. Lema. Sejam $X$ e $Y$ espaços de Banach e $T \in B(X, Y)$. Então $T$ é compacto se, e só se, para toda seqüência limitada $\left(x_{n}\right)_{n \in \mathbb{N}}$ em $X,\left(T x_{n}\right)_{n \in \mathbb{N}}$ possui subseqüência convergente em $Y$.

Demonstração. Se $T$ é compacto e $\left(x_{n}\right)_{n \in \mathbb{N}}$ é limitada, então $\overline{\left(T x_{n}\right)_{n \in \mathbb{N}}}$ é compacto, por definição. Logo, $\left(T x_{n}\right)_{n \in \mathbb{N}}$ possui subseqüência convergente.

Reciprocamente, se toda seqüência limitada $\left(x_{n}\right)_{n \in \mathbb{N}}$ possui subseqüência $\left(x_{n_{k}}\right)_{k \in \mathbb{N}}$ tal que $\left(T x_{n_{k}}\right)_{k \in \mathbb{N}}$ converge em $\mathcal{Y}$, então tomando $B \subset \mathcal{X}$ limitado e qualquer seqüência $\left(y_{n}\right)_{n \in \mathbb{N}}$ em $T(B)$, temos que para cada $n \in \mathbb{N}$ existe $x_{n} \in B$ tal que $y_{n}=T x_{n}$. Assim, por hipótese, $\left(T x_{n}\right)_{n \in \mathbb{N}}$ possui subseqüência convergente. Como $\left(y_{n}\right)_{n \in \mathbb{N}}=\left(T x_{n}\right)_{n \in \mathbb{N}}$ e $\left(y_{n}\right)_{n \in \mathbb{N}}$ foi tomada arbitrariamente, segue que $\overline{T(B)}$ é compacto.

6.3. Proposição. $\mathcal{K}$ é um ideal bilateral em $B(X)$.

Demonstração. $\mathcal{K}$ é um ideal à direita, pois se $K \in \mathcal{K}$ e $L \in B(X)$, é trivial que $K \circ L$ é compacto, já que se $A \subset X$ é limitado, então $L(A)$ é ainda limitado e, como $K$ é compacto, $\overline{K(L(A))}$ é compacto.

Por outro lado, se $\left(x_{n}\right)_{n \in \mathbb{N}}$ é um seqüência limitada em $X$, então, pelo lema acima, $\left(K x_{n}\right)_{n \in \mathbb{N}}$ possui uma subseqüência convergente $\left(K x_{n_{l}}\right)_{l \in \mathbb{N}}$. Mas $L$ é contínua e assim leva seqüência convergente em seqüência convergente; ou seja, $L\left(K x_{n_{l}}\right)$ é convergente. Novamente pela caracterização do lema acima, segue que $L \circ K$ também é compacto. Logo, segue tese. 
Finalmente, temos o seguinte resultado:

6.4. Teorema. Sejam $X$ e $Y$ espaços de Banach, então $\mathcal{K}$ é fechado em $B(X, Y)$.

Demonstração. Seja $K \in \overline{\mathcal{K}}$. Queremos ver que $K \in \mathcal{K}$. Como $B(X, Y)$ é métrico (pois é normado), existe uma seqüência $\left(K_{n}\right)_{n \in \mathbb{N}}$ em $\mathcal{K}$ tal que $\left\|K-K_{n}\right\| \longrightarrow 0$, quando $n \longrightarrow \infty$.

Seja $\left(x_{n}\right)_{n \in \mathbb{N}}$ uma seqüência limitada em $X$. Como $K_{1}$ é um operador compacto, existe $E_{1} \subset \mathbb{N}$ infinito tal que $\left(K_{1}\left(x_{n}\right)\right)_{n \in E_{1}}$ é convergente.

Como $\left(x_{n}\right)_{n \in \mathbb{N}}$ é limitada, então $\left(x_{n}\right)_{n \in E_{1}}$ também o é. Como $K_{2}$ é compacto, existe $E_{2} \subset E_{1}$ infinito tal que $\left(K_{2}\left(x_{n}\right)\right)_{n \in E_{2}}$ é convergente, etc. Desta forma, construímos uma seqüência encaixante de subconjuntos infinitos de $\mathbb{N}: E_{1} \supset E_{2} \supset \ldots \supset E_{k} \supset \ldots$ A partir dela definimos $z_{n}$ como sendo o $n$-ésimo elemento de $\left(x_{l}\right)_{l \in E_{n}}$. Segue então que $\left(z_{n}\right)_{n \in \mathbb{N}}$ está eventualmente em $E_{m}$, para todo $m \in \mathbb{N}$. Logo, para todo $m \in \mathbb{N},\left(K_{m}\left(z_{n}\right)\right)_{n \in \mathbb{N}}$ é um seqüência convergente e portanto de Cauchy.

Assim, tomando $\varepsilon>0$ arbitrário e $M:=\sup \left\{\left\|z_{n}\right\|: n \in \mathbb{N}\right\}<\infty$, existe $n_{0} \in \mathbb{N}$ tal que $\left\|K-K_{n_{0}}\right\|<\varepsilon /(3 M+1)$ e existe $m_{0} \in \mathbb{N}$ tal que $m, n>m_{0}$ implica $\left\|K_{n_{0}}\left(z_{m}\right)-K_{n_{0}}\left(z_{n}\right)\right\|<\varepsilon / 3$. Portanto, se $m, n>n_{0}$ :

$$
\begin{gathered}
\left\|K\left(z_{m}\right)-K\left(z_{n}\right)\right\| \leq\left\|K\left(z_{m}\right)-K_{n_{0}}\left(z_{m}\right)\right\|+\left\|K_{n_{0}}\left(z_{m}\right)-K_{n_{0}}\left(z_{n}\right)\right\|+ \\
+\left\|K_{n_{0}}\left(z_{n}\right)-K\left(z_{n}\right)\right\|<\left\|K-K_{n_{0}}\right\|\left\|\left|z_{m}\left\|+\frac{\varepsilon}{3}+\right\| K_{n_{0}}-K\|\|\right|\left(z_{n}\right)\right\| \\
<\frac{M}{3 M+1} \varepsilon+\frac{\varepsilon}{3}+\frac{M}{3 M+1} \varepsilon<\frac{\varepsilon}{3}+\frac{\varepsilon}{3}+\frac{\varepsilon}{3}=\varepsilon .
\end{gathered}
$$

Como $Y$ é completo, $\left(K z_{n}\right)_{n \in \mathbb{N}}$ é convergente e portanto $K \in \mathcal{K}$, como queríamos demonstrar.

Segue que $\mathcal{K}$ é um ideal bilateral fechado de $\mathcal{A}$ e portanto $\mathcal{A} / \mathcal{K}$ é também uma $C^{*}$-álgebra. Além disso, como $\mathcal{K}$ contém os comutadores de $\mathcal{A}$, segue que $\mathcal{A} / \mathcal{K}$ é uma $C^{*}$-álgebra comutativa, como queríamos demonstrar.

6.5. Observação. Nem todo elemento de $\mathcal{A}$ é compacto. Isto porque $I d=$ $M_{1} \in \mathcal{A}$, mas $I d$ não é um operador compacto, pois $\left(e_{n}\right)_{n \in \mathbb{N}}$ é uma seqüência limitada que não possui subseqüência convergente. Em particular, $\mathcal{A} / \mathcal{K}$ não é trivial. 
7. $M_{\mathcal{A} / \mathcal{K}}$ É Homeomorfo à Unĩ̃o Disjunta de Duas Cópias de $S^{1}$

Agora que já sabemos que $\mathcal{A} / \mathcal{K}$ é uma $C^{*}$-álgebra comutativa, vamos passar à tarefa de definir e descrever concretamente o seu espaço de Gelfand, $M_{\mathcal{A} / \mathcal{K}}$, mostrando que este é isomorfo a uma reunião disjunta de duas cópias de $S^{1}$.

7.1. Definição. Sejam $A$ e $B$ duas álgebras de Banach. Um homomorfismo $\phi: A \longrightarrow B$ é uma função linear que satisfaz:

$$
\forall a, b \in A, \phi(a b)=\phi(a) \phi(b)
$$

7.2. Notação. Seja $\mathcal{B}$ um álgebra de Banach. Denotaremos por $M_{\mathcal{B}}$ o conjunto dos homomorfismos não nulos de $\mathcal{B}$ em $\mathbb{C}$.

7.3. Notação. Denotaremos por $B^{*}$ o conjunto dos funcionais lineares contínuos de $B$ em $\mathbb{C}$, munido da topologia fraca-* (topologia da convergência pontual). E denotaremos por $B_{1}^{*}:=\left\{f \in B^{*}:\|f\| \leq 1\right\}$, o qual é compacto, pelo Teorema de Banach-Alaoglu. ${ }^{4}$

7.4. Proposição. Seja $\mathcal{B}$ uma álgebra de Banach com unidade. Então $M_{B}$ é um subespaço fechado de $B_{1}^{*}$, munido da topologia fraca-*.

Demonstração. Todo elemento de $M_{B}$ é um funcional linear. É necessário ver que os elementos de $M_{B}$ são limitados com norma menor ou igual a 1 , e que este subespaço é fechado.

De fato, sejam $w \in M_{B}$ e $x \in B$. Como, para todo $x \in B, w(x) \in \sigma(x)$ (o espectro de $x)$, pois $w(x-w(x))=0$ que não é inversível. Além disso, como ${ }^{5}$ $r(x) \leq\|x\|$, segue que $\|w(x)\| \leq\|x\|$, para todo $x \in B$. Logo, $\|w\| \leq 1$.

Além disso, $M_{B}$ é fechado em $B_{1}^{*}$, já que se $\left(w_{\alpha}\right)_{\alpha \in \Lambda}$ é uma rede ${ }^{6}$ em $M_{B}$ que converge para $w \in B_{1}^{*}$, então, usando que a multiplicação de números complexos é contínua e que o limite do produto é o produto dos limites,

\footnotetext{
${ }^{4}$ Para uma demosntração deste resultado veja [14]

${ }^{5}$ A demonstração deste resultado pode ser encontrada em [14], página 235. Sendo $r(x):=\sup \left\{|\lambda|:\left(\lambda 1_{\mathcal{B}}-x\right)\right.$ não é inversível $\}$ o raio espectral de $x$.

${ }^{6}$ Para uma rápida introdução ao uso de redes, veja o apêndice D.
} 
temos que:

$$
\begin{aligned}
w(x y) & =\lim w_{\alpha}(x y)=\lim \left(w_{\alpha}(x) w_{\alpha}(y)\right)=\lim \cdot\left(w_{\alpha}(x), w_{\alpha}(y)\right) \\
& =\cdot\left(\lim w_{\alpha}(x), \lim w_{\alpha}(y)\right)=\cdot(w(x), w(y)) \\
& =w(x) w(y) ;
\end{aligned}
$$

ou seja, $w \in M_{B}$. Segue que $M_{B}$ é fechado em $B_{1}^{*}$ e portanto compacto.

Em vista deste resultado, podemos formular a seguinte:

7.5. Definição. Dada uma álgebra de Banach com unidade $\mathcal{B}$, o espaço de Gelfand de $\mathcal{B}$ é o conjunto $M_{\mathcal{B}}$ munido da topologia fraca-*, induzida de $\mathcal{B}^{*}$.

7.6. Observação. A topologia fraca-* é a topologia da convergência pontual em $M_{\mathcal{B}}$ e as suas vizinhanças básicas são da forma:

$$
B_{\varepsilon}\left(f_{0}, x_{1}, \ldots, x_{n}\right):=\left\{g \in M_{\mathcal{B}}: \forall n \in\{1, \ldots, n\},\left|f_{0}\left(x_{i}\right)-g\left(x_{i}\right)\right|<\varepsilon\right\} .
$$

Assim, é fácil ver que a topologia fraca-* é Hausdorff.

Um resultado importante que vamos usar é o seguinte:

7.7. Teorema (Gelfand). Sejam $\mathcal{B}$ uma $C^{*}$-álgebra comutativa com unidade e $M_{\mathcal{B}}$ o seu espaço de Gelfand. Então:

$$
\begin{aligned}
\Lambda: \mathcal{B} & \longrightarrow C\left(M_{\mathcal{B}}\right) \\
x & \longmapsto \Lambda(x):=\operatorname{aval}_{x}: M_{\mathcal{B}} \longrightarrow \mathbb{C} \\
f & \longmapsto \operatorname{aval}_{x}(f):=f(x) .
\end{aligned}
$$

é um isomorfismo de $C^{*}$-álgebras.

Ou seja, dada uma $C^{*}$-álgebra $\mathcal{B}$ comutativa com unidade, temos que $\mathcal{B}$ é isomorfa a $C\left(M_{\mathcal{B}}\right)$. Além disso, temos o resultado 7.12, que é uma espécie de resultado dual do Teorema de Gelfand, mas antes de demonstrá-lo, vamos precisar dos seguintes lemas e da:

7.8. Definição. Seja $M$ um ideal de uma álgebra $A$. Dizemos que $M$ é um ideal maximal de $A$ se, e só se, $M$ é um ideal próprio e, para todo ideal $I$ :

$$
M \subset I \subset A \Rightarrow I=M \text { ou } I=A .
$$

7.9. Lema. Sejam $\Omega$ um espaço topológico compacto Hausdorff, $Y \subset \Omega e$ $\mathcal{I}(Y):=\{f \in C(\Omega): \forall y \in Y, f(y)=0\}$. Então os ideais maximais de $C(\Omega)$ são os conjuntos da forma $\mathcal{I}(\{w\})$, com $w \in \Omega$. 
Demonstração. Verificar que os conjuntos da forma $\mathcal{I}(Y)$ são ideais é trivial. Vejamos que os ideais maximais de $C(\Omega)$ são todos da forma $\mathcal{I}(\{w\})$.

Seja $I$ um ideal de $C(\Omega)$ e consideremos o conjunto $N(I):=\bigcap_{f \in I} Z(f)$, onde $Z(f):=\{x \in \Omega: f(x)=0\}$. Vejamos que: $N(I)=\emptyset \Rightarrow I=C(\Omega)$.

De fato, se $N(I)=\emptyset$, então, para cada $w \in \Omega$, existe $f_{w} \in I$ tal que $f_{w}(w) \neq 0$. Como cada $f_{w}$ é contínua, existe $V_{w}$ vizinhança de $w$ na qual elas não se anulam. Além disso, como $I$ é um ideal, $f_{w} \in I \Rightarrow \overline{f_{w}} f_{w}=\left|f_{w}\right|^{2} \in$ $I$, e assim podemos tomar as funções $f_{w}:=\left|f_{w}\right|^{2}$. Como $\Omega$ é compacto, existe uma sub-coleção finita $\left\{V_{w_{i}}\right\}_{i=1}^{n} \subset\left\{V_{w}\right\}_{w \in \Omega}$ tal que: $\bigcup_{i=1}^{n} V_{w_{i}}=\Omega$. Assim, temos que: $\sum_{i=1}^{n} f_{w_{i}}>0$ é um elemento inversível pertencente a $I$, e portanto: $I=C(\Omega)$, como queríamos.

Decorre disso que, para todo $x \in \Omega, \mathcal{I}(\{x\})$ é sempre um ideal maximal, pois se $\mathcal{I}(\{x\}) \subsetneq L$, então é claro que $N(L)=\emptyset$ (já que, pelo Lema de Urysohn, para todo $y \neq x$, existe $h \in \mathcal{I}(\{x\})$ tal que $h(y)=1)$. Isto dá conta de uma parte da caracterização.

Por outro lado, se $x, y \in N(I)$ e $x \neq y$, então $I \subsetneq \mathcal{I}(x) \subsetneq C(\Omega)$; ou seja, $I$ não é maximal. Logo, se $I$ é maximal, então $N(I)$ é unitário. Como $f \in I \Rightarrow f(x)=0$, então $I \subset \mathcal{I}(\{x\})$. Como $\mathcal{I}(\{x\})$ é próprio (pois é maximal), segue que: $I=\mathcal{I}(\{x\})$, como queríamos demonstrar.

7.10. Lema. Sejam $\mathcal{B}$ uma $C^{*}$-álgebra com unidade e $M_{\mathcal{B}}$ o seu espaço de Gelfand. $J$ é um ideal maximal de $\mathcal{B}$ se, e só se, existe $h \in M_{\mathcal{B}}$ tal que $J=\operatorname{ker}(h)$.

Demonstração. Seja $h \in M_{\mathcal{B}}$ e suponhamos que exista um ideal $L$ tal que: $\operatorname{ker}(h) \subsetneq L$. Mas o núcleo de um funcional não nulo tem sempre codimensão 1 e assim a classe de qualquer elemento $x_{\infty} \in L \backslash \operatorname{ker}(h)$ gera $\mathcal{B} / \operatorname{ker}(h)$. Disso decorre que se $\left\{x_{i}\right\}_{i \in I}$ é uma base de $\operatorname{ker}(h)$, então $\left\{x_{i}\right\}_{i \in I \cup\{\infty\}}$ é uma base de $\mathcal{B}$ que está contida em $L$. Logo, $\mathcal{B} / L=\{[0]\} ;$ ou seja, $\mathcal{B}=L$. Portanto, $\operatorname{ker}(h)$ é maximal.

Reciprocamente, se $M$ é um ideal maximal de $\mathcal{B}$, então $M$ é fechado ${ }^{7}$, e $\operatorname{assim} \mathcal{B} / M$ é uma álgebra de Banach. Seja $x \in \mathcal{B} \backslash M$ e consideremos o ideal:

$$
J:=\{a x+y: a \in \mathcal{B} \text { e } y \in M\} .
$$

\footnotetext{
${ }^{7}$ Já que $M \subset \bar{M} \subset \mathcal{B},\{$ inversíveis de $\mathcal{B}\}$ é aberto em $\mathcal{B}$ e $M \subset \mathcal{B} \backslash\{$ inversíveis de $\mathcal{B}$ \}.
} 
Temos que $M \subset J$, pois para todo $m \in M, m=0 x+m$; e $M \neq J$, pois $x=x 1+0 \in J$, o que quer dizer que $J=\mathcal{B}$ por causa da maximalidade de $M$. Assim, existem $a \in \mathcal{B}$ e $y \in M$ tais que: $a x+y=1$. Portanto, em $\mathcal{B} / M$

$$
[1]=[a x+y]=[a][x]+[y]=[a][x] .
$$

Logo, todo elemento não nulo em $\mathcal{B} / M$ é inversível e assim, pelo Teorema de Gelfand-Mazur ${ }^{8}, \mathcal{B} / M$ é isomorfa a $\mathbb{C}$.

Seja $j$ este isomorfismo. Então definindo $h:=j \circ[\cdot]$, obtemos um elemento de $M_{\mathcal{B}}$ cujo núcleo é $M$. Segue a tese.

7.11. Corolário. Existe uma bijeção entre os conjuntos: $\{\mathcal{I}(\{w\}): w \in \Omega\}$ $e\left\{\operatorname{ker}(h): h \in M_{C(\Omega)}\right\}$.

Demonstração. Pelos lemas anteriores, ambos têm uma bijeção com o conjunto: $\{I \subset C(\Omega): I$ é ideal maximal $\}$.

7.12. Proposição. Se $\Omega$ é um espaço compacto Hausdorff, então $\Omega$ é homeomorfo a $M_{C(\Omega)}$.

Demonstração. Considere a aplicação:

$$
\begin{aligned}
\text { aval }: \Omega & \longrightarrow M_{C(\Omega)} \\
w & \longmapsto \operatorname{aval}_{w}: C(\Omega) \\
& \longrightarrow \mathbb{C} \\
f & \longmapsto \operatorname{aval}_{x}(f):=f(x) .
\end{aligned}
$$

Precisamos mostrar que esta função é um homeomorfismo.

De fato, se $w_{\alpha} \longrightarrow w$ é uma rede convergente em $\Omega$, então, para todo $f \in C(\Omega)$ :

$$
\operatorname{aval}_{w_{\alpha}}(f)=f\left(w_{\alpha}\right) \longrightarrow f(w)=\operatorname{aval}_{w}(f) ;
$$

isto é, $\operatorname{aval}_{w_{\alpha}}$ converge ponto-a-ponto para $\operatorname{aval}_{w}$, que é a convergência da topologia fraca-* no espaço de Gelfand. Logo, aval é contínua.

Além disso, como $\Omega$ é um espaço compacto Hausdorff, $\Omega$ é normal, e, pelo Lema de Urysohn, dados $w, v \in \Omega$ com $w \neq v$, existe $f \in C(\Omega)$ tal que: $f(w)=1$ e $f(v)=0$. Logo, se $w \neq v$, aval $_{w} \neq \operatorname{aval}_{v}$; isto é, aval é injetora.

Só disto já decorre que aval é um homeomorfismo sobre a sua imagem, pois $\Omega$ é compacto e, pela observação $7.6, M_{C(\Omega)}$ é Hausdorff.

\footnotetext{
${ }^{8}$ Teorema 10.14 de [14] que afirma que: toda álgebra de Banach cujos elementos não nulos são inversíveis é isometricamante isomorfa a $\mathbb{C}$.
} 
Finalmente, decorre do corolário acima que para todo $w \in \Omega$ existe um, e apenas um, homomorfismo $h \in M_{C(\Omega)}$ tal que: $\operatorname{ker}(h)=\mathcal{I}(\{w\})$. Como $\operatorname{aval}_{w}$ faz este serviço, temos que $h=\operatorname{aval}_{w}$. Logo, aval é sobrejetora, e segue a tese.

Agora, lembrando que $\mathcal{M}$ e $\mathcal{D}$ estão definidas na página 14, e assumindo que $\Pi$ é a aplicação quociente módulo compactos, considere as seguintes funções:

$$
\begin{aligned}
i_{1}: C\left(S^{1}\right) & \longrightarrow \mathcal{A} / \mathcal{K} \\
a & \longmapsto i_{1}(a):=(\Pi \circ \mathcal{M})(a) \\
i_{2}: C(X) & \longrightarrow \mathcal{A} / \mathcal{K} \\
b & \longmapsto i_{2}(b):=(\Pi \circ \mathcal{D})(b) \\
\imath_{1}: M_{\mathcal{A} / \mathcal{K}} & \longrightarrow M_{C\left(S^{1}\right)} \\
w & \longmapsto \imath_{1}(w):=w \circ i_{1}=w \circ \Pi \circ \mathcal{M} \\
\imath_{2}: M_{\mathcal{A} / \mathcal{K}} & \longrightarrow M_{C(X)} \\
w & \longmapsto \imath_{2}(w):=w \circ i_{2}=w \circ \Pi \circ \mathcal{D} \\
\imath=\imath_{1} \times \imath_{2}: M_{\mathcal{A} / \mathcal{K}} & \longrightarrow M_{C\left(S^{1}\right)} \times M_{C(X)} \\
w & \longmapsto \imath_{(w)}=\left(\imath_{1}(w), \imath_{1}(w)\right)=\left(w \circ i_{1}, w \circ i_{2}\right) .
\end{aligned}
$$

Como $M_{C\left(S^{1}\right)} \cong S^{1}$ e $M_{C(X)} \cong X$, para cada $w \in M_{\mathcal{A} / \mathcal{K}}$ existem $z \in S^{1}$ e $j \in X$ tais que $\left(w \circ i_{1}, w \circ i_{2}\right)=\left(\operatorname{aval}_{z}, \operatorname{aval}_{j}\right)$. Agora, mostrar que:

7.13. Proposição. $\imath$ é um homeomorfismo sobre a sua imagem. ${ }^{9}$

Demonstração. Como $M_{\mathcal{A} / \mathcal{K}}$ é compacto e $M_{C\left(S^{1}\right)} \times M_{C(X)}$ é Hausdorff, basta ver que $\imath$ é contínua e injetora, pois daí decorrerá que $\imath$ é fechada e bijetora sobre a sua imagem. Vejamos:

Seja $w_{\alpha} \longrightarrow w$ uma rede convergente em $M_{\mathcal{A} / \mathcal{K}}$; isto é, pela definição da topologia fraca-*, para todo $x \in \mathcal{A} / \mathcal{K}, w_{\alpha}(x) \longrightarrow w(x)$ em $\mathbb{C}$. Em particular, como para todo $a \in C\left(S^{1}\right), i_{1}(a) \in M_{\mathcal{A} / \mathcal{K}}$, temos que $w_{\alpha}\left(i_{1}(a)\right) \longrightarrow$

\footnotetext{
${ }^{9} \mathrm{~A}$ idéia desta demonstração é a mesma daquela do Lema de Herman, do qual este resultado é um caso particular. Para a demosntração do Lema de Herman, veja [3], página 137 .
} 
$w\left(i_{1}(a)\right)$, e analogamente $w_{\alpha}\left(i_{2}(a)\right) \longrightarrow w\left(i_{2}(a)\right)$. Isto é, $\imath\left(w_{\alpha}\right) \longrightarrow \imath(w)$, pois uma rede num produto cartesiano é convergente se, e só se, as suas coordenadas o são. Como uma função é continua se, e só se, leva rede convergente em rede convergente, segue que $\imath$ é contínua.

Além disso, se $\imath(w)=\imath(v)$, então $w \circ i_{1}=w \circ i_{1}$ e $w \circ i_{2}=w \circ i_{2}$. Logo, para todo $a \in C\left(S^{1}\right)$ e para todo $b \in C(X)$, temos que $w\left(i_{1}(a)\right)=w\left(i_{1}(a)\right)$ e $w\left(i_{2}(b)\right)=w\left(i_{2}(b)\right)$. Portanto, $w$ e $v$ coincidem na álgebra gerada pelas imagens de $i_{1}$ e $i_{2}$, que é densa em $\mathcal{A} / \mathcal{K}$. Como $w$ e $v$ são funções contínuas, segue que $w=v$.

Logo, $\imath$ é um homeomorfismo sobre a sua imagem, como queríamos demonstrar.

7.14. Definição. Vamos agora definir a seguinte aplicação:

$$
\begin{aligned}
L: C(\operatorname{Im}(\imath)) & \longrightarrow C\left(M_{\mathcal{A} / \mathcal{K}}\right) \\
f & \longmapsto L(f):=f \circ \imath
\end{aligned}
$$

7.15. Observação. Como $\imath$ é um homeomorfismo sobre a sua imagem, fica claro que $L$ é um $*$-isomorfismo cuja inversa é

$$
\begin{aligned}
L^{-1}: C\left(M_{\mathcal{A} / \mathcal{K}}\right) & \longrightarrow C(\operatorname{Im}(\imath)) \\
g & \longmapsto L^{-1}(g):=g \circ \imath^{-1} .
\end{aligned}
$$

7.16. Definição. A seguinte composição de funções $\sigma:=L^{-1} \circ \Lambda \circ \Pi$ ilustrada pelo diagrama abaixo

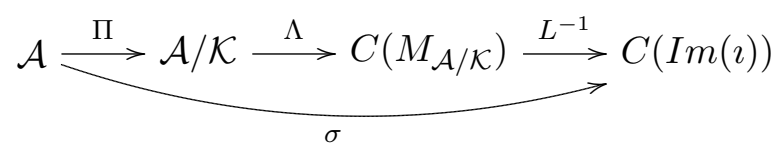

será chamada função símbolo.

Como $L^{-1}, \Lambda$ e $\Pi$ são $*$-homomorfismos, $\sigma$ também o é. Além disso, com $L^{-1}, \Lambda$ são isomorfismos, $\sigma(A)=0$ se, e só se, $\Pi(A)=0$, ou seja, se, e só se, $A$ é compacto. Isto é, $\operatorname{Ker}(\sigma)=\mathcal{K}$.

7.17. Lema. Sejam $a \in C\left(S^{1}\right), b \in C(X), z \in S^{1}$ e $j \in X$. Então

$$
\begin{aligned}
& \sigma\left(M_{a}\right)\left(\text { aval }_{z}, \text { aval }_{j}\right)=a(z) ; e \\
& \sigma\left(D_{b}\right)\left(\text { aval }_{z}, \text { aval }_{j}\right)=b(j) .
\end{aligned}
$$


Demonstração. Pela definição do símbolo, temos que $\sigma\left(M_{a}\right)=\left(L^{-1} \circ \Lambda \circ\right.$ $\Pi)\left(M_{a}\right)$, para todo $a \in C\left(S^{1}\right)$. Então, $(L \circ \sigma)\left(M_{a}\right)=(\Lambda \circ \Pi)\left(M_{a}\right)$. Assim, se $w \in M_{\mathcal{A} / \mathcal{K}}$ é tal que $\imath(w)=\left(\operatorname{aval}_{z}, \operatorname{aval}_{j}\right)$, por um lado,

$$
\begin{aligned}
\left((L \circ \sigma)\left(M_{a}\right)\right)(w) & =\left(\sigma\left(M_{a}\right) \circ \imath\right)(w) \\
& =\sigma\left(M_{a}\right)(\imath(w)) \\
& =\sigma\left(M_{a}\right)\left(\operatorname{aval}_{z}, \operatorname{aval}_{j}\right) ;
\end{aligned}
$$

por outro lado,

$$
\begin{aligned}
\left((\Lambda \circ \Pi)\left(M_{a}\right)\right)(w) & =\left(\left(\Lambda\left(\left[M_{a}\right]\right)\right)(w)=\operatorname{aval}_{\left[M_{a}\right]}(w)=w\left(\left[M_{a}\right]\right)\right. \\
& =(w \circ \Pi \circ \mathcal{M})(a)=w\left(i_{1}(a)\right) \\
& =\imath_{1}(w)(a)=\operatorname{aval}_{z}(a) \\
& =a(z) .
\end{aligned}
$$

Logo, segue que $\sigma\left(M_{a}\right)\left(\operatorname{aval}_{z}, \operatorname{aval}_{j}\right)=a(z)$, como queríamos demonstrar. A outra igualdade é completamente análoga.

O Espaço de Gelfand $M_{\mathcal{A} / \mathcal{K}}$. Daqui para frente, passaremos a caracterizar a imagem de $\imath$. Levando-se em consideração o homeomorfismo $S^{1} \times X \cong M_{C\left(S^{1}\right)} \times M_{C(X)}$, que a cada par $(z, j)$ associa $\left(\operatorname{aval}_{z}\right.$, aval $\left._{j}\right)$, vamos mostrar que $\operatorname{Im}(\imath) \cong S^{1} \times\{-\infty,+\infty\}$. Para este fim, vamos precisar de três lemas.

7.18. Lema. $\operatorname{Im}(\imath) \subset\left\{\operatorname{aval}_{z}: z \in S^{1}\right\} \times\left\{\operatorname{aval}_{-\infty}, \operatorname{aval}_{+\infty}\right\} \cong S^{1} \times\{-\infty,+\infty\}$. Demonstração. Suponhamos que não. Então existiriam $j \in \mathbb{Z}, z \in S^{1}$ e $w \in$ $M_{\mathcal{A} / \mathcal{K}}$ tais que $\imath(w)=\left(\operatorname{aval}_{z}, \operatorname{aval}_{j}\right)$. Mas então consideremos $b:=\left(\delta_{i j}\right)_{i \in \mathbb{Z}} \in$ $C(X)$. É claro que $D_{b}$ é um operador compacto, portanto $\sigma\left(D_{b}\right)$ é a função nula. Por outro lado, vimos, pelo lema, que $\sigma\left(D_{b}\right)\left(\operatorname{aval}_{z}, \operatorname{aval}_{j}\right)=b(j)=1$, o que é uma contradição. Logo, segue a tese.

7.19. Lema. Sejam $v \in S^{1} e\left(\operatorname{aval}_{z}, \operatorname{aval}_{j}\right) \in \operatorname{Im}(\imath)$, então $\left(\operatorname{aval}_{v z}, \operatorname{aval}_{j}\right) \in$ $\operatorname{Im}(\imath)$.

Demonstração. Primeiro definimos a função:

$$
\begin{aligned}
T_{v}: L^{2}\left(S^{1}\right) & \longrightarrow L^{2}\left(S^{1}\right) \\
f & \longmapsto T_{v}(f): S^{1} \longrightarrow \mathbb{C} \\
z & \longmapsto\left(T_{v}(f)\right)(z):=f(v z) .
\end{aligned}
$$


Agora, dado $w \in M_{\mathcal{A} / \mathcal{K}}$ tal que $\imath(w)=\left(\operatorname{aval}_{z}, \operatorname{aval}_{j}\right) \in \operatorname{Im}(\imath)$, vamos definir $\widetilde{w} \in M_{\mathcal{A} / \mathcal{K}}$ de forma que se $[A] \in \mathcal{A} / \mathcal{K}$, então $\widetilde{w}([A]):=w\left(\left[T_{v^{-1}} A T_{v}\right]\right)$.

Vamos verificar que $\widetilde{w}$ está bem definido, ou seja, que para cada $v \in S^{1}$ $T_{v^{-1}} A T_{v} \in \mathcal{A}$. Primeiramente verificaremos que:

$$
\begin{gathered}
T_{v^{-1}} M_{a} T_{v}=M_{a_{v^{-1}}} \\
T_{v^{-1}} D_{b} T_{v}=D_{b},
\end{gathered}
$$

onde $a_{v^{-1}}(z):=a\left(v^{-1} z\right)$, para todo $z \in S^{1}$. (É claro que $a_{v^{-1}}$ é contínua.)

Para mostrar estas igualdades, primeiro observamos que $T_{v}^{-1}=T_{v^{-1}}$.

Agora, sejam $f \in L^{2}\left(S^{1}\right)$ e $z \in S^{1}$. Então, a primeira igualdade segue de:

$$
M_{a} T_{v}(f)(z)=a(z) f(v z)=a\left(v v^{-1} z\right) f(v z)=T_{v} M_{a_{v^{-1}}}(f)(z) .
$$

Para a segunda igualdade, tomando $v^{\mathrm{J}}:=\left(v^{j}\right)_{j \in \mathbb{Z}} \in l^{\infty}(\mathbb{Z})$, notemos que $F T_{v}=M_{v^{\mathrm{s}}} F$. De fato, se escrevermos $v=e^{i y}$ e fizermos uma mudança de variável, $\operatorname{com} x+y=: w$, então:

$$
\begin{aligned}
F T_{v}(f)(z) & =\left(\int_{-\pi}^{\pi} e^{-i j x} \tilde{f}(x+y) d x\right)_{j \in \mathbb{Z}} \\
& =\left(\int_{-\pi}^{\pi} e^{-i j(w-y)} \tilde{f}(w) d w\right)_{j \in \mathbb{Z}} \\
& \left.=\left(\int_{-\pi}^{\pi} e^{i j y} e^{-i j w} \tilde{f}(w)\right) d w\right)_{j \in \mathbb{Z}} \\
& \left.=\left(v^{j} \int_{-\pi}^{\pi} e^{-i j w} \tilde{f}(w)\right) d w\right)_{j \in \mathbb{Z}} \\
& =M_{v^{\mathrm{j}}} F(f)(z) .
\end{aligned}
$$

Então, como $F T_{v}=M_{v^{j}} F$, temos que:

$$
\begin{aligned}
T_{-v} D_{b} T_{v} & =T_{-v} F^{-1} M_{b} F T_{v}=\left(F T_{v}\right)^{-1} M_{b}\left(F T_{v}\right) \\
& =\left(M_{v^{\mathrm{J}}} F\right)^{-1} M_{b} M_{v^{\mathrm{J}}} F=F^{-1} M_{v^{-\mathrm{J}}} M_{b} M_{v^{\mathrm{J}}} F \\
& =F^{-1} M_{v^{-\mathrm{J}} b v^{\mathrm{J}}} F=F^{-1} M_{b} F \\
& =D_{b}
\end{aligned}
$$

Agora, seja $L \in \mathcal{A}$. Então existe uma seqüência $\left(L_{n}\right)_{n \in \mathbb{N}}$ de elementos da álgebra finitamente gerada $\mathcal{A}_{f}$ que converge para $L$ na norma de $\mathcal{A}$. Como 
a operação que a cada $S \in \mathcal{A}$ associa $T_{-v} S T_{v}$ é uma isometria (pois $T_{v}$ é unitário) temos que:

$$
\lim _{n \rightarrow \infty} T_{-v} L_{n} T_{v}=T_{-v}\left(\lim _{n \rightarrow \infty} L_{n}\right) T_{v}=T_{-v} L T_{v} \in \mathcal{A} .
$$

Ou seja, vimos que $\widetilde{w}$ está bem definido. Além disso, é claro que $\tilde{w}$ é um homomorfismo, logo $\widetilde{w} \in M_{\mathcal{A} / \mathcal{K}}$. Resta ver que $\imath(\tilde{w})=\left(\operatorname{aval}_{v^{-1} z}, \operatorname{aval}_{j}\right)$.

De fato, $\imath(\tilde{w})=\left(\tilde{w} \circ i_{1}, \tilde{w} \circ i_{2}\right)$, e como $\operatorname{aval}_{z}=w \circ \Pi \circ \mathcal{M}$, segue que, para todo $a \in C\left(S^{1}\right)$ :

$$
\begin{aligned}
\tilde{w} \circ i_{1}(a) & =\tilde{w} \circ \Pi \circ \mathcal{M}(a)=\tilde{w}\left(\left[M_{a}\right]\right) \\
& =w\left(\left[T_{v^{-1}} M_{a} T_{v}\right]\right)=w\left(\left[M_{a_{v^{-1}}}\right]\right) \\
& =w \circ \Pi \circ \mathcal{M}\left(a_{v^{-1}}\right)=\operatorname{aval}_{z}\left(a_{v^{-1}}\right) \\
& =a_{v^{-1}}(z)=a\left(v^{-1} z\right) \\
& =\operatorname{aval}_{v^{-1} z}(a) .
\end{aligned}
$$

Por outro lado, como $\operatorname{aval}_{j}=w \circ \Pi \circ \mathcal{D}$, segue que, para todo $b \in C(X)$ :

$$
\begin{aligned}
\tilde{w} \circ i_{2}(b) & =\tilde{w} \circ \Pi \circ \mathcal{D}(b)=\tilde{w}\left(\left[D_{b}\right]\right) \\
& =w\left(\left[T_{v^{-1}} D_{b} T_{v}\right]\right)=w\left(\left[D_{b}\right]\right) \\
& =w \circ \Pi \circ \mathcal{D}(b)=\operatorname{aval}_{j}(b) \\
& =b(j) \\
& =\operatorname{aval}_{j}(b) .
\end{aligned}
$$

Tomando $v^{-1}$ no lugar de $v$ na construção de $\tilde{w}$, temos que: $\imath(\tilde{w})=$ $\left(\operatorname{aval}_{v z}, \operatorname{aval}_{j}\right)$; ou seja, $\left(\operatorname{aval}_{v z}, \operatorname{aval}_{j}\right) \in \operatorname{Im}(\imath)$, como queríamos demonstrar.

7.20. Lema. Se $z \in S^{1}$ e $j \in X$ são tais que $\left(\operatorname{aval}_{z}, \operatorname{aval}_{j}\right) \in \operatorname{Im}(\imath)$, então $\left(\operatorname{aval}_{\bar{z}}, \operatorname{aval}_{-j}\right) \in \operatorname{Im}(\imath)$.

Demonstração. Consideremos a seguinte função:

$$
\begin{aligned}
B: l^{2}(\mathbb{Z}) & \longrightarrow l^{2}(\mathbb{Z}) \\
\left(c_{n}\right)_{n \in \mathbb{Z}} & \longmapsto B\left(\left(c_{n}\right)_{n \in \mathbb{Z}}\right):=\left(c_{-n}\right)_{n \in \mathbb{Z}}
\end{aligned}
$$

É claro que $B$ é um $*$-isomorfismo isométrico, com $B^{-1}=B$. Portanto, $G:=F^{-1} B F$ também o é. 
Vejamos que se $T \in \mathcal{A}$, então $G T G \in \mathcal{A}$ e que, se $\left(\operatorname{aval}_{z}, \operatorname{aval}_{j}\right) \in \operatorname{Im}(\imath)$, com $w \in M_{\mathcal{A} / \mathcal{K}}$, é tal que $\imath(w)=\left(\operatorname{aval}_{z}, \operatorname{aval}_{j}\right)$, então $\tilde{w}$, que a cada $T \in \mathcal{A}$ associa $\tilde{w}([A]):=w([G A G])$, pertence a $M_{\mathcal{A} / \mathcal{K}}$ e $\imath(\tilde{w})=\left(\right.$ aval $_{\bar{z}}$, aval $\left._{-j}\right)$.

Inicialmente, observamos que:

$$
\begin{aligned}
B F(f) & =B\left(\int_{-\pi}^{\pi} e^{-i j x} f\left(e^{i x}\right) d x\right)_{j \in \mathbb{Z}} \\
& =\left(\int_{-\pi}^{\pi} e^{i j x} f\left(e^{i x}\right) d x\right)_{j \in \mathbb{Z}} \\
& =\left(\int_{\pi}^{-\pi} e^{-i j w} f\left(e^{-i w}\right)(-1) d w\right)_{j \in \mathbb{Z}} \\
& =\left(\int_{-\pi}^{\pi} e^{-i j w}(f \circ c)\left(e^{i w}\right) d w\right)_{j \in \mathbb{Z}} \\
& =F C(f),
\end{aligned}
$$

onde $c: \mathbb{C} \longrightarrow \mathbb{C}$ é a função conjugado que a cada $z \in \mathbb{C}$ associa $c(z):=\bar{z}$, e $C: L^{2}\left(S^{1}\right) \longrightarrow L^{2}\left(S^{1}\right)$ é a função que a cada $f \in L^{2}\left(S^{1}\right)$ associa $C(f):=$ $f \circ c$. Disso segue que:

$$
\begin{aligned}
G M_{a} G(f) & =G M_{a} F^{-1} B F(f) \\
& =G M_{a} F^{-1} F C(f)=F^{-1} B F M_{a} C(f) \\
& =F^{-1} F C M_{a} C(f)=C M_{a} C(f) \\
& =C M_{a}(f \circ c)=C(a \cdot(f \circ c)) \\
& =(a \circ c) \cdot(f \circ c \circ c)=(a \circ c) \cdot f \\
& =M_{a \circ c}(f) ;
\end{aligned}
$$

ou seja, $G M_{a} G=M_{a \circ c} \in \mathcal{A}$.

Por outro lado, se $d:=\left(b_{-j}\right)_{j \in \mathbb{Z}}$, então:

$$
\begin{aligned}
G D_{b} G(f) & =F^{-1} B F F^{-1} M_{b} F F^{-1} B F(f)=F^{-1} B M_{b} B F(f) \\
& =F^{-1} B M_{b}\left(\left(F(f)_{-j}\right)_{j \in \mathbb{Z}}\right)=F^{-1} B\left(\left(b_{j} F(f)_{-j}\right)_{j \in \mathbb{Z}}\right) \\
& =F^{-1}\left(\left(b_{-j} F(f)_{j}\right)_{j \in \mathbb{Z}}\right)=F^{-1} M_{d} F(f) \\
& =D_{d}(f) .
\end{aligned}
$$

Logo, $G D_{b} G=D_{d} \in \mathcal{A}$.

Como $\|G S G\|=\|S\|$, temos que a transformação que a cada $S \in \mathcal{A}$ associa $G S G$ é contínua e portanto, se $\left(T_{n}\right)_{n \in \mathbb{N}}$ é uma seqüência em $\mathcal{A}_{f}$ que 
converge para $T \in \mathcal{A}$, então:

$$
\lim _{n \rightarrow \infty} G T_{n} G=G\left(\lim _{n \rightarrow \infty} T_{n}\right) G=G T G \in \mathcal{A},
$$

o que mostra que $\tilde{w}$ está bem definido em $\mathcal{A}$.

Vamos agora avaliar $\imath(\tilde{w})$. Seja $a \in C\left(S^{1}\right)$. Como $\imath(\tilde{w})=\left(\tilde{w} \circ i_{1}, \tilde{w} \circ i_{2}\right)$, temos que:

$$
\begin{aligned}
\tilde{w} \circ i_{1}(a) & =\tilde{w} \circ \Pi \circ \mathcal{M}(a)=\tilde{w}\left(\left[M_{a}\right]\right) \\
& =w\left(\left[G M_{a} G\right]\right)=w\left(\left[M_{a \circ c}\right]\right) \\
& =w \circ \Pi \circ \mathcal{M}(a \circ c)=\operatorname{aval}_{z}(a \circ c) \\
& =a \circ c(z)=a(\bar{z}) \\
& =\operatorname{aval}_{\bar{z}}(a)
\end{aligned}
$$

Por outro lado, se $b \in C(X)$, então:

$$
\begin{aligned}
\tilde{w} \circ i_{2}(b) & =\tilde{w} \circ \Pi \circ \mathcal{D}(b)=\tilde{w}\left(\left[D_{b}\right]\right) \\
& =w\left(\left[G D_{b} G\right]\right)=w\left(\left[D_{d}\right]\right) \\
& =w \circ \Pi \circ \mathcal{D}(d)=\operatorname{aval}_{j}(d) \\
& =d(j)=b(-j) \\
& =\operatorname{aval}_{-j}(b) .
\end{aligned}
$$

Ou seja, $\imath(\tilde{w})=\left(\operatorname{aval}_{\bar{z}}\right.$, aval $\left._{-j}\right)$, como queríamos demonstrar.

Agora usaremos os três lemas anteriores para provar o resultado desta seção:

7.21. Teorema. $M_{\mathcal{A} / \mathcal{K}}$ é homeomorfo a $S^{1} \times\{-\infty,+\infty\}$.

Demonstração. $\operatorname{Dom}(\imath) \neq \emptyset$, então $\operatorname{Im}(\imath) \neq \emptyset$. Pelo lema 7.18 sabemos que, para algum $z \in S^{1},\left(\operatorname{aval}_{z}, \operatorname{aval}_{-\infty}\right)$ ou $\left(\operatorname{aval}_{z}, \operatorname{aval}_{\infty}\right)$ pertence a $\operatorname{Im}(\imath)$. Podemos então supor sem perda de generalidade que $\left(\operatorname{aval}_{z}, \operatorname{aval}_{\infty}\right) \in \operatorname{Im}(\imath)$.

Pelo lema 7.19, temos que $\left(\operatorname{aval}_{v z}, \operatorname{aval}_{\infty}\right) \in \operatorname{Im}(\imath)$, para todo $v \in S^{1}$. Logo, $\left\{\operatorname{aval}_{z}: z \in S^{1}\right\} \times\left\{\operatorname{aval}_{\infty}\right\} \subset \operatorname{Im}(\imath)$.

Mas, pelo lema 7.20, temos que $\left(\operatorname{aval}_{\bar{z}}\right.$, aval $\left._{-\infty}\right) \in \operatorname{Im}(\imath)$, para todo $z \in S^{1}$. Logo, pelo mesmo argumento, $\left\{\operatorname{aval}_{z}: z \in S^{1}\right\} \times\left\{\operatorname{aval}_{-\infty}\right\} \subset \operatorname{Im}(\imath)$.

Como já havíamos estabelecido a inclusão oposta em 7.18, segue que:

$$
\operatorname{Im}(\imath)=\left\{\operatorname{aval}_{z}: z \in S^{1}\right\} \times\left\{\text { aval }_{-\infty}, \operatorname{aval}_{\infty}\right\} \cong S^{1} \times\{-\infty,+\infty\},
$$

onde o homeomorfismo acima é aquele da proposição 7.12. 


\section{O Índice de Fredholm de $T \in \mathcal{A}$ a partir do seu Símbolo}

Já sabemos que o espaço de Gelfand de $\mathcal{A} / \mathcal{K}, M_{\mathcal{A} / \mathcal{K}}$, é isomorfo a uma reunião disjunta de duas cópias de $S^{1}$.

Nesta seção, vamos definir os operadores de Fredholm e o índice de tais operadores. O objetivo é mostrar que um operador $T \in \mathcal{A}$ é de Fredholm se, e só se, o símbolo de $\mathrm{T}$ possui inverso multiplicativo. Além disso, daremos uma fórmula para calcular o índice de um operador conhecendo-se o número de rotação de cada uma das restrições de $\sigma$ a $S^{1} \times\{-\infty\}$ e a $S^{1} \times\{+\infty\}$, denotadas por $\sigma^{-}(T)$ e $\sigma^{+}(T)$, respectivamente. Nosso objetivo é provar a seguinte fórmula:

$$
\operatorname{ind}(T)=\operatorname{wind} \#\left(\sigma^{+}(T)\right)-\operatorname{wind} \#\left(\sigma^{-}(T)\right),
$$

onde wind\# denota o número de rotação em torno da origem de uma curva contínua de $S^{1}$ em $\mathbb{C} \backslash\{0\}$.

8.1. Definição. Sejam $X$ e $Y$ espaços vetoriais. Uma transformação linear $T: X \longrightarrow Y$ é chamada de Fredholm se, e só se, $\operatorname{dim}(\operatorname{ker}(T))<\infty$ e $\operatorname{dim}\left(\frac{Y}{\operatorname{Im}(T)}\right)<\infty$. Denotaremos o conjunto dos operadores de Fredholm por $\mathcal{F}(X, Y)$.

8.2. Definição. Definimos o índice de um operador de Fredholm $T$ como sendo a diferença: $\operatorname{dim}(\operatorname{ker}(T))-\operatorname{dim}\left(\frac{Y}{\operatorname{Im}(T)}\right)=: \operatorname{ind}(T) \in \mathbb{Z}$.

8.3. Definição. Seja $T \in \mathcal{A}$. Definimos $\sigma^{-}(T)$ e $\sigma^{+}(T)$ como sendo as retrições de $\sigma(T)$ a $S^{1} \times\{-\infty\}$ e a $S^{1} \times\{+\infty\}$, respectivamente.

8.4. Teorema. $T \in \mathcal{A}$ é de Fredholm se, e só se, $\sigma(T)$ nunca se anula.

Demonstração. Como vimos que $\sigma(T)$ é contínua, segue que $\sigma^{-}(T)$ e $\sigma^{+}(T)$ também o são.

Pelo Teorema de Atkinson ${ }^{10}, T \in \mathcal{B}(\mathcal{H})$ é de Fredholm se, e só se, $[T]$ é inversível em $\mathcal{B}(\mathcal{H}) / \mathcal{K}$. Além disso, se $T \in \mathcal{A}$, $[T]$ é inversível em $\mathcal{A} / \mathcal{K}$ se ${ }^{11}$, e só se, $[T]$ é inversível em $\mathcal{B}(\mathcal{H}) / \mathcal{K}$, pois $\mathcal{A} / \mathcal{K}$ é uma $C^{*}$-subálgebra de $\mathcal{B}(\mathcal{H}) / \mathcal{K}$. Logo, $T \in \mathcal{A}$ é de Fredholm se, e só se, $[T] \in \mathcal{A} / \mathcal{K}$ é inversível.

\footnotetext{
${ }^{10}$ Ver [5], página 114 .

${ }^{11}$ Se $\mathcal{B}$ uma $C^{*}$-álgebra e $\mathcal{A}$ é uma $C^{*}$-subálgebra de $\mathcal{B}$, então: $a \in \mathcal{A}$ e $a^{-1} \in \mathcal{B} \Rightarrow$ $a^{-1} \in \mathcal{A}$. Para uma demosntração disto, veja [13], página 41.
} 
Por outro lado, os elementos inversíveis de $C(\operatorname{Im}(\imath))$ são as funções que nunca se anulam.

Como $L^{-1} \circ \Lambda$ é uma bijeção entre os inversíveis, já que é um isomorfismo, segue o resultado.

Assim, faz sentido considerar o número de rotação destas duas funções. O nosso objetivo agora é mostrar que:

$$
\operatorname{ind}(T)=\operatorname{wind} \#\left(\sigma^{+}(T)\right)-\operatorname{wind} \#\left(\sigma^{-}(T)\right) .
$$

Para isto, vamos precisar do seguinte lema:

8.5. Lema (Levantamento). Sejam A uma $C^{*}$-álgebra, $J$ um ideal bilateral fechado de $A$ e $\Pi: A \longrightarrow A / J$ a aplicação quociente. Sejam $x, y \in A e$ $\alpha:[0,1] \longrightarrow A / J$ um caminho contínuo ligando $[x]$ a $[y]$. Então existe um caminho contínuo $\beta:[0,1] \longrightarrow$ A tal que $\beta(0)=x, \beta(1)=y$ e $\Pi \circ \beta=\alpha$.

Demonstração. Ver o apêndice B.

O lema acima nos diz que se o ideal é fechado, então uma curva contínua no quociente pode ser levantada para uma curva contínua na álgebra. Vamos usar este fato para provar a seguinte proposição.

8.6. Proposição. Se $\sigma(T)$ é homotópica a $\sigma(S)$ nos inversiveis de $C\left(S^{1} \times\right.$ $\{-\infty,+\infty\})$, então ind $(T)=\operatorname{ind}(S)$.

Demonstração. Seja $\gamma$ uma homotopia ligando $\sigma(T)$ a $\sigma(S)$. Então, como $\Lambda^{-1} \circ L$ é um $*$-isomorfismo, $\Lambda^{-1} \circ L \circ \gamma$ é uma função contínua. Ou seja, uma homotopia entre $\sigma(T)$ e $\sigma(S)$ induz um caminho contínuo nos inversíveis de $\mathcal{A} / \mathcal{K}$ ligando $[T]$ a $[S]$.

Então, tomando $\alpha:=\Lambda^{-1} \circ L \circ \gamma$ e notando que $\mathcal{K}$ é um ideal fechado de $\mathcal{A}$, pelo lema 8.5 acima existe $\beta$ caminho contínuo de $[0,1]$ nos inversíveis de $\mathcal{A}$ tal que $\beta(0)=T$ e $\beta(1)=S$. Logo, $T$ e $S$ são operadores de Fredholm unidos por uma curva contínua de operadores de Fredholm. Como o índice é localmente constante, ${ }^{12}$ segue que: $\operatorname{ind}(T)=\operatorname{ind}(S)$.

8.7. Proposição. Para cada par $(l, k) \in \mathbb{Z}^{2}$, existe $T_{k, l} \in \mathcal{A}$ tal que:

$$
\text { wind } \#\left(\sigma^{+}(T)\right)=k \text { e wind\# }\left(\sigma^{-}(T)\right)=l .
$$

\footnotetext{
${ }^{12}$ Ver [3], página 266.
} 
Demonstração. Seja $\chi^{k} \in C\left(S^{1}\right)$ a função que a cada $z \in S^{1}$ associa $\chi^{k}(z):=$ $z^{k} \in \mathbb{C}$. Tomando $M_{\chi^{k}}$, temos que, para todo $(z, j) \in S^{1} \times\{-\infty,+\infty\}$, $\sigma\left(M_{\chi^{k}}\right)(z, j)=z^{k}$.

Por outro lado, se considerarmos $b \in C(X)$ dada por

$$
b(j):= \begin{cases}0, & \text { se } n \leq 0 \\ 1, & \text { se } n>0\end{cases}
$$

então temos que, para todo $(z, j) \in S^{1} \times\{-\infty,+\infty\}, \sigma\left(D_{b}\right)(z, j)=b(j)$. Logo, tomando $T_{k, l}=M_{\chi^{k}} D_{b}+M_{\chi^{l}}\left(I d-D_{b}\right)$, temos que, para todo $(z, j) \in$ $S^{1} \times\{-\infty,+\infty\}, \sigma\left(T_{k, l}\right)(z, j)=z^{k} b(j)+z^{l}(1-b(j))$. Assim,

$$
\begin{aligned}
& \sigma^{+}\left(T_{k, l}\right)(z)=z^{k} b(+\infty)+z^{l}(1-b(+\infty))=z^{k} 1+z^{l} 0=z^{k} \\
& \sigma^{-}\left(T_{k, l}\right)(z)=z^{k} b(-\infty)+z^{l}(1-b(-\infty))=z^{k} 0+z^{l} 1=z^{l}
\end{aligned}
$$

Portanto,

$$
\begin{aligned}
& \operatorname{wind} \#\left(\sigma^{+}\left(T_{k, l}\right)\right)=k \\
& \operatorname{wind} \#\left(\sigma^{-}\left(T_{k, l}\right)\right)=l
\end{aligned}
$$

como queríamos demonstrar.

8.8. Proposição. Seja $T_{k, l}=M_{\chi^{k}} D_{b}+M_{\chi^{l}}\left(I d-D_{b}\right)$. Então, $\operatorname{Ind}\left(T_{k, l}\right)=$ $k-l$.

Demonstração. Inicialmente observemos que, como $\operatorname{Ind}(A B)=\operatorname{Ind}(A)+$ $\operatorname{Ind}(B)$ (ver [3], página 262), $\operatorname{Ind}\left(T_{k, l}\right)=\operatorname{Ind}\left(T_{k+m, l+m}\right)$, pois $T_{k+m, l+m}=$ $M_{\chi^{m}} T_{k, l}$ e $\operatorname{Ind}\left(M_{\chi^{m}}\right)=0$, já que $M_{\chi^{m}}$ é um isomorfismo. $\operatorname{Logo}, \operatorname{Ind}\left(T_{k, l}\right)=$ $\operatorname{Ind}\left(T_{k-l, 0}\right)$. Tomando $n:=k-l$, resta então provar esta proposição para $T_{n, 0}$. Para isto, vamos calcular a dimensão do núcleo e a codimensão da imagem do operador abaixo:

$$
T_{n, 0}=M_{\chi^{n}} D_{b}+I d-D_{b}
$$

Escrevendo $f \in L^{2}\left(S^{1}\right)$ como a sua série de Fourier $\sum_{j \in \mathbb{Z}} a_{j} z^{j}$, temos que:

$$
T_{n, 0}(f)=z^{n} \sum_{j>0} a_{j} z^{j}+\sum_{j \leq 0} a_{j} z^{j}
$$


Assim, é mais fácil analizar o que acontece com a transformação $T$, que faz o diagrama abaixo comutar:

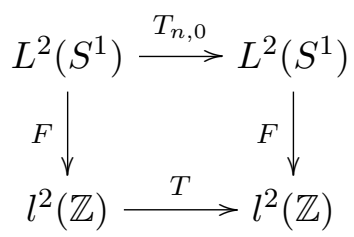

Logo, se $n \geq 0$, então $T$ associa: $\left(c_{j}\right)_{j \in \mathbb{Z}} \longmapsto(\ldots, c_{-1}, c_{0}, \underbrace{0, \ldots, 0}_{n \text { casas }}, c_{1}, c_{2}, \ldots)$.

Segue que $T$ é injetora. Logo, $\operatorname{dim}(\operatorname{ker}(T))=0$. Além disso, o conjunto:

$$
\left\{\left[e_{1}\right],\left[e_{2}\right], \ldots,\left[e_{n}\right]\right\} \subset \frac{l^{2}(\mathbb{Z})}{\operatorname{Im}(T)}
$$

forma uma base de $\frac{l^{2}(\mathbb{Z})}{\operatorname{Im}(T)}$ e $\operatorname{assim}, \operatorname{dim}\left(\frac{l^{2}(\mathbb{Z})}{\operatorname{Im}(T)}\right)=n$.

Por outro lado, se $n<0$, então $T$ associa:

$$
\left(c_{j}\right)_{j \in \mathbb{Z}} \longmapsto(\ldots, c_{n}, \underbrace{\left(c_{n+1}+c_{1}\right), \ldots,\left(c_{0}+c_{|n|}\right)}_{|n| \text { casas }}, c_{|n|+1}, \ldots) .
$$

Neste caso é claro que $T$ é sobrejetora. Seque então que $\operatorname{dim}\left(\frac{l^{2}(\mathbb{Z})}{\operatorname{Im}(T)}\right)=0$. Além disso, temos que $\operatorname{ker}(T)$ é gerado pelo conjunto:

$$
\left\{\left(e_{0}-e_{|n|}\right),\left(e_{-1}-e_{|n|-1}\right), \ldots,\left(e_{n+1}-e_{1}\right)\right\} ;
$$

$\operatorname{logo}, \operatorname{dim}(\operatorname{ker}(T))=|n|=-n$.

Finalmente, se $n=0$ não há o que fazer, pois neste caso temos a função identidade. Em qualquer caso,

$$
\operatorname{Ind}(T)=n .
$$

Como $F$ é isomorfismo, $\operatorname{Ind}\left(T_{n, 0}\right)=\operatorname{Ind}(T)$, e então:

$$
\operatorname{Ind}\left(T_{k, l}\right)=\operatorname{Ind}\left(T_{n, 0}\right)=\operatorname{Ind}(T)=n=k-l,
$$

como queríamos demonstrar.

Então para cada operador de Fredholm $A \in \mathcal{A}$, existem $k, l \in \mathbb{Z}$ tais que: $k=$ wind\# $\left(\sigma^{+}(A)\right)$ e $l=$ wind $\#\left(\sigma^{-}(A)\right)$, de forma que se considerarmos $T_{k, l}$ como acima teremos que $\sigma^{ \pm}\left(T_{k, l}\right)$ é homotópica a $\sigma^{ \pm}(A),{ }^{13}$ e portanto $\sigma\left(T_{k, l}\right)$

\footnotetext{
${ }^{13}$ Pois, se $f, g \in C\left(S^{1}, \mathbb{C} \backslash\{0\}\right)$, então: $f$ é homotópica a $g$ se, e só se, wind\# $(f)=$ wind\# $(g)$. Para uma demonstração disto, veja [11], página 65 .
} 
é homotópica a $\sigma(A)$. Assim, pela proposição 8.6, $\operatorname{Ind}(A)=\operatorname{Ind}\left(T_{k, l}\right)$. Segue que:

$$
\operatorname{ind}(A)=\operatorname{ind}\left(T_{k, l}\right)=k-l=\operatorname{wind} \#\left(\sigma^{+}(A)\right)-\operatorname{wind} \#\left(\sigma^{-}(A)\right),
$$

como queríamos demonstrar.

8.9. Observação. $\mathrm{O}$ valor do índice de um operador de Fredhom $A$ é dado então por duas fórmulas diferentes, cada uma delas diferença de dois inteiros, a saber: wind\# $\left(\sigma^{+}(A)\right)$ e wind\# $\left(\sigma^{-}(A)\right)$; e $\operatorname{dim}(\operatorname{ker}(A))$ e $\operatorname{codim}(\operatorname{Im}(A))$, no caso da fórmula provada acima, e da definição do índice, respectivamente. Entretanto, estes pares de inteiros não precisam coincidir. Consideremos o seguinte exemplo ${ }^{14}: M_{b} \in \mathcal{A}$, onde:

$$
\begin{aligned}
b: \mathbb{Z} & \longrightarrow \mathbb{C} \\
j & \longmapsto b(j):=\frac{1-j^{2}}{1+j^{2}}
\end{aligned}
$$

Pelo lema 7.17 , temos que $\sigma\left(D_{b}\right)\left(\operatorname{aval}_{z}, \operatorname{aval}_{j}\right)=b(j)$, isto é:

$$
\sigma^{+}\left(D_{b}\right)(z)=\lim _{j \rightarrow+\infty} \frac{1-j^{2}}{1+j^{2}}=-1=\lim _{j \rightarrow-\infty} \frac{1-j^{2}}{1+j^{2}}=\sigma^{-}\left(D_{b}\right)(z) .
$$

Estas são funções constantes e, portanto, seus números de rotação são nulos.

Por outro lado, como a transformação de Fourier é um isomorfismo, a dimensão do núcleo e a codimensão da imagem de $D_{b}=F^{-1} M_{b} F$ são as mesmas de $M_{b}$. Assim, $v=\sum \alpha_{l} e_{l} \in \operatorname{ker}\left(M_{b}\right)$ se, e só se:

$$
\begin{aligned}
0 & =M_{b}(v)=M_{b}\left(\sum_{l \in \mathbb{Z}} \alpha_{l} e_{l}\right)=\sum_{l \in \mathbb{Z}} \alpha_{l} M_{b}\left(e_{l}\right) \\
& =\sum_{l \in \mathbb{Z}}\left(\alpha_{l} \frac{1-l^{2}}{1+l^{2}}\right) e_{l} \\
& \Leftrightarrow \forall l \in \mathbb{Z}: \alpha_{l} \frac{1-l^{2}}{1+l^{2}}=0 \\
& \Leftrightarrow \forall l \in \mathbb{Z} \backslash\{-1,1\}, \alpha_{l}=0 .
\end{aligned}
$$

$\operatorname{Logo}, \operatorname{ker}\left(M_{b}\right)=\left[\left\{e_{-1}, e_{1}\right\}\right] ;$ ou seja, $\operatorname{dim}\left(\operatorname{ker}\left(D_{b}\right)\right)=\operatorname{dim}\left(\operatorname{ker}\left(M_{b}\right)\right)=2$, e isto basta para concluir o que foi observado acima. (Usando a fórmula e a definição de índice, concluímos que a codimensão da imagem de $D_{b}$ é 2.)

\footnotetext{
${ }^{14}$ Este exemplo foi fornecido pelo Professor Ruidival, logo depois da defesa desta dissertação.
} 
9. $T \in M_{n}(\mathcal{A})$ É De Fredholm Se, E Só Se, Seu Símbolo É Inversível

Na seção anterior vimos que um operador em $\mathcal{A} \subset \mathcal{B}(\mathcal{H})$, onde $\mathcal{H}:=$ $L^{2}\left(S^{1}\right)$, era de Fredholm se, e só se, seu símbolo era uma função inversível. Nesta seção vamos definir o símbolo de um operador $T \in M_{n}(\mathcal{A}) \subset \mathcal{B}\left(\mathcal{H}^{n}\right)$, $\sigma_{n}(T) \in M_{n}\left(C\left(S^{1} \times\{+\infty,-\infty\}\right)\right)$, e mostrar que o determinante desse símbolo é inversível se, e só se, o operador $T$ é de Fredholm.

9.1. Lema. Seja $\mathcal{H}$ um espaço de Hilbert. Então $\mathcal{K}\left(\mathcal{H}^{n}\right)=M_{n}(\mathcal{K}(\mathcal{H}))$.

Demonstração. Sejam:

$$
\begin{aligned}
P_{i}: \mathcal{H}^{n} & \longrightarrow \mathcal{H} \\
\left(x_{1}, \ldots, x_{n}\right) & \longmapsto P_{i}(x):=x_{i},
\end{aligned}
$$

a projeção canônica da i-ésima coordenada, e

$$
\begin{aligned}
I_{i}: \mathcal{H} & \longrightarrow \mathcal{H}^{n} \\
x & \longmapsto I_{i}(x):=\left(\delta_{i j} x\right)_{j=1}^{n},
\end{aligned}
$$

a inclusão na i-ésima coordenada.

É claro que $I_{j} P_{j} \in \mathcal{B}\left(\mathcal{H}^{n}\right)$. Além disso, $\sum_{j=1}^{n} I_{j} P_{j}=I d$.

Assim, se $T \in \mathcal{B}\left(\mathcal{H}^{n}\right)$, temos que:

$$
T=I d \circ T \circ I d=\left(\sum_{i=1}^{n} I_{i} P_{i}\right) T\left(\sum_{j=1}^{n} I_{j} P_{j}\right)=\sum_{i, j=1}^{n} I_{i}\left(P_{i} T I_{j}\right) P_{j} .
$$

Tomando $T_{i j}:=P_{i} T I_{j}$, temos que a aplicação

$$
\begin{aligned}
\gamma: \mathcal{B}\left(\mathcal{H}^{n}\right) & \longrightarrow M_{n}(\mathcal{B}(\mathcal{H})) \\
T & \longmapsto \gamma(T):=\left(T_{i j}\right)_{i, j=1}^{n}
\end{aligned}
$$

é um $*$-isomorfismo.

Agora, temos que se $T$ é compacta, então, como $P_{i}$ e $I_{j}$ são limitadas, $T_{i j}=P_{i} T I_{j}$ é ainda compacta, para todos $i, j \in\{1, \ldots, n\}$.

Reciprocamente, se $T_{i j}$ é compacta, para todos $i, j \in\{1, \ldots, n\}$, então, como $T=\sum_{i=1}^{n} I_{i} T_{i j} P_{j}$, temos que $T$ é compacta.

Logo, segue a tese.

9.2. Definição. Definimos a função símbolo de um operador em $M_{n}(\mathcal{A})$ da seguinte forma:

$$
\begin{aligned}
\sigma_{n}: M_{n}(\mathcal{A}) & \longrightarrow M_{n}\left(C\left(S^{1} \times\{-\infty,+\infty\}\right)\right) \\
\left(A_{i j}\right)_{i, j=1}^{n} & \longmapsto \sigma_{n}\left(\left(A_{i j}\right)_{i, j=1}^{n}\right):=\left(\sigma\left(A_{i j}\right)\right)_{i, j=1}^{n}
\end{aligned}
$$


9.3. Proposição. A função símbolo, definida acima, é um *-homomorfismo.

Demonstração. O símbolo é claramente uma transformação linear. Além disso, ele preserva o produto, pois se $A, B \in M_{n}(\mathcal{A})$, então:

$$
\begin{aligned}
\sigma_{n}(A B) & =\sigma_{n}\left(\left(A_{i j}\right)_{i, j=1}^{n}\left(B_{i j}\right)_{i, j=1}^{n}\right) \\
& =\sigma_{n}\left(\left(\sum_{k=1}^{n} A_{i k} B_{k j}\right)_{i, j=1}^{n}\right)=\left(\sum_{k=1}^{n} \sigma\left(A_{i k}\right) \sigma\left(B_{k j}\right)\right)_{i, j=1}^{n} \\
& =\left(\sigma\left(A_{i j}\right)\right)_{i, j=1}^{n}\left(\sigma\left(B_{i j}\right)\right)_{i, j=1}^{n}=\sigma_{n}\left(\left(A_{i j}\right)_{i, j=1}^{n}\right) \sigma_{n}\left(\left(B_{i j}\right)_{i, j=1}^{n}\right) \\
& =\sigma_{n}(A) \sigma_{n}(B) .
\end{aligned}
$$

Agora vejamos que $\sigma_{n}$ preserva a involução, onde a involução considerada é a adjunção de matrizes.

Seja $A \in M_{n}(\mathcal{A})$. Então

$$
\begin{aligned}
\sigma_{n}\left(A^{*}\right) & =\sigma_{n}\left(\left(A_{i j}\right)_{i, j}^{*}\right)=\sigma_{n}\left(\left(A_{j i}^{*}\right)_{i, j}\right) \\
& =\left(\sigma\left(A_{j i}^{*}\right)\right)_{i, j}=\left(\sigma\left(A_{j i}\right)^{*}\right)_{i, j} \\
& =\left(\overline{\sigma\left(A_{j i}\right)}\right)_{i, j}={\overline{\sigma_{n}(A)}}^{t} \\
& =\sigma_{n}(A)^{*}
\end{aligned}
$$

Segue que $\sigma_{n}$ é um *-homomorfismo, como queríamos.

9.4. Observação. Como $\operatorname{Ker}(\sigma)=\mathcal{K}$ e como vimos que $M_{n}(\mathcal{K})=\mathcal{K}\left(\mathcal{H}^{n}\right)$, segue que $\operatorname{Ker}\left(\sigma_{n}\right)=\mathcal{K}\left(\mathcal{H}^{n}\right)$.

9.5. Corolário. $A \in M_{n}(\mathcal{A})$ é Fredholm se, e só se, $\sigma_{n}(A)$ é inversível. Isto é, A é Fredholm se, e só se, $\operatorname{det}\left(\sigma_{n}(A)\left(\right.\right.$ aval $_{z}$, avalj $\left.)\right) \neq 0$, para todo $(z, j) \in S^{1} \times\{-\infty,+\infty\}$.

Demonstração. Como vimos, $\sigma_{n}$ é um homomorfismo cujo núcleo é $M_{n}(\mathcal{K})$.

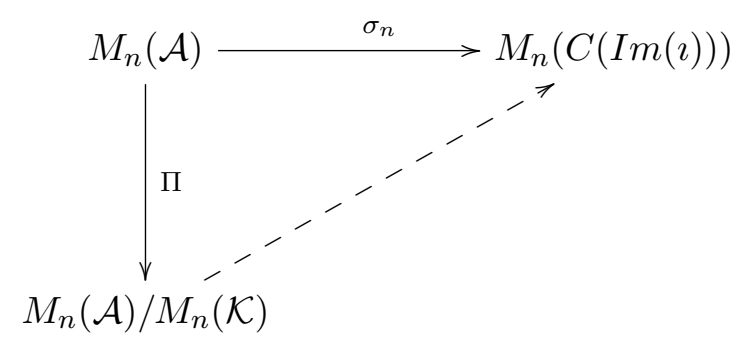


Segue do Teorema do Isomorfismo que:

$$
\frac{M_{n}(\mathcal{A})}{M_{n}(\mathcal{K})} \text { é isomorfo a } M_{n}\left(C\left(S^{1} \times\{-\infty,+\infty\}\right)\right) .
$$

Pelo Teorema de Atkinson e pelo fato de $M_{n}(\mathcal{A}) / M_{n}(\mathcal{K})$ ser uma $C^{*}$ subálgebra de $\mathcal{B}\left(\mathcal{H}^{n}\right) / \mathcal{K}\left(\mathcal{H}^{n}\right)$, temos que $T \in M_{n}(\mathcal{A})$ é de Fredholm se, e só se, $[T] \in \frac{M_{n}(\mathcal{A})}{M_{n}(\mathcal{K})}$ é inversível, pelo teorema de Atkinson. Ou seja, se, e só se, $\sigma_{n}(T) \in M_{n}\left(C\left(S^{1} \times\{-\infty,+\infty\}\right)\right)$ é inversível; o que ocorre se, e só se, $\operatorname{det}\left(\sigma_{n}(A)\left(\operatorname{aval}_{z}, \operatorname{aval} j\right)\right) \neq 0$, para todo $(z, j) \in S^{1} \times\{-\infty,+\infty\}$, como queríamos demonstrar. 
10. O Índice de $A \in \mathcal{F}\left(M_{n}(\mathcal{A})\right)$ A Partir do Símbolo de $A$

Como vimos, um operador $A \in M_{n}(\mathcal{A})$ é de Fredholm se, e só se, o seu símbolo é $\sigma_{n}(A)$ é uma função (multiplicativamente) inversível, o que ocorre se, e só se, ${ }^{15} \operatorname{det}\left(\sigma_{n}(A)(z, j)\right)=\left(\operatorname{det}\left(\sigma_{n}^{-}(A)(z, j)\right), \operatorname{det}\left(\sigma_{n}^{+}(A)(z, j)\right)\right)$ tem as duas coordenadas inversíveis, para todo $(z, j) \in \operatorname{Im}(\imath)$. Portanto, $A$ é de Fredholm se, e só se, ambas $\operatorname{det}\left(\sigma_{n}^{+}(A)\right)$ e $\operatorname{det}\left(\sigma_{n}^{-}(A)\right)$ são funções inversíveis em $S^{1}$ e que, assim, não se anulam. Logo, para um operador de Fredholm $A$, faz sentido falar no número de rotação destas funções.

O objetivo desta seção é provar a seguinte fórmula:

$$
\operatorname{Ind}(A)=\operatorname{wind} \#\left(\operatorname{det}\left(\sigma_{n}^{+}(A)\right)\right)-\operatorname{wind} \#\left(\operatorname{det}\left(\sigma_{n}^{-}(A)\right)\right) .
$$

A demonstração deste fato será quase inteiramente análoga ao que fizemos na seção anterior, com exceção de que precisaremos do seguinte lema fundamental:

10.1. Lema. Sejam $f, g \in C\left(S^{1}, G L(n, \mathbb{C})\right)$ e det $\in C\left(M_{n}(\mathbb{C})\right)$ a função determinante. Se det of e detog são homotópicas em $C\left(S^{1}, \mathbb{C} \backslash\{0\}\right)$, então $f$ e g são homotópicas em $C\left(S^{1}, G L(n, \mathbb{C})\right)$.

Demonstração. Ver apêndice E.

Por outro lado, é claro que a recíproca do lema é verdadeira, já que a função determinante é contínua.

Do que vimos no lema 9.1 , temos que $\operatorname{ker}\left(\sigma_{n}\right)=M_{n}(\mathcal{K})=\mathcal{K}\left(\mathcal{H}^{n}\right)$, que é um ideal bilateral fechado em $M_{n}(\mathcal{A})$. Além disso, a definição de $\sigma_{n}$, bem como as daquelas funções que a compõem, $\Lambda_{n}$ e $L_{n}^{-1}$, podem ser vistas como funções que aplicam a função definida no caso $n=1$ a cada uma das coordenadas das matrizes de $M_{n}(\mathcal{A})$.

Agora, vamos provar uma proposição análoga à 8.6, para podermos aplicar o Lema do Levantamento ao caso matricial também.

10.2. Proposição. Se $\sigma_{n}(T)$ é homotópica a $\sigma_{n}(S)$ no conjunto dos inversiveis de $M_{n}\left(C\left(S^{1} \times\{-\infty,+\infty\}\right)\right)$, então ind $(T)=\operatorname{ind}(S)$.

\footnotetext{
${ }^{15}$ Nesta seção identificamos $\operatorname{Im}(\imath) \operatorname{com} S^{1} \times\{-\infty,+\infty\}$ e escreveremos $(z, j)$ no lugar de $\left(\operatorname{aval}_{z}, \operatorname{aval}_{j}\right)$.
} 
Demonstração. Seja $\gamma$ uma homotopia ligando $\sigma_{n}(T)$ a $\sigma_{n}(S)$. Então, como $\Lambda_{n}^{-1} \circ L_{n}$ é um *-isomorfismo, $\Lambda_{n}^{-1} \circ L_{n} \circ \gamma$ é uma função contínua. Ou seja, uma homotopia entre $\sigma_{n}(T)$ e $\sigma_{n}(S)$ induz um caminho contínuo nos inversíveis de $M_{n}(\mathcal{A}) / M_{n}(\mathcal{K})$ ligando $[T]$ a $[S]$.

Então, tomando $\alpha:=\Lambda_{n}^{-1} \circ L_{n} \circ \gamma$ e notando que $M_{n}(\mathcal{K})$ é um ideal bilateral fechado de $M_{n}(\mathcal{A})$, pelo lema 8.5 existe $\beta$ caminho contínuo de $[0,1]$ nos inversíveis de $M_{n}(\mathcal{A})$ tal que $\beta(0)=T$ e $\beta(1)=S$. Logo, $T$ e $S$ são operadores de Fredholm unidos por uma curva de operadores de Fredholm e que portanto estão na mesma componente conexa. Como o índice é localmente constante, segue que: $\operatorname{ind}(T)=\operatorname{ind}(S)$.

Agora, só falta construir um operador $U \in \mathcal{F}\left(M_{n}(\mathcal{A})\right)$ para o qual a fórmula (2) vale. Tal construção é feita na seguinte proposição.

10.3. Proposição. Sejam $(k, l) \in \mathbb{Z}^{2}, T \in \mathcal{A}$, com wind\# $\sigma^{+}(T)=k$, wind $\# \sigma^{-}(T)=l$ (e portanto $\operatorname{Ind}(T)=k-l$ ) e $U \in M_{n}(\mathcal{A})$ definido por:

$$
U:=\left(\begin{array}{cccc}
T & & & \\
& I d & & \\
& & \ldots & \\
0 & & & I d
\end{array}\right)
$$

Então $U$ é um operador de Fredholm, $\operatorname{Ind}(U)=k-l$, wind\# $\left(\operatorname{det}\left(\sigma_{n}^{+}(U)\right)=\right.$ $k$ e wind\# $\left(\operatorname{det}\left(\sigma_{n}^{-}(U)\right)=l\right.$. Ou seja, a fórmula (2) vale para $U$.

Demonstração. Sejam $\mathcal{H}:=L^{2}\left(S^{1}\right),\left(f_{i}\right)_{i=1}^{n} \in \mathcal{H}^{n}, k^{\prime}:=\operatorname{dim} \operatorname{ker}(T)$ e $l^{\prime}:=$ $\operatorname{codim}(\operatorname{Im}(T))$. Então $U\left(\left(f_{i}\right)_{i=1}^{n}\right)=0$ se, e só se, $f_{1} \in \operatorname{Ker}(T)$ e $f_{i}=0$, para todo $i \in\{2, \ldots, n\}$. Assim, se $\mathcal{B}=\left\{v_{1}, \ldots, v_{k^{\prime}}\right\}$ é uma base de $\operatorname{Ker}(T)$, então é imediato ver que $\mathcal{C}:=\mathcal{B} \times\left\{0_{\mathcal{H}^{n-1}}\right\}$ é uma base de $\operatorname{Ker}(U)$. Logo, $\operatorname{dim}(\operatorname{Ker}(U))=k^{\prime}$.

Agora, se $\mathcal{E}=\left\{w_{1}, \ldots, w_{l^{\prime}}\right\}$ é um subconjunto L.I. de $\mathcal{H}$ tal que $[\mathcal{E}]_{T}=$ $\left\{\left[w_{1}\right]_{T}, \ldots,\left[w_{l^{\prime}}\right]_{T}\right\}$ é uma base de $\mathcal{H} / \operatorname{Im}(T)$, vejamos que $\left[\mathcal{E} \times\left\{0_{\mathcal{H}^{n-1}}\right\}\right]$ é uma base de $\mathcal{H}^{n} / \operatorname{Im}(U)$.

De fato, seja $x=\left(x_{i}\right)_{i=1}^{n} \in \mathcal{H}^{n}$. Assim, $\left[x_{1}\right]_{T}=\sum_{j=1}^{l^{\prime}} a_{j}\left[w_{j}\right]_{T}$. Logo, $x_{1}-\sum_{j=1}^{l^{\prime}} a_{j} w_{j} \in \operatorname{Im}(T)$. Por outro lado, se denotarmos os elementos de 
$\mathcal{E} \times\left\{0_{\mathcal{H}^{n-1}}\right\}$ por $\overline{w_{i}}, i \in\left\{1, \ldots, l^{\prime}\right\}$, temos que:

$$
[x]=\sum_{j=1}^{l^{\prime}} a_{j}\left[\overline{w_{j}}\right] \Leftrightarrow x-\sum_{j=1}^{l^{\prime}} a_{j} \overline{w_{j}} \in \operatorname{Im}(U) .
$$

Mas,

$$
\begin{aligned}
x-\sum_{j=1}^{l^{\prime}} a_{j} \overline{w_{l^{\prime}}} & =\left(x_{i}\right)_{i=1}^{n}-\sum_{j=1}^{l^{\prime}} a_{j}\left(w_{j}, 0, \ldots, 0\right) \\
& =\left(\left(x_{1}-\sum_{j=1}^{l^{\prime}} a_{j} w_{j}\right), x_{2}, \ldots, x_{n}\right) \in \operatorname{Im}(U) .
\end{aligned}
$$

Portanto $\left[\mathcal{E} \times\left\{0_{\mathcal{H}^{n-1}}\right\}\right]$ gera $\mathcal{H} / \operatorname{Im}(U)$.

Agora, se $\sum_{j=1}^{l^{\prime}} a_{j}\left[\overline{w_{j}}\right]=0$, então $\sum_{j=1}^{l^{\prime}} a_{j}\left[w_{j}\right]_{T}=0$. Como já sabemos que $\mathcal{B}$ é L.I., segue que $a_{j}=0$, para todo $j \in\left\{1, \ldots, l^{\prime}\right\}$.

$\operatorname{Logo}, \operatorname{dim}\left(\mathcal{H}^{n} / \operatorname{Im}(U)\right)=l^{\prime}$. Portanto, $U$ é de Fredholm e

$$
\operatorname{Ind}(U)=k^{\prime}-l^{\prime}=k-l .
$$

Vejamos agora que wind\# $\left(\operatorname{det} \circ \sigma_{n}^{+}(U)=k\right.$.

Mas isso é claro, já que $\sigma^{+}((I d))=1$ e:

$$
\operatorname{det} \circ \sigma_{n}^{+}(U)=\sigma^{+}(T) \cdot \sigma^{+}(I d) \cdot \ldots \cdot \sigma^{+}(I d)=\sigma^{+}(T) .
$$

Portanto, wind\# $\left(\operatorname{det} \circ \sigma_{n}^{+}(U)\right)=$ wind $\#\left(\sigma^{+}(T)\right)=k$ e, analogamente, wind\# $\left(\operatorname{det} \circ \sigma_{n}^{-}(U)\right)=$ wind\# $\left(\sigma^{-}(T)\right)=l$. Então temos que, de fato, a fórmula vale para $U$, pois:

$$
\operatorname{Ind}(U)=k^{\prime}-l^{\prime}=k-l=\operatorname{wind} \#\left(\operatorname{det} \circ \sigma_{n}^{+}(U)\right)-\operatorname{wind\# }\left(\operatorname{det} \circ \sigma_{n}^{-}(U)\right) .
$$

Então para cada operador de Fredholm $A \in M_{n}(\mathcal{A})$, existem $k, l \in \mathbb{Z}$ tais que: $k=$ wind\# $\left(\operatorname{det} \sigma_{n}^{+}(A)\right)$ e $l=$ wind\# $\left(\operatorname{det} \sigma_{n}^{-}(A)\right)$, de forma que se considerarmos $U$ como acima teremos que $\operatorname{det} \sigma_{n}^{ \pm}(U)$ é homotópica a $\operatorname{det} \sigma_{n}^{ \pm}(A)$. Pelo lema 10.1, isto implica que $\sigma_{n}^{ \pm}(U)$ é homotópica a $\sigma_{n}^{ \pm}(A)$; ou seja, $\sigma_{n}(U)$ é homotópica a $\sigma_{n}(A)$. Finalmente, pela proposição 10.2, $\operatorname{Ind}(A)=\operatorname{Ind}(U)$. Segue que:

$$
\begin{aligned}
\operatorname{ind}(A) & =\operatorname{ind}(U)=k-l \\
& =\operatorname{wind} \#\left(\operatorname{det}\left(\sigma_{n}^{+}(A)\right)\right)-w \operatorname{ind} \#\left(\operatorname{det}\left(\sigma_{n}^{-}(A)\right)\right) .
\end{aligned}
$$




\section{Uma Aplicação aos Operadores Diferenciais}

Fixado $m \in \mathbb{N}$, para cada $k \in\{0, \ldots, m\}$, consideremos $a_{k} \in C^{\infty}\left(S^{1}\right)$ e as funções:

$$
\begin{aligned}
b_{k}: \mathbb{Z} & \longrightarrow \mathbb{C} \\
j & \longmapsto b_{k}(j):=\frac{j^{k}}{\left(1+j^{2}\right)^{m / 2}}
\end{aligned}
$$

Então o operador:

$$
A:=\sum_{k=0}^{m} M_{a_{k}} D_{b_{k}}
$$

pertence a $\mathcal{A}$.

Além disso, como:

$$
\frac{j^{k}}{\left(1+j^{2}\right)^{m / 2}}=\frac{j^{k}}{|j|^{m}\left(1 / j^{2}+1\right)^{m / 2}} \stackrel{j \rightarrow \pm \infty}{\longrightarrow}\left\{\begin{array}{cl}
( \pm 1)^{m} & , \text { se } k=m ; \\
0 & , \text { se } k<m,
\end{array}\right.
$$

temos:

$$
\begin{aligned}
\sigma(A)\left(\operatorname{aval}_{z}, \operatorname{aval}_{ \pm \infty}\right) & =\sum_{k=0}^{m} \sigma\left(M_{a_{k}}\right)\left(\operatorname{aval}_{z}, \operatorname{aval}_{ \pm \infty}\right) \sigma\left(D_{b_{k}}\right)\left(\operatorname{aval}_{z}, \operatorname{aval}_{ \pm \infty}\right) \\
& =\sum_{k=0}^{m} a_{k}(z) b_{k}( \pm \infty) \\
& =a_{m}(z) b_{m}( \pm \infty) .
\end{aligned}
$$

Portanto, $\sigma^{+}(A)\left(\operatorname{aval}_{z}\right)=a_{m}(z)$ e $\sigma^{-}(A)\left(\operatorname{aval}_{z}\right)=(-1)^{m} a_{m}(z)$. Como $A$ é de Fredholm se, e só se, $\sigma(A)$ é multiplicativamente inversível, então, para todo $z \in S^{1}, a_{m}(z) \neq 0$ e portanto está bem definido wind\# $\left(a_{m}\right)$. Mas, para toda $f \in C(\mathbb{C} \backslash\{0\})$, wind\# $(f)=$ wind\# $(-f)$. Logo,

$$
\operatorname{Ind}(A)=\operatorname{wind} \#\left(a_{m}\right)-\operatorname{wind} \#\left((-1)^{m} a_{m}\right)=0 .
$$

Portanto, todo operador da forma (3) é de Fredholm se, e só se, $a_{m}$ nunca se anula e, neste caso, ele tem índice zero.

Consideremos agora a seguinte função:

$$
\begin{aligned}
\lambda: \mathbb{Z} & \longrightarrow \mathbb{C} \\
j & \longmapsto \lambda(j):=\frac{1}{\sqrt{1+j^{2}}},
\end{aligned}
$$

e o seu operado associado $D_{\lambda}: L^{2}\left(S^{1}\right) \longrightarrow L^{2}\left(S^{1}\right)$.

11.1. Definição. $H^{k}\left(S^{1}\right):=\operatorname{Im}\left(D_{\lambda}^{k}\right) \subset L^{2}\left(S^{1}\right)$. 
11.2. Definição. $h^{k}(\mathbb{Z}):=F\left(H^{k}\left(S^{1}\right)\right)$. Ou seja, $h^{k}(\mathbb{Z})=M_{\lambda}^{k}\left(l^{2}(\mathbb{Z})\right)$.

11.3. Proposição. $h^{k}(\mathbb{Z})=\left\{\left(a_{j}\right)_{j \in \mathbb{Z}} \in l^{2}(\mathbb{Z}):\left(j^{k} a_{j}\right)_{j \in \mathbb{Z}} \in l^{2}(\mathbb{Z})\right\}$.

Demonstração. Por um lado se $a \in h^{k}\left(S^{1}\right)$, então existe $b \in L^{2}\left(S^{1}\right)$ tal que:

$$
a_{j}=\frac{b_{j}}{\left(1+j^{2}\right)^{k / 2}}
$$

$\log 0$

$$
\left|j^{k} a_{j}\right|^{2}=\left(\frac{j^{2}}{1+j^{2}}\right)^{k}\left|b_{j}\right|^{2} \leq\left|b_{j}\right|^{2} .
$$

Por outro lado, se para cada $j \in \mathbb{Z}$ tomarmos $d_{j}:=a_{j}\left(1+j^{2}\right)^{k / 2}$, então é claro que $M_{\lambda}^{k}(d)=a$. Resta verificar que $d \in l^{2}(\mathbb{Z})$. Vejamos:

$$
\left|d_{j}\right|^{2}=\left(1+j^{2}\right)^{k}\left|a_{j}\right|^{2}=\sum_{l=0}^{k}\left(\begin{array}{l}
k \\
l
\end{array}\right) j^{2 l}\left|a_{j}\right|^{2} .
$$

Então:

$$
\sum_{j \in \mathbb{Z}}\left|d_{j}\right|^{2}=\sum_{j \in \mathbb{Z}} \sum_{l=0}^{k}\left(\begin{array}{l}
k \\
l
\end{array}\right) j^{2 l}\left|a_{j}\right|^{2} \leq \sum_{l=0}^{k}\left(\begin{array}{l}
k \\
l
\end{array}\right)\left\{\sum_{j \in \mathbb{Z}}\left|j^{k} a_{j}\right|^{2}\right\}<\infty,
$$

pois o termo entre chaves é finito por hipótese. Segue o resultado.

Podemos olhar para $H^{k}\left(S^{1}\right)$ como um espaço de Banach definindo nele a topologia induzida pela função: $D_{\lambda}^{k}: L^{2}\left(S^{1}\right) \longrightarrow H^{k}\left(S^{1}\right)$. De fato:

\subsection{Proposição.}

$$
\bigcap_{k=0}^{\infty} H^{k}\left(S^{1}\right)=C^{\infty}\left(S^{1}\right)
$$

Demonstração. Vimos na proposição 2.20 que:

$$
f \in C^{\infty}\left(S^{1}\right) \Leftrightarrow \forall k \in \mathbb{N}, \sup \left\{\left|j^{k} \widehat{f}_{j}\right|: j \in \mathbb{Z}\right\}<\infty .
$$

Além disso,

$$
\begin{aligned}
f \in H^{k}\left(S^{1}\right) & \Leftrightarrow \exists g \in L^{2}\left(S^{1}\right): D_{\lambda}^{k}(g)=f \\
& \Leftrightarrow M_{\lambda}^{k} F(g)=F(f) \\
& \Leftrightarrow \forall j \in \mathbb{Z}: \widehat{f}_{j}=\frac{\widehat{g}_{j}}{\left(1+j^{2}\right)^{k / 2}} \\
& \Leftrightarrow \forall j \in \mathbb{Z}:\left(1+j^{2}\right)^{k / 2} \widehat{f}_{j}=\widehat{g}_{j} .
\end{aligned}
$$

Suponhamos que $f \in C^{\infty}\left(S^{1}\right)$. Então, para todo $k \in \mathbb{N}, \sup \left\{\left|j^{k} \widehat{f}_{j}\right|\right\}<\infty$. Assim, fixado $k \in \mathbb{N}$, e definindo $\widehat{g}_{j}:=\left(1+j^{2}\right)^{k / 2} \widehat{f}_{j}$, é claro pela construção 
que $D_{\lambda}^{k}(g)=f$. Vejamos que a $g$ assim definida realmente pertence a $L^{2}\left(S^{1}\right)$. Tomando $l \in \mathbb{N}$, temos que:

$$
j^{l} \widehat{g}_{j}=j^{l}\left(1+j^{2}\right)^{k / 2} \widehat{f}_{j} .
$$

Então:

$$
\left(j^{l} \widehat{g}_{j}\right)^{2}=j^{2 l}\left(1+j^{2}\right)^{k} \widehat{f}_{j}^{2}=p(j) \widehat{f}_{j}^{2},
$$

onde é claro que $p \in \mathbb{C}[X]$ e só tem potências pares e seus coeficientes são positivos. Escrevendo $p(x)=\sum a_{n} x^{2 n}$ e usando que, para cada $n \in \mathbb{N}$, $M_{n}:=\sup \left\{\left|j^{n} \widehat{f}\right|: j \in \mathbb{Z}\right\}<\infty$, obtemos que:

$$
\left|j l \widehat{g}_{j}\right|^{2} \leq \sum a_{n}\left|j^{n} \widehat{f}_{j}\right|^{2}=\sum a_{n} M_{n}^{2}=: M<\infty .
$$

Portanto, para todo $l \in \mathbb{N}, \sup \left\{\left|j l \widehat{g}_{j}\right|: j \in \mathbb{Z}\right\}$ é finito. Assim, $g \in C^{\infty}\left(S^{1}\right) \subset$ $L^{2}\left(S^{1}\right)$ e segue que $f \in H^{k}\left(S^{1}\right)$, para todo $k \in \mathbb{N}$. Com isto obtemos a primeira inclusão.

Por outro lado, se $f \in \bigcap_{k=0}^{\infty} H^{k}\left(S^{1}\right)$, então, para todo $k \in \mathbb{N}$, existe $g \in L^{2}$ tal que:

$$
\forall j \in \mathbb{Z},\left(1+j^{2}\right)^{k / 2} \widehat{f}_{j}=\widehat{g}_{j}
$$

Para ver que $f \in C^{\infty}\left(S^{1}\right)$, tomemos $l \in \mathbb{N}$ e vejamos que $\sup \left\{\left|j^{l} \widehat{f}_{j}\right|: j \in \mathbb{Z}\right\}$ é finito.

De fato, basta tomar $k:=2 l$, pois então teremos:

$$
\left|j^{l} \widehat{f}_{j}\right|=\frac{\left|j^{l}\right|}{\left(1+j^{2}\right)^{k / 2}}\left|\widehat{g}_{j}\right|=\left(\frac{|j|}{1+j^{2}}\right)^{l}\left|\widehat{g}_{j}\right|<\left|\widehat{g}_{j}\right|
$$

e, como pela desigualdade de Bessel $\|\widehat{g}\|_{2} \leq\|g\|_{2}$, temos que:

$$
\left|j^{l} \widehat{f}_{j}\right|<\left|\widehat{g}_{j}\right| \stackrel{j \rightarrow \pm \infty}{\longrightarrow} 0 .
$$

Como $l$ foi tomado arbitrariamente, segue que $f \in C^{\infty}\left(S^{1}\right)$, como queríamos demonstrar.

Agora consideremos o seguinte diagrama:

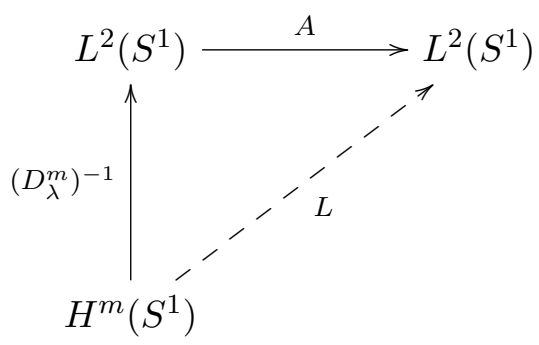


Como $\left(D_{\lambda}^{m}\right)^{-1}$ é isomorfismo de espaços de Banach, então $L$ é de Fredholm se, e só se, $A$ o é; o que ocorre se, e só se, $a_{m}\left(e^{i \theta}\right) \neq 0$, para todo $\theta \in \mathbb{R}$, e neste caso $\operatorname{Ind}(L)=0$, conforme (4).

Vimos que $C^{\infty}\left(S^{1}\right) \subset H^{m}\left(S^{1}\right)$. Assim, faz sentido perguntar: se $u \in$ $C^{\infty}\left(S^{1}\right)$, o que é $L u$ ?

11.5. Proposição. Sejam $A=\sum_{k=0}^{m} M_{a_{k}} D_{b_{k}}$ como descrita em (3), e $L:=$ $A \circ D_{\lambda}^{-m}$ que faz o diagrama acima comutar. Então:

$$
L_{\mid C^{\infty}\left(S^{1}\right)}=\sum_{k=0}^{m} a_{k}\left(\frac{1}{i} \frac{d}{d \theta}\right)^{k} .
$$

Demonstração. Seja $u \in C^{\infty}\left(S^{1}\right)$. Vejamos o que é $D_{b_{k}} \circ D_{\lambda}^{-m}(u)$ :

$$
F \circ D_{b_{k}} \circ D_{\lambda}^{-m}(u)=M_{b_{k}} \circ M_{\lambda}^{-m}(\widehat{u})
$$

Logo, para cada $j \in \mathbb{Z}$,

$$
F\left(D_{b_{k}} \circ D_{\lambda}^{-m}(u)\right)_{j}=\frac{j^{k}}{\left(1+j^{2}\right)^{m / 2}}\left(1+j^{2}\right)^{m / 2} \widehat{u}_{j}=j^{k} \widehat{u}_{j}=F\left(\left(\frac{1}{i} \frac{d}{d \theta}\right)^{k}(u)\right)_{j}
$$

Logo,

$$
A \circ D_{\lambda}^{-m}(u)=\sum_{k=0}^{m} M_{a_{k}}\left(\left(\frac{1}{i} \frac{d}{d \theta}\right)^{k}(u)\right) .
$$

Assim, para $\theta \in \mathbb{R}$,

$$
A \circ D_{\lambda}^{-m}(u)\left(e^{i \theta}\right)=\sum_{k=0}^{m} a_{k}\left(e^{i \theta}\right)\left(\frac{1}{i} \frac{d}{d \theta}\right)^{k}(u)\left(e^{i \theta}\right) .
$$

Ou seja, $L$ restrito a $C^{\infty}\left(S^{1}\right)$ é um operador diferencial linear.

Ou seja, se um operador diferencial

$$
\begin{aligned}
L_{0}: C^{\infty}\left(S^{1}\right) & \longrightarrow C^{\infty}\left(S^{1}\right) \\
u & \longmapsto L_{0}(u):=\sum_{k=0}^{m} a_{k}\left(\frac{1}{i} \frac{d}{d \theta}\right)^{k}(u)
\end{aligned}
$$

é dado, já sabemos estendê-lo a $L: H^{m}\left(S^{1}\right) \longrightarrow L^{2}\left(S^{1}\right)$, e temos o seguinte resultado:

11.6. Teorema. $L$ é de Fredholm se, e só se, para todo $\theta \in \mathbb{R}, a_{m}\left(e^{i \theta}\right) \neq 0$. Neste caso, $\operatorname{Ind}(L)=0$. 
Um operador diferencial de coeficientes em $C^{\infty}\left(S^{1}\right)$ cujo coeficiente de maior grau nunca se anula é chamado operador elíptico. Então, pelo resultado acima, $L_{0}$ é um operador elíptico em $S^{1}$ se, e só se, $L$ é de Fredholm.

Com isto fica encerrado o objetivo principal da seção. Também vale, mas não faremos a demonstração aqui, que:

11.1. Fato. $L_{0}: C^{\infty}\left(S^{1}\right) \longrightarrow C^{\infty}\left(S^{1}\right)$, da forma como foi definido acima, é de Fredholm se, e só se, $L: H^{m}\left(S^{1}\right) \longrightarrow L^{2}\left(S^{1}\right)$ é de Fredholm. E neste caso, $\operatorname{Ind}\left(L_{0}\right)=\operatorname{Ind}(L)$.

Finalmente, como afirmamos na introdução:

11.7. Proposição. A álgebra $\mathcal{A}$, definida em 3.19, é de fato a álgebra de comparação de $S^{1}, \mathcal{A}\left(S^{1}\right)$.

Demonstração. De fato, considerando a variedade compacta $S^{1}$ com a métrica riemanniana canônica, obtemos que o operador $\Delta$ definido na introdução é, neste caso, $\frac{d^{2}}{d \theta^{2}}$. Assim, $\Lambda=\left(1-\frac{d^{2}}{d \theta^{2}}\right)^{-1 / 2}$, o que, em linguagem de séries de Fourier, quer dizer que:

$$
F \circ \Lambda(f)=\left(\frac{\widehat{f}_{j}}{\left(1+j^{2}\right)^{-1 / 2}}\right)_{j \in \mathbb{Z}}=M_{\lambda} \circ F(f) .
$$

Portanto, $D_{\lambda}=\Lambda$.

Denotando:

$\mathcal{B}_{1}:=\left\{M_{a}: a \in C^{\infty}\left(S^{1}\right)\right\} \mathrm{e}$

$\mathcal{B}_{2}:=\left\{L D_{\lambda}: L\right.$ é um operador linear de ordem 1 com coeficientes $\left.C^{\infty}\right\}$

sabemos que pelas definições:

$$
\left\{\begin{aligned}
\mathcal{A} & =C^{*}\left(\mathcal{A}_{1}, \mathcal{A}_{2}\right) \mathrm{e} \\
\mathcal{A}\left(S^{1}\right) & =C^{*}\left(\mathcal{B}_{1}, \mathcal{B}_{2}\right),
\end{aligned}\right.
$$

assim, precisamos apenas mostrar que $\mathcal{B}_{1}, \mathcal{B}_{2} \subset \mathcal{A}$ e que $\mathcal{A}_{1}, \mathcal{A}_{2} \subset \mathcal{B}$. Vejamos:

$\mathcal{A}_{1} \subset \mathcal{A}\left(S^{1}\right)$, pois $\mathcal{A}_{1} \subset \overline{\mathcal{B}_{1}}$.

$\mathcal{A}_{2} \subset \mathcal{A}\left(S^{1}\right)$, pois se $L:=i \frac{d}{d \theta}$ é um operador diferencial linear de ordem 1 , então $L D_{\lambda} \in \mathcal{A}\left(S^{1}\right)$. Por outro lado, $L D_{\lambda}=D_{d}$, onde $d(j):=\frac{j}{\left(1+j^{2}\right)^{1 / 2}}$. Além disso, $D_{1}=M_{1} \in \mathcal{B}_{1} \subset \mathcal{A}\left(S^{1}\right)$. Portanto,

$$
\left\{D_{b}: b \in C^{*}(d, 1)\right\} \subset \mathcal{A}\left(S^{1}\right)
$$


Mas, $C^{*}(d, 1)=C(X)$ pelo Teorema de Stone Weierstrass. Logo, $\mathcal{A}_{2} \subset$ $\mathcal{A}\left(S^{1}\right)$.

$\mathcal{B}_{1} \subset \mathcal{A}$, pois é óbvio que $\mathcal{B}_{1} \subset \mathcal{A}_{1}$.

$\mathcal{B}_{2} \subset \mathcal{A}$, pois se $L=\frac{a_{1}}{i} \frac{d}{d \theta}+a_{0}$ é uma operador diferencial de ordem $1 \mathrm{com}$ coeficientes $C^{\infty}$, então vimos, na proposição 11.5 e observação seguinte, que $L D_{\lambda}=A=M_{a_{0}} D_{b_{0}}+M_{a_{1}} D_{b_{1}}$, e assim:

$$
\mathcal{B}_{2}=\left\{M_{a_{0}} D_{b_{0}}+M_{a_{1}} D_{b_{1}}: a_{1}, a_{2} \in C^{\infty}\left(S^{1}\right)\right\} \subset \mathcal{A},
$$

pois $M_{a_{i}} \in \mathcal{A}_{1}$ e $D_{b_{i}} \in \mathcal{A}_{2}$, para $i=0,1$. Segue que $\mathcal{A}=\mathcal{A}\left(S^{1}\right)$. 
Apêndice A. O Lema Sinal

Recordemos que definimos:

$$
\begin{aligned}
& \text { sgn }: \mathbb{Z} \longrightarrow \mathbb{C} \\
& m \longmapsto \operatorname{sgn}(m):=\left\{\begin{array}{rr}
-1, & \text { se } m<0 \\
0, & \text { se } m=0 \\
1, & \text { se } m>0 .
\end{array}\right.
\end{aligned}
$$

O objetivo desta seção é provar o seguinte resultado:

A.1. Lema. Se $a \in C^{\infty}\left(S^{1}\right)$, então $\left[M_{a}, D_{\text {sgn }}\right]$ é compacto.

Antes porém vamos precisar de alguns resultados e da definição a seguir:

A.2. Definição. Seja $S=\sum_{n \in \mathbb{Z}} a_{n} e^{n i x}$ uma série trigonométrica. Chamaremos de a série conjugada de $S$ a série $T=-i \sum_{n \in \mathbb{Z}} \operatorname{sgn}(n) a_{n} e^{i n x}$.

A.3. Notação. Seja $f \in L^{2}\left(S^{1}\right)$. Denotamos por $f^{\circ} \in L^{2}\left(S^{1}\right)$ uma função tal que $F\left(f^{\circ}\right)$ é a série conjugada de $F(f)$. Diremos então que $f^{\circ}$ é a função conjugada de $f$.

Portanto, temos que $f^{\circ}=-i D_{\text {sgn }} f$. Assim, se $a \in C^{\infty}\left(S^{1}\right)$ :

$$
\begin{aligned}
{\left[M_{a},-i D_{\text {sgn }}\right](f)\left(e^{i x}\right) } & =\left\{M_{a}\left(-i D_{\text {sgn }}\right)(f)+i D_{\text {sgn }} M_{a}(f)\right\}\left(e^{i x}\right) \\
& =\left(a f^{\circ}-(a f)^{\circ}\right)\left(e^{i x}\right) .
\end{aligned}
$$

A.4. Observação. $f \in C^{\infty}\left(S^{1}\right) \Rightarrow f^{\circ} \in C^{\infty}\left(S^{1}\right)$.

De fato, isto segue da caracterização das funções em $C^{\infty}\left(S^{1}\right)$ através das suas séries de Fourier de decrescimento rápido, proposição 2.20. Isto porque, como $\left(\widehat{f}_{j} p(j)\right)_{j \in \mathbb{Z}}$ é uma seqüência limitada, para qualquer $p \in \mathbb{C}[X]$, então é claro que a mesma seqüência com seus termos multiplicados por $-1,0$ ou 1 é ainda uma seqüência limitada.

A.5. Notação. Denotaremos $\frac{1}{2 \pi} d t$ por $d \sigma(t)$, onde $d t$ é a medida de Lebesgue na reta.

Agora, vamos usar o seguinte resultado, cuja demonstração pode ser encontrada em [8], nas páginas 122 e seguintes, para mostrar que o operador acima é compacto. 
A.6. Teorema. Seja $f \in C^{\infty}\left(S^{1}\right) .{ }^{16}$ Então, $f^{\circ}$ possui a seguinte representação, válida para todo ponto:

$$
f^{\circ}\left(e^{i x}\right)=\lim _{\varepsilon \rightarrow 0} \int_{E_{\varepsilon}} f\left(e^{i(x-t)}\right) \operatorname{cotg}(t / 2) d \sigma(t),
$$

onde $E_{\varepsilon}:=\{t \in \mathbb{R}: \varepsilon<|t|<\pi\}$.

Em vista do teorema, temos que, para toda $f \in C^{\infty}\left(S^{1}\right)$ :

$$
\begin{gathered}
-i\left[M_{a}, D_{\text {sgn }}\right](f)\left(e^{i x}\right)= \\
=\lim _{\varepsilon \rightarrow 0} \int_{E_{\varepsilon}}\left[a\left(e^{i x}\right) f\left(e^{i(x-t)}\right)-a\left(e^{i(x-t)}\right) f\left(e^{i(x-t)}\right)\right] \operatorname{cotg}(t / 2) d \sigma(t) .
\end{gathered}
$$

Definindo $\Omega_{x}^{\varepsilon}:=\{t \in \mathbb{R}: \varepsilon<|x-t|<\pi\}$ e fazendo uma mudança de variável, com $t^{\prime}:=x-t$, e em seguida fazendo $t^{\prime}:=t$, temos:

$$
\left[M_{a},-i D_{s g n}\right](f)\left(e^{i x}\right)=\lim _{\varepsilon \rightarrow 0} \int_{\Omega_{x}^{\varepsilon}}\left[a\left(e^{i x}\right)-a\left(e^{i t}\right)\right] \operatorname{cotg}\left(\frac{x-t}{2}\right) f\left(e^{i t}\right) d \sigma(t) .
$$

$\mathrm{O}$ que faremos a seguir é: obteremos um subconjunto compacto de $\mathbb{R}$ como domínio de integração e que não dependa de $\varepsilon$ para podermos passar o limite da expressão acima para o integrando, via Teorema da Convergência Dominada, após verificarmos que este se aplica aqui.

Dado que, para cada número real $u$, existe um, e apenas um, número real $q(u) \in[-\pi, \pi[$ tal que:

$$
\frac{q(u)-u}{2 \pi} \in \mathbb{Z}
$$

vamos definir a seguinte função:

$$
\begin{aligned}
q: \mathbb{R} & \longrightarrow[-\pi, \pi[ \\
u & \longmapsto q(u) .
\end{aligned}
$$

Mas integrar sobre $\Omega_{x}^{\varepsilon}$ é o mesmo que integrar sobre $q\left(\Omega_{x}^{\varepsilon}\right)=: A_{x, \varepsilon}$, já que o integrando é periódico de período $2 \pi$ nas duas variáveis, e a transformação $q$ restrita a um conjunto contido em um intervalo de comprimento menor ou igual a $2 \pi$ preserva medida. Mas agora temos a vantagem de que:

$$
\int_{\Omega_{x}^{\varepsilon}}\left[a\left(e^{i x}\right)-a\left(e^{i t}\right)\right] \operatorname{cotg}\left(\frac{x-t}{2}\right) f\left(e^{i t}\right) d \sigma(t)=
$$

\footnotetext{
${ }^{16} \mathrm{~A}$ condição original de que $f$ fosse contínua e satisfizesse uma condição de Lipschitz é sempre satisfeita por funções em $C^{\infty}\left(S^{1}\right)$, uma decorrência o Teorema Fundamental do Cálculo.
} 


$$
=\int_{-\pi}^{\pi}\left[a\left(e^{i x}\right)-a\left(e^{i t}\right)\right] \operatorname{cotg}\left(\frac{x-t}{2}\right) f\left(e^{i t}\right) \chi_{A_{x, \varepsilon}}(t) d \sigma(t),
$$

como queríamos.

Mais abaixo veremos que a expressão $\left[a\left(e^{i x}\right)-a\left(e^{i t}\right)\right] \operatorname{cotg}\left(\frac{x-t}{2}\right)$ define uma função em $\mathbb{R}^{2}$, a menos de um conjunto de medida nula, a qual possui uma única extensão em $C^{\infty}\left(\mathbb{R}^{2}\right)$. Assim, a fortiori,

$$
\left[a\left(e^{i x}\right)-a\left(e^{i t}\right)\right] \operatorname{cotg}\left(\frac{x-t}{2}\right) \chi_{A_{x, \varepsilon}}(t)
$$

tem quadrado integrável em $[-\pi, \pi]^{2} \subset \mathbb{R}^{2}$.

Para ver isso, vamos precisar do seguinte:

A.7. Lema. Seja $\phi \in C^{\infty}(\mathbb{R})$. Então a função $f: \mathbb{R}^{2} \longrightarrow \mathbb{R}$ definida por:

$$
f(x, y)= \begin{cases}\frac{\phi(x)-\phi(y)}{x-y} & , \text { se } x \neq y \\ \phi^{\prime}(x) & \text {, se } x=y\end{cases}
$$

pertence $a C^{\infty}\left(\mathbb{R}^{2}\right)$.

Demonstração. Para todo $t \in[0,1]$ temos que:

$$
\frac{d}{d t} \phi((1-t) y+t x)=\phi^{\prime}((1-t) y+t x)(x-y) .
$$

Assim, integrando nos dois membros obtemos:

$$
\int_{0}^{1} \frac{d}{d t} \phi((1-t) y+t x) d t=\int_{0}^{1} \phi^{\prime}((1-t) y+t x)(x-y) d t .
$$

Agora, usando o Teorema fundamental do Cálculo no primeiro termo temos:

$$
\phi(x)-\phi(y)=(x-y) \int_{0}^{1} \phi^{\prime}((1-t) y+t x) d t ;
$$

ou seja, se $x \neq y$, então:

$$
\frac{\phi(x)-\phi(y)}{x-y}=\int_{0}^{1} \phi^{\prime}((1-t) y+t x) d t .
$$

Portanto, $f(x, y)=\int_{0}^{1} \phi^{\prime}((1-t) y+t x) d t$, se $x \neq y$.

Mas, por outro lado, se $x=y$, então

$$
\phi^{\prime}(x)=\int_{0}^{1} \phi^{\prime}((1-t) x+t x) d t=\int_{0}^{1} \phi^{\prime}((1-t) y+t x) d t ;
$$

ou seja, $f(x, y)=\int_{0}^{1} \phi^{\prime}((1-t) y+t x) d t$, para todo $(x, y) \in \mathbb{R}^{2}$. Como o integrando é uma função $C^{\infty}$ nas três variáveis, em particular, podemos derivar indefinidamente sob o sinal de integração em relação a $x$ e a $y$, já 
que a derivada parcial do integrando será sempre uma função $C^{\infty}$. Logo, segue a tese.

A.8. Observação. Deste resultado segue, em particular, que $g: \mathbb{R} \longrightarrow \mathbb{R}$, dada pela regra de associação:

$$
g(x)= \begin{cases}\frac{\operatorname{sen}(x)}{x} & , \text { se } x \neq 0 \\ 1 & , \text { se } x=0\end{cases}
$$

é uma função $C^{\infty}$ em $\mathbb{R}$. Para ver isto, basta usar o lema, fazendo $\phi:=$ sen, e observar que $g(x)=f(x, 0)$, já que $\operatorname{sen}^{\prime}(0)=1$.

Como $g$ é uma função que não se anula em $]-\pi, \pi\left[\right.$, definindo $h:=g_{\left.\right|_{-\pi, \pi}[}$, temos que $1 / h$ existe e é também uma função de classe $C^{\infty}$.

Logo, como $\tilde{a}$ e cotg são funções de classe $C^{\infty}(\mathbb{R} \backslash\{2 k \pi: k \in \mathbb{Z}\})$ de período $\pi$,

$$
f(x, y)=[\tilde{a}(x)-\tilde{a}(y)] \operatorname{cotg}\left(\frac{x-y}{2}\right)
$$

pode ser estendida a uma função de classe $C^{\infty}$ em todo o plano, pois sendo tal função periódica de período $2 \pi$ em cada variável, em um ponto arbitrário da forma $(x, x+2 k \pi)$ - em que a fórmula acima não está definida - o valor da função estendida será o mesmo que em $(x, x)$. Ou seja, para ver que a função definida em $\mathbb{R}^{2} \backslash\{(x, x+2 k \pi): x \in \mathbb{R}$ e $k \in \mathbb{Z}\}$ pela fórmula acima pode ser estendida para uma função em $C^{\infty}\left(\mathbb{R}^{2}\right)$, basta ver que conseguimos estender esta função para os pontos da forma $(x, y)$, com $x=y$.

Se $x \neq y$, então podemos reescrever a fórmula acima como:

$$
\frac{\tilde{a}(x)-\tilde{a}(y)}{x-y}(x-y) \operatorname{cotg}\left(\frac{x-y}{2}\right)=\frac{\tilde{a}(x)-\tilde{a}(y)}{x-y} \frac{\frac{x-y}{2}}{\operatorname{sen}\left(\frac{x-y}{2}\right)} 2 \cos \left(\frac{x-y}{2}\right) .
$$

Agora, sabemos pelo lema que o primeiro fator deste produto é uma função que se estende para $C^{\infty}\left(\mathbb{R}^{2}\right)$. O terceiro fator é uma função $C^{\infty} \mathrm{em}$ $\mathbb{R}^{2}$. Quanto ao segundo fator, se $|x-y|<\pi$, então $-\pi<(x-y) / 2<\pi$, assim podemos fazer a composição $1 / h\left(\frac{x-y}{2}\right)$, que é claramente $C^{\infty}$ em uma vizinhança de $\{(x, x): x \in \mathbb{R}\}$, pois vimos que $1 / h$ é $C^{\infty}$.

Já sabíamos que $f \in C^{\infty}\left(\mathbb{R}^{2} \backslash\{(x, x+2 k \pi): x \in \mathbb{R}\right.$ e $\left.k \in \mathbb{Z}\}\right)$. Agora, pelo argumento acima, vimos que $f$ possui uma extensão em $C^{\infty}\left(\mathbb{R}^{2} \backslash\{(x, x+\right.$ $2 k \pi): x \in \mathbb{R}$ e $\left.k \in \mathbb{Z}^{*}\right\}$ ). Mas então pela periodicidade da $f$, temos que 
ela possui uma extensão $G$ para $C^{\infty}\left(\mathbb{R}^{2}\right)$, que obedece a seguinte regra de associação:

$$
G(x, y)= \begin{cases}{[\tilde{a}(x)-\tilde{a}(y)] \operatorname{cotg}\left(\frac{x-y}{2}\right)} & , \text { se } x \neq y \\ 2 \tilde{a}^{\prime}(x) & , \text { se } x=y\end{cases}
$$

Então, em particular, $G$ é contínua em $\mathbb{R}^{2}$ e portanto qualquer restrição de $G$ a um compacto $K$ de $\mathbb{R}^{2}$ pertence a $L^{p}(K)$, com $1 \leq p \leq \infty$, já que todo compacto de $\mathbb{R}^{n}$ é mensurável de medida finita, pois é fechado e limitado. (Por ser fechado, $K$ é um Boreliano e portando mensurável. Por ser limitado, $K$ tem medida finita.) Tomando $\tilde{H}(x, t):=G(x, t) \chi_{[-\pi, \pi]}(t)$ e notando que: $|G(x, \cdot) \tilde{f}| \chi_{A_{x, \varepsilon}} \leq|G(x, \cdot) \tilde{f}| \chi_{[-\pi, \pi]}$, que é integrável, pois é limitada, segue, pelo Teorema da Convergência Dominada, que:

$$
\begin{aligned}
-i\left[M_{a}, D_{\text {sgn }}\right](f)\left(e^{i x}\right) & =\lim _{\varepsilon \rightarrow 0} \int_{-\pi}^{\pi} G(x, t) \chi_{A_{x, \varepsilon}} \tilde{f}(t) d \sigma(t) \\
& =\int_{-\pi}^{\pi} G(x, t) \tilde{f}(t)\left(\lim _{\varepsilon \rightarrow 0} \chi_{A_{x, \varepsilon}}\right) d \sigma(t) \\
& =\int_{-\pi}^{\pi} G(x, t) \tilde{f}(t) \chi_{[-\pi, \pi]}(t) \frac{d t}{2 \pi} \\
& =\frac{1}{2 \pi} \int_{-\pi}^{\pi} \tilde{H}(x, t) \tilde{f}(t) d t \\
& =\int_{S^{1}} H(z, w) f(w) d \mu(w),
\end{aligned}
$$

onde $z:=e^{i x}$ e $w:=e^{i t}$. Portanto, $-i\left[M_{a}, D_{\text {sgn }}\right]$ é um operador integral com núcleo em $H \in L^{2}\left(S^{1} \times S^{1}\right)$, pois $\tilde{H} \in L^{2}\left([-\pi, \pi]^{2}\right)$ e, pelo seguinte teorema, este é um operador compacto.

A.9. Teorema. Sejam $(X, A, \mu)$ e $(Y, B, \lambda)$ espaços de medidas $\sigma$-finitas $e$ $K \in L^{2}(X \times Y)$, onde o espaço produto tem a medida produto. Então a seguinte transformação:

$$
\begin{aligned}
& T_{K}: L^{2}(Y) \longrightarrow L^{2}(X) \\
& f \longmapsto T(f): X \longrightarrow \mathbb{C} \\
& x \longmapsto T_{K}(f)(x):=\int_{Y} K(x, y) f(y) d \lambda(y),
\end{aligned}
$$

está bem definida, é contínua com norma menor ou igual a $\|K\|_{2}$ e é compacta. 
Demonstração. Usando a desigualdade de Cauchy-Schwartz e em seguida o teorema de Fubini, obtemos:

$$
\begin{aligned}
\left\|T_{K}(f)\right\|_{2}^{2} & =\int_{X}\left|T_{K}(f)(x)\right|^{2} d \mu(x) \\
& =\int_{X}\left|\int_{Y} K(x, y) f(y) d \lambda(y)\right|^{2} d \mu(x) \\
& \leq \int_{X}\left(\int_{Y}|K(x, y)|^{2} d \lambda(y) \int_{Y}|f(y)|^{2} d \lambda(y)\right) d \mu(x) \\
& =\|f\|_{2}^{2} \int_{X}\left[\int_{Y}|K(x, y)|^{2} d \lambda(y)\right] d \mu(x) \\
& =\|f\|_{2}^{2} \int_{X \times Y}|K(x, y)|^{2} d(\lambda \times \mu)(x, y) \\
& =\|f\|_{2}^{2}\|K(x, y)\|_{2}^{2}<\infty .
\end{aligned}
$$

Então, $T_{K}(f) \in L^{2}(X)$ e portanto está bem definida. Mais do que isso, $T_{K}$ é uma transformação linear limitada em $L^{2}(Y)$ cuja norma é menor ou igual a $\|K\|_{2}$, o que prova a primeira afirmação feita.

Vamos considerar a seguinte função:

$$
\begin{aligned}
\phi: L^{2}(X \times Y) & \longrightarrow \mathcal{B}\left(L^{2}(Y), L^{2}(X)\right) \\
K & \longmapsto \phi(K):=T_{K} .
\end{aligned}
$$

De imediato, temos que $\phi$ é contínua, pois, como vimos acima, $\|\phi(K)\|=$ $\left\|T_{K}\right\| \leq\|K\|_{2}$; e disso decorre que: $\|\phi\| \leq 1$. Então, se $D$ é um subconjunto denso de $L^{2}(X \times Y)$, temos que $\phi(D)$ é denso em $\phi\left(L^{2}(X \times Y)\right)$.

Vamos agora considerar a álgebra $R$ gerada pelas reuniões finitas de retângulos do tipo: $A \times B$, onde $A \subset X$ e $B \subset Y$ são conjuntos mensuráveis de medida finita. (Notemos que a medida produto restrita a $R$ é ainda $\sigma$-finita e que $\chi_{A \times B}(x, y)=\chi_{A}(x) \chi_{B}(y)$.) A proposição 4.2 .8 de [7] nos garante que:

$$
A:=\left\{\sum_{i=1}^{n} \beta_{i} \chi_{A_{i}}(x) \chi_{B_{i}}(y): n \in \mathbb{N},\left(A_{i}, B_{i}\right) \in R \text { e } \beta_{i} \in \mathbb{R}\right\}
$$

é denso em $L^{2}(X \times Y)$.

Seja $\left(A_{n}\right)_{n \in \mathbb{N}}$ é uma seqüência de funções em $A$ que converge para $K$ em $L^{2}(X \times Y)$. Então, como $\phi$ é contínua, $\left(\phi\left(A_{n}\right)\right)_{n \in \mathbb{N}}$ uma seqüência que 
converge para $\phi(K)$. Mas, $\phi\left(A_{n}\right)=T_{A_{n}}$, e

$$
\begin{aligned}
T_{A_{n}}(f)(x) & =\int_{Y}\left(\sum_{i=1}^{l} \beta_{i} \chi_{A_{i}}(x) \chi_{B_{i}}(y)\right) f(y) d \lambda(y) \\
& =\sum_{i=1}^{l} \beta_{i}\left(\int_{Y} \chi_{B_{i}}(y) f(y) d \lambda(y)\right) \chi_{A_{i}}(x) \\
& =: \sum_{i=1}^{l}\left(\beta_{i} \alpha_{i}\right) \chi_{A_{i}}(x) .
\end{aligned}
$$

Logo, $T_{K}$ é compacto, pois é limite dos operadores $T_{A_{n}}$ que têm posto finito.

Então, de fato, para toda $a \in C^{\infty}\left(S^{1}\right),\left[M_{a}, D_{\text {sgn }}\right]$ é compacto. Isto completa o objetivo deste apêndice. 
Apêndice B. O Levantamento de um Caminho em $\mathcal{A} / \mathcal{J}$

B.1. Definição. Sejam $\Omega$ um espaço Hausdorff localmente compacto e $E$ um espaço de Banach. Dizemos que $f \in C(\Omega, E)$ se anula no infinito se, e só se, para cada $\varepsilon>0$ existe $K \subset \Omega$ compacto tal que: $\|f(x)\|<\varepsilon$, se $x \notin K$.

B.2. Notação. $C_{0}(\Omega, E):=\{f \in C(\Omega, E): f$ se anula no infinito $\}$

B.3. Observação. A exigência de que $\Omega$ seja um espaço Hausdorff localmente compacto serve apenas para garantir que $C_{0}(\Omega, E) \neq\{0\}$. Isto porque todo espaço localmente compacto Hausdorff possui uma compactificação de Alexandrov; isto é $\Omega$ é homeomorfo a um subespaço denso em um espaço compacto Hausdorff $K_{\Omega} \cong \Omega \cup\{\infty\}$. Como $K_{\Omega}$ é compacto Hausdorff, $K_{\Omega}$ é normal. Assim, se $x \in \Omega$, existe, pelo lema de Urysohn, uma função contínua $g: K_{\Omega}: \longrightarrow \mathbb{R}$ contínua tal que $g(x)=1$ e $g(\infty)=0$. Tomando $v \in E \backslash\{0\}:$

$$
\begin{aligned}
\alpha:[0,1] & \longrightarrow E \\
t & \longmapsto \alpha(t):=t v,
\end{aligned}
$$

temos que a composição $f:=\alpha \circ g_{\mid \Omega} \in C_{0}(\Omega, E)$ e $f \neq 0$.

B.4. Proposição. Seja E um espaço de Banach. Então $\left(C_{0}(\Omega, E),\|\cdot\|_{\infty}\right)$ também é um espaço de Banach.

Demonstração. Seja $\left(f_{n}\right)_{n \in \mathbb{N}}$ uma seqüência de Cauchy em $\left(C_{0}(\Omega, E),\|\cdot\|_{\infty}\right)$. Como $E$ é completo, para cada $x \in \Omega$ existe o limite de $\left(f_{n}(x)\right)_{n \in \mathbb{N}}$; vamos denotá-lo por $f(x)$. Agora é só verificar que

$$
\begin{aligned}
f: \Omega & \longrightarrow E \\
x & \longmapsto f(x)
\end{aligned}
$$

é o limite uniforme de $\left(f_{n}\right)_{n \in \mathbb{N}}$ e que $f \in C_{0}(\Omega, E)$. Vejamos.

Dado $\varepsilon>0$, existe $n_{0} \in \mathbb{N}$ tal que, para todo $x \in \Omega,\left\|f_{n}(x)-f_{m}(x)\right\|<$ $\varepsilon / 3$, se $m, n \geq n_{0}$.

Assim, se $n \geq n_{0}$, para todo $x \in \Omega,\left\|f_{n}(x)-f(x)\right\| \leq \varepsilon / 3<\varepsilon$. Isto é, $f$ é o limite uniforme de $\left(f_{n}\right)_{n \in \mathbb{N}}$.

Além disso, como $f_{n_{0}}$ é contínua, existe um aberto $V$ tal que, para todo $y \in V,\left\|f_{n_{0}}(x)-f_{n_{0}}(y)\right\|<\varepsilon / 3$. Assim, para todo $y \in V$ :

$\|f(x)-f(y)\| \leq\left\|f(x)-f_{n_{0}}(x)\right\|+\left\|f_{n_{0}}(x)-f_{n_{0}}(y)\right\|+\left\|f_{n_{0}}(y)-f(y)\right\|<\varepsilon$. 
Logo, $f$ é contínua em todo $x \in \Omega$; isto é, $f \in C(\Omega, E)$.

Para ver que $f$ se anula no infinito, vamos tomar $\varepsilon>0$ e $g \in C_{0}(\Omega, E)$ tal que $\|f-g\|_{\infty}<\varepsilon / 2$. Como $g$ se anula no infinito, existe $K_{g, \varepsilon / 2} \subset \Omega$ compacto tal que se $x \notin K_{g, \varepsilon / 2}$, então $\|g(x)\|<\varepsilon / 2$. Mas então, se $x \notin$ $K_{g, \varepsilon / 2}$, temos que:

$$
\|f(x)\| \leq\|f(x)-g(x)\|+\|g(x)\|<\varepsilon .
$$

Logo, $f \in C_{0}(\Omega, E)$, como queríamos demonstrar.

B.5. Definição. Sejam $\Omega$ um espaço Hausdorff localmente compacto e $E$ um espaço de Banach. Dados $\phi \in C_{0}(\Omega)$ e $v \in E$, definimos o produto tensorial de $\phi$ por $v$ através da seguinte função:

$$
\begin{aligned}
\otimes: C_{0}(\Omega) \times E & \longrightarrow C_{0}(\Omega, E) \\
(\phi, v) & \longmapsto \otimes(\phi, v):=\phi \otimes v: \Omega \\
& \longrightarrow E \\
x & \longmapsto \phi \otimes v(x):=\phi(x) v .
\end{aligned}
$$

B.6. Observação. Seja $E$ um espaço normado. Então: $C_{0}((0,1), E)$ é isomorfo a $\{f \in C([0,1], E): f(0)=f(1)=0\}$, onde o isomorfismo é a associação naural que a cada $f \in C_{0}((0,1), E)$ associa a sua extensão contínua no intervalo $[0,1], \operatorname{com} f(0):=0$ e $f(1):=0$.

Mais geralmente, se $K_{\Omega} \cong \Omega \cup\{\infty\}$ é o compactificado de Alexandrov de um espaço localmente compacto Hausdorff $\Omega$, então $C_{0}(\Omega, A)$ é isomorfo a $\left\{f \in C(\Omega, A)\right.$ : existe $g \in C\left(K_{\Omega}, A\right), g(\infty)=0$ e $\left.f=g_{\mid \Omega}\right\}$.

O seguinte resultado foi adaptado de [9], lema 10.1.1.

B.7. Proposição. Sejam $\Omega$ um espaço Hausdorff localmente compacto e A uma $C^{*}$-álgebra. Então $\left[\otimes\left(C_{0}(\Omega) \times A\right)\right]$ é denso em $C_{0}(\Omega, A)$.

Demonstração. Sejam $f=g_{\mid \Omega} \in C_{0}(\Omega, A)$ e $\varepsilon>0$. Como $K_{\Omega}$ é compacto e $g$ é contínua, existe uma cobertura aberta finita de $K_{\Omega},\left(U_{i}\right)_{i=1}^{k}$, com a propriedade de que se $x, y \in U_{i}$, então $\|g(x)-g(y)\|<\varepsilon$, para todo $i \in\{1, \ldots, k\}$.

Agora, para cada $i$ fixemos $x_{i} \in U_{i}$, com a única restrição de que se $\infty \in U_{i}$, então $x_{i}:=\infty$.

Seja $\left(h_{i}\right)_{i=1}^{k}$ uma partição da unidade subordinada à cobertura $\left(U_{i}\right)_{i=1}^{k}$; isto é, para todo $i \in\{1, \ldots, k\}, h_{i} \in C\left(K_{\Omega},[0,1]\right), \operatorname{supp}\left(h_{i}\right) \subset U_{i}, 0 \leq h_{i} \leq 1$ 
e $\sum_{i=1}^{k} h_{i} \equiv 1$. Então, para todo $i \in\{1, \ldots, k\}$ e para todo $x \in K_{\Omega}$,

$$
\left\|g(x) h_{i}(x)-g\left(x_{i}\right) h_{i}(x)\right\| \leq\left\|g(x)-g\left(x_{i}\right)\right\| h_{i}(x) \leq \varepsilon h_{i}(x),
$$

pois se $x \in U_{i}$, então $\left\|g(x)-g\left(x_{i}\right)\right\| \leq \varepsilon$; e se não, $h_{i}(x)=0<\varepsilon$. Portanto, para todo $x \in K_{\Omega}$,

$$
\begin{aligned}
\left\|g(x)-\sum_{i=1}^{k} g\left(x_{i}\right) h_{i}(x)\right\| & =\left\|g(x) \sum_{j=1}^{k} h_{j}(x)-\sum_{i=1}^{k} g\left(x_{i}\right) h_{i}(x)\right\| \\
& =\left\|\sum_{i=1}^{k} g(x) h_{i}(x)-g\left(x_{i}\right) h_{i}(x)\right\| \\
& \leq \sum_{i=1}^{k}\left\|g(x) h_{i}(x)-g\left(x_{i}\right) h_{i}(x)\right\| \\
& \leq \sum_{i=1}^{k} \varepsilon h_{i}(x)=\varepsilon .
\end{aligned}
$$

Tomando $a_{i}:=g\left(x_{i}\right)$, segue que $\sum_{i=1}^{k} h_{i} \otimes a_{i} \in C_{0}(\Omega, A)$ já que $a_{i}:=0$, se $\infty \in U_{i}$. Então temos que:

$$
\left\|f(x)-\sum_{i=1}^{k} h_{i}(x) a_{i}\right\| \leq \varepsilon,
$$

para todo $x \in \Omega$. Logo, segue a tese.

B.8. Lema. Sejam A uma $C^{*}$-álgebra, $J$ um ideal bilateral fechado de $A e$ $\Pi: A \longrightarrow A / J$ a aplicação quociente. Então a função

$$
\begin{aligned}
H: C_{0}((0,1), A) & \longrightarrow C_{0}((0,1), A / J) \\
f & \longmapsto H(f):=\Pi \circ f
\end{aligned}
$$

é sobrejetora.

Demonstração. Como $H$ é um homomorfismo de $C^{*}$-álgebras e a imagem de um homomorfismo de $C^{*}$-álgebras é sempre fechada ${ }^{17}$, resta verificar que $\operatorname{Im}(H)$ é densa em $C_{0}((0,1), A / J)$.

De fato, como $J$ é um ideal bilateral fechado já sabemos que $A / J$ é uma $C^{*}$-álgebra. Então, pelo que vimos, $\left[\otimes\left(C_{0}(0,1) \times A / J\right)\right]$ é denso em $C_{0}((0,1), A / J)$.

\footnotetext{
${ }^{17}$ Ver [13], página 80.
} 
Seja $\phi \otimes \Pi(v) \in \otimes\left(C_{0}(0,1) \times A / J\right)$. É claro que $H(\phi \otimes v)=\phi \otimes \Pi(v)$. Logo, $\sum_{i=1}^{k} \phi_{i} \otimes \Pi\left(v_{i}\right)=H\left(\sum_{i=1}^{k} \phi_{i} \otimes v_{i}\right) \in \operatorname{Im}(H)$. Ou seja, $\operatorname{Im}(H)$ é densa em $C_{0}((0,1), A / J)$, como queríamos demonstrar.

A idéia para a demonstração do seguinte resultado foi tirada da proposição 3.4.6, de [2].

B.9. Teorema. Sejam A uma $C^{*}$-álgebra, $J$ um ideal bilateral fechado de $A$ $e \Pi: A \longrightarrow A / J$ a aplicação quociente. Sejam $x, y \in A$ e $\alpha:[0,1] \longrightarrow A / J$ um caminho contínuo ligando $[x]$ a $[y]$. Então existe um caminho contínuo $\beta:[0,1] \longrightarrow$ A tal que $\beta(0)=x, \beta(1)=y$ e $\Pi \circ \beta=\alpha$.

Demonstração. Suponhamos inicialmente que $x=y=0$. Neste caso, temos que $\alpha \in C_{0}((0,1), A / J)$ e, pelo lema acima, existe $\beta \in C_{0}((0,1), A)$ tal que: $\beta=\Pi \circ \alpha$.

No caso geral, basta considerarmos: $\tilde{\alpha}(t):=\alpha(t)-(1-t) \alpha(0)-t \alpha(1)$, pois então $\tilde{\alpha} \in C_{0}((0,1), A / J)$ e, pelo caso anterior, existe $\tilde{\beta} \in C_{0}((0,1), A)$ tal que: $\tilde{\beta}=\Pi \circ \tilde{\alpha}$. Assim, tomando $\beta(t):=\tilde{\beta}(t)+(1-t) x+t y, \beta \in C([0,1], A)$ e é tal que: $\beta=\Pi \circ \alpha$, como queríamos demonstrar. 


\section{Apêndice C. Solução do Problema de Valor de Contorno}

Estou interessado em resolver o seguinte problema de valor de contorno:

$$
\left\{\begin{array}{c}
v-4 \pi^{2} v^{\prime \prime}=g \\
v(-\pi)=v(\pi) \\
v^{\prime}(-\pi)=v^{\prime}(\pi)
\end{array}\right.
$$

Entretanto, para facilitar as contas, resolveremos primeiro o seguinte problema auxiliar em $[0,1]$, ao invés de $[-\pi, \pi]$, que será mostrado equivalente ao primeiro mais para frente:

$$
\left\{\begin{array}{l}
u^{\prime \prime}-u=f \\
u(0)=u(1) \\
u^{\prime}(0)=u^{\prime}(1)
\end{array}\right.
$$

Usando o método da variação das constantes, obtemos que a solução geral para esta equação é:

$$
\begin{aligned}
& u(x)=c_{1} \cosh (x)+c_{2} \sinh (x)+\int_{0}^{x} \sinh (x-t) f(t) d t \\
& u^{\prime}(x)=c_{1} \sinh (x)+c_{2} \cosh (x)+\int_{0}^{x} \cosh (x-t) f(t) d t
\end{aligned}
$$

Precisamos agora determinar quais são as constantes $c_{1}$ e $c_{2}$. Substituindo valores em (1) e (2), obtemos que:

$$
\left\{\begin{array}{l}
u(0)=c_{1} \\
u(1)=c_{1} \cosh (1)+c_{2} \sinh (1)+\int_{0}^{1} \sinh (1-t) f(t) d t \\
u^{\prime}(0)=c_{2} \\
u^{\prime}(1)=c_{1} \sinh (1)+c_{2} \cosh (1)+\int_{0}^{1} \cosh (1-t) f(t) d t
\end{array}\right.
$$

Ou seja,

$$
\left\{\begin{aligned}
(1-\cosh (1)) c_{1}-\sinh (1) c_{2} & =\int_{0}^{1} \sinh (1-t) f(t) d t \\
-\sinh (1) c_{1}+(1-\cosh (1)) c_{2} & =\int_{0}^{1} \cosh (1-t) f(t) d t
\end{aligned}\right.
$$

Resolvendo o sistema acima, obtemos:

$$
\begin{aligned}
& c_{1}=\frac{1-\cosh (1)}{2-2 \cosh (1)}\left[\int_{0}^{1} \sinh (1-t) f(t) d t+\sinh (1) \int_{0}^{1} \cosh (1-t) f(t) d t\right] \\
& c_{2}=\frac{1-\cosh (1)}{2-2 \cosh (1)}\left[\int_{0}^{1} \cosh (1-t) f(t) d t+\sinh (1) \int_{0}^{1} \sinh (1-t) f(t) d t\right]
\end{aligned}
$$


Logo,

$$
\begin{aligned}
u(x) & =\frac{1-\cosh (1)}{(2-2 \cosh (1))}\left\{\int_{0}^{1}[\sinh (1-t)+\cosh (1-t)] f(t) d t\right. \\
& \left.+\sinh (1) \int_{0}^{1}[\cosh (1-t) \cosh (x)+\sinh (1-t) \sinh (x)] f(t) d t\right\} \\
& +\int_{0}^{x} \sinh (x-t) f(t) d t \\
& =\frac{1}{(2-2 \cosh (1))}\left\{(1-\cosh (1)) \int_{0}^{1} \sinh (1-t+x) f(t) d t\right. \\
& \left.+\sinh (1) \int_{0}^{1} \cosh (1-t+x) f(t) d t\right\}+\int_{0}^{x} \sinh (x-t) f(t) d t \\
& =\frac{1}{(2-2 \cosh (1))} \int_{0}^{1}[\sinh (1-t+x)-\cosh (1) \sinh (1-t+x) \\
& +\sinh (1) \cosh (1-t+x)] f(t) d t+\int_{0}^{x} \sinh (x-t) f(t) d t \\
& =\frac{1}{(2-2 \cosh (1))} \int_{0}^{1}[\sinh (1-t+x)-\sinh (-t+x)] f(t) d t \\
& +\int_{0}^{x} \sinh (x-t) f(t) d t \\
& =: \int_{0}^{1} G(x, t) f(t) d t
\end{aligned}
$$

onde

$$
G(x, t):= \begin{cases}\frac{\sinh (1-t+x)-\sinh (-t+x)}{2-2 \cosh (1)}+\sinh (x-t) & \text { se } t \leq x \\ \frac{\sinh (1-t+x)-\sinh (-t+x)}{2-2 \cosh (1)} & \text { se } x \leq t\end{cases}
$$

Como ambas as expressões acima coincidem nos pontos em que $x=t$, temos que $G$ é contínua em $[0,1] \times[0,1]$. Logo, $G \in L^{2}([0,1] \times[0,1])$ Além disso, uma simples verificação mostra que, para todo $(x, t) \in[0,1] \times[0,1]$, $G(x, t)<0$. Passemos às contas:

Antes, vamos isolar duas observações úteis. Denotando $w:=-t+x$,

(1) $2-2 \cosh (1)<0$; e

(2) $\sinh (1+w)-\sinh (w)>0$, sempre. 
De fato, (2) vale, pois:

$$
\begin{aligned}
\sinh (1+w)>\sinh (w) & \Leftrightarrow e^{1+w}-e^{-1-w}>e^{w}-e^{-w} \\
& \Leftrightarrow e^{-w}\left(1-e^{-1}\right)>e^{w}(1-e) \\
& \Leftrightarrow e^{2 w}>\frac{1-e^{-1}}{1-e}=-e^{-1}
\end{aligned}
$$

Logo,

$$
\frac{\sinh (1-t+x)-\sinh (-t+x)}{2-2 \cosh (1)}<0
$$

vale sempre.

Agora vamos verificar que $G$ também é negativo para $t \leq x$. Para isto, basta verificar que:

$$
\sinh (1+w)-\sinh (w)+(2-2 \cosh (1)) \sinh (w)>0
$$

De fato, pela fórmula do seno hiprebólico da soma, e $\operatorname{como} \cosh (w)>$ $\sinh (w)$,

$(5)=\sinh (1) \cosh (w)+(1-\cosh (1)) \sinh (w)>\sinh (w)(\sinh (1)+1-\cosh (1))$

e, para $w \geq 0, \sinh (w) \geq 0$. Então precisamos verificar que $\sinh (1)+1-$ $\cosh (1)$ é positivo. Vejamos:

$$
\begin{aligned}
\sinh (1)+1-\cosh (1)>0 & \Leftrightarrow e-e^{-1}-e-e^{-1}+2>0 \\
& \Leftrightarrow e>1
\end{aligned}
$$

Segue então que (5) é de fato positiva. Logo, $G$ é negativa, como queríamos demonstrar.

Agora, vejamos que $G$ é periódica em cada uma das suas variáveis separadamente.

$$
\begin{aligned}
G(x, 0) & =\frac{\sinh (1+x)-\sinh (x)}{2-2 \cosh (1)}+\sinh (x) \\
& =\frac{\sinh (1) \cosh (x)-\sinh (x) \cosh (1)+\sinh (x)}{2-2 \cosh (1)} \\
& =\frac{\sinh (x)-(\sinh (x) \cosh (1)-\sinh (1) \cosh (x))}{2-2 \cosh (1)} \\
& =\frac{\sinh (x)-\sinh (x-1)}{2-2 \cosh (1)} \\
& =G(x, 1)
\end{aligned}
$$




$$
\begin{aligned}
G(0, t) & =\frac{\sinh (1-t)-\sinh (-t)}{2-2 \cosh (1)} \\
& =\frac{\sinh (1-t)+\sinh (t)}{2-2 \cosh (1)} \\
& =\frac{\sinh (1) \cosh (t)-\sinh (t) \cosh (1)+\sinh (t)}{2-2 \cosh (1)} .
\end{aligned}
$$

Por outro lado,

$$
G(1, t)=\frac{\sinh (2-t)-\sinh (1-t)}{2-2 \cosh (1)}+\sinh (1-t) .
$$

Logo, denotando $C:=2-2 \cosh (1)$

$$
\begin{aligned}
C G(1, t) & =\sinh (2-t)-\sinh (1-t)+(2-2 \cosh (1)) \sinh (1-t) \\
& =\sinh (2) \cosh (t)-\sinh (t) \cosh (2) \\
& +[1-2 \cosh (1)][\sinh (1) \cosh (t)-\sinh (1) \cosh (t)] \\
& =\cosh (t)(\sinh (2)+\sinh (1)-2 \cosh (1) \sinh (1)) \\
& -\sinh (t)\left(\cosh (2)+\cosh (1)-2 \cosh ^{2}(2)\right) \\
& =\cosh (t) \sinh (1)-\sinh (t) \cosh (1)+\sinh (t) .
\end{aligned}
$$

Na última passagem usei que: $\sinh (2)=2 \cosh (1) \sinh (1)$ e que: $\cosh (2)=$ $2 \cos ^{2}(1)-1$. Como as expressões concordam, temos que: $G(0, t)=G(1, t)$.

De volta ao problema original. Estamos realmente interessados em resolver:

$$
\left\{\begin{array}{l}
v-4 \pi^{2} v^{\prime \prime}=g \\
v(-\pi)=v(\pi) \\
v^{\prime}(-\pi)=v^{\prime}(\pi)
\end{array}\right.
$$

que é o mesmo que o anterior, a menos de uma mudança de variáveis e um sinal.

De fato, fazendo as seguintes mudanças de variáveis:

$$
\begin{aligned}
{[0,1] } & \longrightarrow[-\pi, \pi] \\
x & \longmapsto 2 \pi x-\pi=: \gamma \\
t & \longmapsto 2 \pi t-\pi=: \theta,
\end{aligned}
$$

temos: $2 \pi d t=d \theta$. E definindo:

$$
\begin{aligned}
v:[-\pi, \pi] & \longrightarrow \mathbb{C} \\
\gamma & \longmapsto v(\gamma):=-u\left(\frac{\gamma+\pi}{2 \pi}\right),
\end{aligned}
$$


obtemos que: $4 \pi^{2} v^{\prime \prime}(\gamma)=u^{\prime \prime}(x)$. Logo, definindo $f(x):=g(2 \pi x-\pi)$; isto é, $g(\gamma)=f\left(\frac{\gamma+\pi}{2 \pi}\right)$, temos que:

$$
u^{\prime \prime}(x)-u(x)=f(x) \Leftrightarrow v(\gamma)-4 \pi^{2} v^{\prime \prime}(\gamma)=g(\gamma),
$$

com as respectivas condições iniciais. Logo,

$$
v(\gamma)=\frac{1}{2 \pi} \int_{-\pi}^{\pi}-G\left(\frac{\gamma+\pi}{2 \pi}, \frac{\theta+\pi}{2 \pi}\right) f\left(\frac{\theta+\pi}{2 \pi}\right) d \theta
$$

e fazendo: $\tilde{H}(\gamma, \theta):=-G\left(\frac{\gamma+\pi}{2 \pi}, \frac{\theta+\pi}{2 \pi}\right)$, teremos que $\tilde{H}$ será um núcleo positivo em $L^{2}([-\pi, \pi] \times[-\pi, \pi])$ e:

$$
v(\gamma)=\frac{1}{2 \pi} \int_{-\pi}^{\pi} \tilde{H}(\gamma, \theta) g(\theta) d \theta .
$$

Portanto, pelo lema A.9, a função que a cada $g$ associa $v$ como acima é um operador linear compacto bem definido.

Tomando $H\left(e^{i \gamma}, e^{i \theta}\right):=\tilde{H}(\gamma, \theta)$, temos que $H \in L^{2}\left(S^{1} \times S^{1}\right)$ é um núcleo positivo, e o operador acima pode ser visto como um operador compacto em $L^{2}\left(S^{1}\right)$.

Além disso, como $G$ é periódica nas duas variáveis, $\tilde{H}$ também o é, e assim, $H$ é contínua em $S^{1} \times S^{1}$, fato este que é usado na demonstração da proposição 5.8 . 


\section{ApÊNDICE D. REDES}

Quando trabalhamos no $\mathbb{R}^{n}$, podemos testar várias propriedades de um conjunto $A$ usando seqüências. Por exemplo: se $A=\bar{A}$, se $A$ é compacto, ou se a função $f: \mathbb{R}^{n} \longrightarrow \mathbb{R}^{m}$ é contínua. Mas, em espaços topológicos mais gerais, o teste por seqüências falha.

D.1. Exemplo. Seja $X=[0,1]$ com uma topologia em que um subconjunto de $A \subset X$ é declarado aberto se, e só se, $0 \notin A$ ou $X \backslash A$ é enumerável.

$X$ é Hausdorff, pois dados $x_{1}, x_{2} \in X$, com $x_{1} \neq x_{2}$, ambos não nulos, $\left\{x_{1}\right\}$ e $\left\{x_{2}\right\}$ são abertos disjuntos. Se $x_{2}=0$, então $\left\{x_{1}\right\}$ e $X \backslash\left\{x_{1}\right\}$ são abertos disjuntos.

Considere agora o subconjunto $B:=] 0,1] \subset X$ e tome uma seqüência $\left(x_{n}\right)_{n \in \mathbb{N}} \subset B$ convergente em $X$. Seja $x$ o limite desta seqüência. Vejamos que $x \in B$. Para isto basta ver que $x \neq 0$.

Mas isso é verdade, já que $X \backslash\left\{x_{n}: n \in \mathbb{N}\right\}$ é aberto que contém o zero e no qual a seqüência nunca entra.

Conclusão: [0,1] nesta topologia é seqüêncialmente fechado, mas não é fechado, pois seu complementar não é aberto.

Podemos recuperar as técnicas baseadas em seqüências com pequenos ajustes se substituirmos a noção de seqüências pela de redes.

D.2. Definição. Um conjunto dirigido é um par $(I, \preceq)$, onde $I$ é um conjunto não vazio munido de uma relação $\preceq$ tal que:

(1) $\forall \alpha \in I(\alpha \preceq \alpha)$;

(2) $\forall \alpha, \beta, \gamma \in I$ ( $\alpha \preceq \beta$ e $\beta \preceq \gamma \Rightarrow \alpha \preceq \gamma)$; e

(3) $\forall \alpha, \beta \in I, \exists \gamma \in I(\alpha \preceq \gamma$ e $\beta \preceq \gamma)$.

D.3. Definição. Uma rede em um conjunto $X$ é uma função definida em um conjunto dirigido $I$ tomando valores em $X$.

D.4. Exemplo. Toda seqüência é uma rede.

D.5. Exemplo. $\mathrm{O}$ conjunto $\mathbb{R}$ com a sua ordem natural $(x \preceq y$, se $x \leq y$ ) é um conjunto dirigido. 
D.6. Exemplo. Sejam $X$ um espaço topológico e $x \in X$. Seja $\tau_{x}$ o conjunto das vizinhanças de $x$, munido da relação $\preceq$ definida por:

$$
\forall U, V \in I(U \preceq V: \Leftrightarrow U \supset V) .
$$

Então $\left(\tau_{x}, \preceq\right)$ é um conjunto dirigido e se tomarmos $x_{U} \in U$, para cada $U$ vizinhança de $x$, então $\left(x_{U}\right)_{U \in I}$ é uma rede em $X$.

D.7. Definição. Seja $\left(x_{\alpha}\right)_{\alpha \in I}$ uma rede em um espaço topológico $X$. Dizemos que $\left(x_{\alpha}\right)_{\alpha \in I}$ é uma rede convergente em $X$ se, e só se:

$$
\exists x \in X, \forall U \in \tau_{x}, \exists \gamma \in I\left(\gamma \preceq \alpha \Rightarrow x_{\alpha} \in U\right) .
$$

Neste caso, dizemos que $\left(x_{\alpha}\right)_{\alpha \in I}$ converge para $x$ e escrevemos $x_{\alpha} \longrightarrow x$.

D.8. Definição. Sejam $X$ um conjunto e $B \subset \mathcal{P}(X)$. $B$ é uma base para uma topologia se, e só se:

(1) $\cup B=X$; e

(2) $\forall W_{1}, W_{2} \in B, \forall x \in W_{1} \cap W_{2}, \exists W_{3} \in B\left(x \in W_{3} \subset W_{1} \cap W_{2}\right)$.

Neste caso, a topologia gerada por $B$ é aquela (definida univocamente) cujos abertos são as reuniões dos elementos de $B$.

D.9. Exemplo. Consideremos o conjunto dirigido $(\mathbb{Z}, \leq)$ e a rede:

$$
\begin{aligned}
f: \mathbb{Z} & \longrightarrow \mathbb{R} \\
j & \longmapsto f(j):=\left\{\begin{aligned}
j & \text { se } j \leq 0 \\
1 / j & \text { se } 0<j .
\end{aligned}\right.
\end{aligned}
$$

Então é claro que $f$ é convergente (com limite 0 ), mas $f$ não é limitada!

D.10. Definição. Sejam $X$ o conjunto e $S B \subset \mathcal{P}(X)$. $S B$ é uma sub-base para uma topologia se, e só se, as intersecções finitas de elementos de $S B$ formam uma base.

D.11. Proposição. Sejam $X$ um espaço topológico, $x \in X, S B$ uma subbase para uma topologia em $X$ e $\left(x_{\alpha}\right)_{\alpha \in I}$ uma rede em $X$. Então $x_{\alpha} \longrightarrow x$ se, e só se, para todo $U \in S B$, com $x \in U$, existir $\gamma \in I$ tal que $x_{\alpha} \in U$, se $\gamma \preceq \alpha$.

Demonstração. Se $x_{\alpha} \longrightarrow x$, então, dado $V \in \tau_{x}$, existe $\gamma \in I$ tal que $x_{\alpha} \in V$, se $\gamma \preceq \alpha$. Em particular, como todos os elementos de $S B$ são abertos, a afirmação acima vale para $V \in \tau_{x} \cap S B$. 
Reciprocamente, se para todo $V \in S B$, com $x \in V$, existe $\gamma \in I$ tal que $x_{\alpha} \in V$, se $\gamma \preceq \alpha$, então, pela definição de conjunto dirigido, dado um número finito de elementos $U_{1}, \ldots, U_{n} \in S B \cap \tau_{x}$, existem $\left\{\gamma_{i}\right\}_{i=1}^{n} \subset I$ e $\gamma \in I$ tais que $x_{\beta} \in U_{i}$, se $\gamma_{i} \preceq \beta$, e $\gamma_{i} \preceq \gamma$, para $i \in\{1, \ldots, n\}$. Logo, para $\gamma \preceq \beta$, $x_{\beta} \in \cap_{i=1}^{n} U_{i}$. Como os abertos da topologia são justamente as reuniões de intersecções finitas de elementos de $S B$, segue da definição de convergência que $x_{\alpha} \longrightarrow x$.

D.12. Proposição. Sejam $X$ um espaço topológico, $S \subset X$ e $x \in X$. Então $x \in \bar{S}$ se, e só se, existe uma rede em $S$ que converge para $x$.

Demonstração. Se $x \notin \bar{S}$, então existe $V \in \tau_{x}$ tal que $V \cap S=\emptyset$. Logo não existe rede em $S$ convirja para x.

Reciprocamente, se $x \in \bar{S}$, então, para todo $V \in \tau_{x}$, existe $x_{V} \in V \cap S$. Considerando o conjunto dirigido $\left(\tau_{x}, \supset\right)$, segue que $\left(x_{U}\right)_{U \in \tau_{x}}$ é uma rede em $S$ que converge para $x$.

Como um subconjunto $S$ de um espaço topológico $X$ é fechado se, e só se, contém o seu próprio fecho, segue que $S$ é fechado se, e só se, $S$ contém todos os limites das redes em $S$ convergentes em $X$.

Compare este resultado com o primeiro exemplo.

D.13. Definição. Sejam $X$ e $Y$ espaços topológicos, $x \in X$ e $f: X \longrightarrow Y$ uma função. $f$ é contínua em $x$ se, e só se, para todo $U \in \tau_{f(x)}$, existe $V \in \tau_{x}$ tal que $f(V) \subset U$. $f$ é contínua se, e só se, $f$ é contínua em todo $x \in X$.

D.14. Proposição. Sejam $X$ e $Y$ espaços topológicos e $f: X \longrightarrow Y$ uma função. Então $f$ é contínua em $x \in X$ se, e só se, para toda rede $\left(x_{\alpha}\right)_{\alpha \in I}$ que converge para $x$, vale que $f\left(x_{\alpha}\right) \longrightarrow f(x)$.

Demonstração. Se $f$ é contínua, então, dado $U \in \tau_{f(x)}$, existe $V \in \tau_{x}$ tal que $f(V) \subset U$. Se $\left(x_{\alpha}\right)_{\alpha \in I}$ é uma rede que converge para $x$, então existe $\alpha \in I$ tal que se $\alpha \preceq \beta$, então $x_{\beta} \in V$. Logo, $f\left(x_{\beta}\right) \in f(V) \subset U$; ou seja, $f\left(x_{\alpha}\right)$ converge para $f(x)$.

Reciprocamente, se $f$ não é contínua em $x$, então existe $U \in \tau_{f(x)}$ tal que $f(V) \cap(Y \backslash U) \neq \emptyset$, para todo $V \in \tau_{x}$. Então tomando $x_{V} \in V$ tal que $f\left(x_{V}\right) \notin U$, temos que $\left(x_{V}\right)_{V \in \tau_{x}}$ é uma rede que converge para $x$, mas que $\left(f\left(x_{V}\right)\right)_{V \in \tau_{x}}$ não converge para $f\left(x_{0}\right)$. 
D.15. Definição. Um subconjunto $K$ de um espaço topológico $X$ é compacto se, e só se, toda cobertura de $S$ por abertos de $X$ possui uma subcobertura finita.

Se $K$ é um subconjunto de um espaço métrico $(M, d)$, então é um fato bem conhecido que $K$ é compacto se, e só se, toda seqüência em $K$ possui subseqüência convergente. Mas esta caracterização não vale para espaços topológicos em geral. Se $X$ é um espaço topológico não metrizável, então pode existir $K \subset X$ com a propriedade de que toda seqüência em $K$ possui subseqüência convergente, mas $K$ não ser compacto.

Todavia, esta caracterização possui uma análoga válida para espaços topológicos se substituirmos seqüência por rede e subseqüência por sub-rede. Antes de verificar isto, vamos, é claro, precisar das seguintes definições:

D.16. Definição. Seja $(I, \preceq)$ um conjunto dirigido. $J \subset I$ é cofinal se, e só se:

$$
\forall \alpha \in I, \exists \beta \in J(\alpha \preceq \beta) .
$$

D.17. Definição. Sejam $X$ um conjunto, $\left(I, \preceq_{I}\right)$ e $\left(J, \preceq_{J}\right)$ dois conjuntos dirigidos e $f: I \longrightarrow X$ uma rede. $h: J \longrightarrow X$ é uma sub-rede de da rede $f$ se, e só se, existe uma função $g: J \longrightarrow I$ que satisfaz:

(1) $\forall \beta_{1}, \beta_{2} \in J\left(\beta_{1} \preceq_{J} \beta_{2} \Rightarrow g\left(\beta_{1}\right) \preceq_{I} g\left(\beta_{2}\right)\right)$;

(2) $g(J)$ é cofinal em $I$; e

(3) $h=f \circ g$.

D.18. Observação. Se $(I, \preceq)$ é um conjunto dirigido e $L \subset I$ é cofinal, então $\left(L, \preceq_{\mid L \times L}\right)$ também é um conjunto dirigido.

Isto porque enquanto os dois primeiros axiomas de conjuntos dirigidos se verificam automaticamente, já que $L \subset I$, o terceiro segue da cofinalidade de $L$, pois, dados $\alpha, \beta \in L \subset I$, existe $\gamma \in I$ tal que $\alpha \preceq \gamma$ e $\beta \preceq \gamma$. Mas como $L$ é cofinal existe $\delta \in L$ tal que $\gamma \preceq \delta$. Logo, $\alpha \preceq \delta$ e $\beta \preceq \delta$; ou seja, $\left(L, \preceq_{\mid L \times L}\right)$ satisfaz os axiomas de conjunto dirigido, como queríamos demonstrar.

D.19. Observação. Se $f: I \longrightarrow X$ é uma rede, é usual denotá-la por $\left(x_{\alpha}\right)_{\alpha \in I}$, deixando a função $f$ subentendida, como já vínhamos fazendo. Da mesma forma, uma sub-rede $f \circ g$ de $f$ deveria ser representada por $((f \circ g)(\beta))_{\beta \in J}$, mas poderemos usar a notação simplificada $\left(x_{\alpha_{\beta}}\right)_{\beta \in J}$. 
D.20. Exemplo. Sejam $\left(x_{n}\right)_{n \in \mathbb{N}}$ uma seqüência em um conjunto qualquer e $[1, \infty[$ munido com a ordem usual. Consideremos a função:

$$
\begin{aligned}
g:[1, \infty[ & \longrightarrow \mathbb{N} \\
x & \longmapsto g(x):=\max \{n \in \mathbb{N}: n \leq x\} .
\end{aligned}
$$

Então $\left(x_{n_{r}}\right)_{r \in[1, \infty[}:=\left(x_{g(r)}\right)_{r \in[1, \infty[}$ é uma sub-rede da seqüência $\left(x_{n}\right)_{n \in \mathbb{N}}$. Este exemplo mostra também que a cardinalidade do conjunto de índices de uma sub-rede pode ser maior que a do conjunto de índices da própria rede.

D.21. Proposição. Sejam $X$ um espaço topológico e $\left(x_{\alpha}\right)_{\alpha \in I}$ uma rede que converge para $x \in X$. Então, toda sub-rede de $\left(x_{g(\beta)}\right)_{\beta \in J}$ converge para $x$.

Demonstração. Seja $\left(x_{g(\beta)}\right)_{\beta \in J}$ uma sub-rede de $\left(x_{\alpha}\right)_{\alpha \in I}$. Dado $V \in \tau_{x}$, existe $\gamma \in I$ tal que $x_{\alpha} \in V$, se $\gamma \preceq \alpha$. Como $g(J)$ é cofinal, existe $\delta \in g(J)$ tal que $\gamma \preceq \delta$. Seja então $\mu \in J$ tal que $g(\mu)=\delta$. Como $g$ preserva a ordem, segue que se $\mu \preceq_{J} \beta$, então $\delta=g(\mu) \preceq g(\beta)$. Logo, $x_{g(\beta)} \in V$, se $\mu \preceq_{J} \beta$. Logo, $x_{g(\beta)} \longrightarrow x$, como queríamos demonstrar.

D.22. Proposição. Sejam $X$ um espaço topológico, $x \in X$ e $\left(x_{\alpha}\right)_{\alpha \in I}$ uma rede em $X$ tal que todas as suas sub-redes de convergem para $x$. Então $x_{\alpha} \longrightarrow x$.

Demonstração. Suponhamos que não. Então existe $V \in \tau_{x}$ tal que, para todo $\alpha \in I$, existe $\gamma \in I$ tal que $\alpha \preceq \gamma$ e $x_{\gamma} \notin V$. Em vista disso, o conjunto:

$$
J:=\left\{\alpha \in I: x_{\alpha} \notin V\right\}
$$

é cofinal em $I$. Então, por D.18, $\left(x_{\alpha}\right)_{\alpha \in J}$ é uma sub-rede de $\left(x_{\alpha}\right)_{\alpha \in I}$ que não converge para $x$, o que contraria a hipótese. Logo, segue a tese.

D.23. Proposição. Sejam $X$ um espaço topológico e $S \subset X$ um subconjunto. Então $S$ é compacto se, e só se, toda rede em $S$ possui sub-rede convergente.

Demonstração. Se $\left(x_{\alpha}\right)_{\alpha \in I}$ é uma rede em $S$ que não possui sub-rede convergente, então cada ponto $x \in S$ existem $\gamma_{x} \in I$ e $V_{x} \in \tau_{x}$ tais que se $\gamma_{x} \preceq \alpha$, então $x_{\alpha} \notin V_{x}$. Vejamos que $S$ não é compacto.

Se $S$ fosse compacto, como $S \subset \cup_{x \in S} V_{x}$, então existiria $n \in \mathbb{N}$ tal que $S \subset \cup_{i=1}^{n} V_{x_{i}}$. Além disso, como $I$ é dirigido, existiria $\gamma \in I$ tal que $\gamma_{i} \preceq \gamma$, para todo $i \in\{1, \ldots, n\}$. Logo, se $\gamma \preceq \alpha$, então $x_{\alpha} \notin S$ : contradição. 
Portanto, $S$ não é compacto. Ou seja, se $S$ é compacto, então toda rede possui sub-rede convergente.

Reciprocamente, se $S$ não é compacto, então existe uma cobertura $C=$ $\left\{V_{\lambda}\right\}_{\lambda \in \Lambda}$ de $S$ por abertos, a qual não admite sub-cobertura finita. Definindo $E:=\wp_{f}(C)$, temos que, para cada $D \subset E$, existe $x_{D} \in S \backslash \cup D$. Além disso, $E$ é dirigido, pois é claro que $E$ é parcialmente ordenado pela inclusão e que, dados $D_{1}, D_{2} \in E$, existe $D=D_{1} \cup D_{2} \in E$ tal que $D_{1}, D_{2} \subset D$. Vejamos que $\left(x_{D}\right)_{D \in E}$ é uma rede em $S$ que não possui sub-rede convergente.

De fato, dado $x \in S$, existe $V \in C$ tal que $x \in V$. Como $\{V\} \in E$, temos que para todo $V \in D, x_{D} \notin \cup D$ e portanto $x_{D} \notin V$. Como uma sub-rede precisaria ser definida em um conjunto dirigido cofinal em $E$, não há sub-rede que convirja para $x$. Como $x$ foi tomado arbitrariamente, segue que esta rede não possui sub-rede convergente. 
Apêndice E. Lema de Topologia Algébrica

O objetivo deste apêndice é mostrar que se $f, g \in C\left(S^{1}, G L(n, \mathbb{C})\right)$ e det of e det $\circ g$ são homotópicas em $C\left(S^{1}, \mathbb{C} \backslash\{0\}\right)$, então $f$ e $g$ são homotópicas em $C\left(S^{1}, G L(n, \mathbb{C})\right)$. Por uma questão de simplicidade vamos adotar a seguinte: E.1. Notação. Se duas funções $f$ e $g$ forem homotópicas, escreveremos: $f \sim g$.

E.2. Notação. $G L(n, \mathbb{C}):=\left\{A \in M_{n}(\mathbb{C}): A\right.$ é inversível $\}$.

E.3. Notação. $U(n):=\left\{A \in G L(n, \mathbb{C}): A A^{*}=I d\right\}$.

E.4. Observação. Se $A \in U(n)$, então $\operatorname{det} A \in S^{1}$. De fato, como $A A^{*}=I d$, tomando o determinante dos dois lados obtemos:

$$
1=\operatorname{det}(A) \operatorname{det}\left(A^{*}\right)=\operatorname{det}(A) \overline{\operatorname{det}(A)}=|\operatorname{det}(A)|^{2} ;
$$

isto é, $|\operatorname{det}(A)|=1$.

E.5. Notação. $T_{+}(n):=\left\{\left(a_{i j}\right)_{i, j=1}^{n} \in M_{n}(\mathbb{C}): a_{i i}>0\right.$ e $a_{i j}=0$, se $\left.i>j\right\}$; ou seja, o conjunto das matrizes triangulares superiores cujas diagonais possuem todas as entradas positivas.

E.6. Lema. $U(n) \bigcap T_{+}(n)=\{I d\}$.

Demonstração. Seja $L \in U(n) \bigcap T_{+}(n)$. É fácil de ver que as colunas de uma matriz unitária formam uma base ortonormal. Assim, nenhuma das entradas de $L$ pode ter módulo maior que 1; caso contrário, a norma do vetor coluna ao qual ela pertencesse seria maior que 1 . Pela observação acima, e como $L \in T_{+}(n)$, o produto dos elementos da sua diagonal é 1 . Sendo todos os elementos da diagonal estritamente positivos, todos têm de ser 1; caso contrário teria de haver um deles maior que 1, uma contradição. Além disso, como cada vetor coluna já tem uma entrada 1, suas outras entradas têm de ser 0 ; caso contrário sua norma seria maior que 1 , uma contradição. Logo, $L=I d$, como queríamos demonstrar.

E.7. Proposição. $G L(n, \mathbb{C})$ é homeomorfo a $U(n) \times T_{+}(n)$.

Demonstração. Vamos mostrar que para cada $A \in G L(n, \mathbb{C})$, existem $U \in$ $U(n)$ e $T \in T_{+}(n)$ tais que: $A=T U$. 
Sejam $\mathcal{B}:=\left\{v_{1}, \ldots, v_{n}\right\} \subset \mathbb{C}^{n}$ os vetores-coluna da matriz $A$. Como $A$ é inversível, $\mathcal{B}$ é uma base de $\mathbb{C}^{n}$. Nesta notação então, temos que $A=[I d]_{\mathcal{B C}}$; ou seja, $A$ é a matriz de mudança de base que recebe um vetor na base $\mathcal{B}$ e o escreve na base canônica $\mathcal{C}$.

Por outro lado, podemos aplicar o processo de ortogonalização de GramSchmidt à base $\mathcal{B}$ para obter uma nova base ortonormal $\mathcal{A}$. O processo é construtivo e a fórmula para cada vetor de $\mathcal{A}:=\left\{w_{1}, \ldots, w_{n}\right\}$ é dada por:

$$
w_{i}:=\frac{x_{i}}{\left\|x_{i}\right\|}, \operatorname{com} x_{i}:=v_{i}-\sum_{j=1}^{i-1} \frac{<v_{i}, v_{j}>}{\left\|v_{j}\right\|^{2}} v_{j},
$$

para cada $i \in\{1, \ldots, n\}$. Para não ter de carregar esta expressão, vamos tomar $g_{i j}:=-\frac{<v_{i}, v_{j}>}{\left\|v_{j}\right\|^{2}}$. Assim, temos que:

$$
w_{i}=\left(\frac{g_{i 1}}{\left\|x_{i}\right\|}, \cdots, \frac{g_{i(i-1)}}{\left\|x_{i}\right\|}, \frac{1}{\left\|x_{i}\right\|}, 0, \cdots, 0\right)_{\mathcal{B}} ;
$$

ou seja,

$$
L:=[I d]_{\mathcal{A B}}=\left(\begin{array}{cccccc}
\frac{1}{\left\|x_{1}\right\|} & \frac{g_{21}}{\left\|x_{2}\right\|} & \frac{g_{3}}{\left\|x_{3}\right\|} & & & \\
0 & \frac{1}{\left\|x_{2}\right\|} & \frac{g_{32}}{\left\|x_{3}\right\|} & & & \\
0 & 0 & \frac{1}{\left\|x_{3}\right\|} & & & \\
& & & \frac{1}{\left\|x_{n-2}\right\|} & \frac{g_{(n-1)(n-2)}}{\left\|x_{n-1}\right\|} & \frac{g_{n(n-2)}}{\left\|x_{n}\right\|} \\
& & & 0 & \frac{1}{\left\|x_{n-1}\right\|} & \frac{g_{n(n-1)}}{\left\|x_{n}\right\|} \\
& & & 0 & 0 & \frac{1}{\left\|x_{n}\right\|}
\end{array}\right) \in T_{+}(n) .
$$

É fácil ver que $L^{-1}=[I d]_{\mathcal{B} \mathcal{A}} \in T_{+}(n)$. Assim,

$$
A=[I d]_{\mathcal{B C}}=[I d]_{\mathcal{A C}}[I d]_{\mathcal{B A}}=[I d]_{\mathcal{A C}} L^{-1} .
$$

Como $U:=[I d]_{\mathcal{A C}}$ é uma matriz de mudança de base entre bases ortonormais, então $U \in U(n)$. Tomando $T:=L^{-1}$, temos que $A=U T$.

Vejamos agora que a regra de associação:

$$
\begin{aligned}
\varphi: G L(n, \mathbb{C}) & \longrightarrow U(n) \times T_{+}(n) \\
A & \longmapsto \varphi(A):=(U, T)
\end{aligned}
$$

define um homeomorfismo.

De fato, $\varphi(A)=\varphi(B) \Rightarrow A=U T=B$, $\operatorname{logo} \varphi$ é injetora.

Como todas as operações envolvidas na construção do par $(U, T)$ são contínuas, $\varphi$ também o é. 
Seja $\left(U^{\prime}, T^{\prime}\right) \in U(n) \times T_{+}(n)$. Então $U^{\prime} T^{\prime} \in G L(n, \mathbb{C})$. Denotemos $A:=$ $U^{\prime} T^{\prime}, \operatorname{com} \varphi(A)=(U, T)$. Ou seja, temos então que $U^{\prime} T^{\prime}=U T$. Mas então: $U^{\prime} U^{-1}=T\left(T^{\prime}\right)^{-1} \in U(n) \bigcap T_{+}(n)$. Assim, segue pelo lema E.6 que: $U=U^{\prime}$ e $T=T^{\prime}$. Logo, $\varphi$ é sobrejetora.

Finalmente, é claro que a inversa de $\varphi$ é:

$$
\begin{aligned}
\varphi^{-1}: U(n) \times T_{+}(n) & \longrightarrow G L(n, \mathbb{C}) \\
(U, T) & \longmapsto \varphi^{-1}(U, T):=U T,
\end{aligned}
$$

que é claramente contínua, já que a multiplicação de matrizes só envolve somas e produtos das suas entradas.

E.8. Lema. $T_{+}(n)$ é convexo.

Demonstração. Sejam $A=\left(a_{i j}\right)_{i, j=1}^{n}$ e $B=\left(b_{i j}\right)_{i, j=1}^{n}$ elementos de $T_{+}(n)$. Então $(1-t) A+t B \in T_{+}(n)$, para todo $t \in[0,1]$, pois, para as entradas abaixo da diagonal o caminho é nulo; para as entradas acima da diagonal não importa; e para as coordenadas da diagonal:

$$
(1-t) a_{i i}+t b_{i i}>(1-t+t) \min \left\{a_{i i}, b_{i i}\right\}>0,
$$

para todo $t \in[0,1]$ e para todo $i \in\{1, \ldots, n\}$. Segue a tese.

E.9. Lema. Seja:

$$
\begin{aligned}
A:[0,1] & \longrightarrow G L(n, \mathbb{C}) \\
t & \longmapsto A(t)
\end{aligned}
$$

um caminho fechado e, para cada $t \in[0,1]$, vamos escrever $A(t)=U(t) T(t)$, com $U(t) \in U(n)$ e $T(t) \in T_{+}(n)$ determinadas pela construção acima. Então:

$$
\text { wind\# }(\operatorname{det}(A(t)))=\operatorname{wind} \#(\operatorname{det}(U(t))) \text {. }
$$

Demonstração. Como $T_{+}(n)$ é convexo, segue que:

$$
\begin{aligned}
H:[0,1]^{2} & \longrightarrow T_{+}(n) \\
(s, t) & \longmapsto H(s, t):=(1-s) T(t)+s I d
\end{aligned}
$$

é uma homotopia tal que: $H(0, t)=T(t)$ e $H(1, t)=I d$. Assim,

$$
\begin{aligned}
G:[0,1]^{2} & \longrightarrow G L(n, \mathbb{C}) \\
(s, t) & \longmapsto G(s, t):=U(t) H(s, t)
\end{aligned}
$$


é uma homotopia tal que: $G(0, t)=A(t)$ e $G(1, t)=U(t)$. Além disso, como $G$ é contínua em ambas as variáveis,

$$
[0,1] \ni s \longmapsto \operatorname{wind} \#(\operatorname{det} \circ G(s, \cdot)) \in \mathbb{Z}
$$

também é contínua e portanto constante. Logo, para todo $t \in[0,1]$,

$$
\begin{aligned}
\operatorname{wind} \# \circ \operatorname{det} \circ A(t) & =\text { wind\# } \circ \operatorname{det} \circ G(0, \cdot)(t) \\
& =\text { wind\# } \circ \operatorname{det} \circ G(1, \cdot)(t) \\
& =\text { wind\# } \circ \operatorname{det} \circ U(t),
\end{aligned}
$$

como queríamos demonstrar.

E.10. Notação. $S U(n):=\{A \in U(n): \operatorname{det} A=1\}$.

E.11. Proposição. A aplicação:

$$
\begin{aligned}
\Psi: U(n) & \longrightarrow S^{1} \times S U(n) \\
U & \longmapsto \Psi(U):=\left(\operatorname{det}(U), U^{\prime}\right),
\end{aligned}
$$

onde $U^{\prime}$ é a matriz $U$ com o primeiro vetor coluna dividido por $\operatorname{det}(U)$, é um homeomorfismo.

Demonstração. $\Psi$ é injetora, pois se $\Psi(U)=\left(\operatorname{det}(U), U^{\prime}\right)$, posso recuperar $U$ multiplicando a primeira coluna de $U^{\prime} \operatorname{por} \operatorname{det}(U)$.

$\Psi$ é sobrejetora, pois se $(\alpha, \tilde{U}) \in S^{1} \times S U(n)$, multiplicando a primeira coluna de $\tilde{U}$ por $\alpha$ obtemos uma matriz $U \in U(n)$. Quero mostrar que: $\Psi(U)=(\alpha, \tilde{U})$.

De fato, $U^{\prime}$ e $\tilde{U}$ são iguais a menos apenas das suas primeiras colunas, possivelmente, as quais são múltiplas escalares uma da outra, pois, tomando $\beta:=\operatorname{det}(U)$ :

$$
\beta\left(u_{1}^{\prime}, \ldots, u_{n}^{\prime}\right)=\alpha\left(\tilde{u}_{1}, \ldots, \tilde{u}_{1}\right) \Rightarrow \alpha^{-1} \beta\left(u_{1}^{\prime}, \ldots, u_{n}^{\prime}\right)=\left(\tilde{u}_{1}, \ldots, \tilde{u}_{1}\right) .
$$

Mas daí segue que:

$$
1=\operatorname{det}(\tilde{U})=\left(\alpha^{-1} \beta\right) \operatorname{det} U^{\prime}=\alpha^{-1} \beta=\alpha^{-1} \operatorname{det} U .
$$

Logo, $\operatorname{det} U=\alpha$ e, conseqüentemente, $U^{\prime}=\tilde{U}$. Ou seja, $\Psi(U)=(\alpha, \tilde{U})$.

Pelo argumento acima, fica claro que a inversa de $\Psi$ é a operação que multiplica a primeira coluna pelo escalar. Tanto $\Psi$ como $\Psi^{-1}$ são definidas por operações contínuas, portanto são, elas próprias, contínuas. Segue a tese. 
E.12. Corolário. $G L(n, \mathbb{C})$ é homeomorfo a $S^{1} \times S U(n) \times T_{+}(n)$.

E.13. Proposição. Sejam $U$ e $V$ caminhos fechados contínuos de $[0,1] \mathrm{em}$ $U(n)$. Então:

$$
\operatorname{det} \circ U \sim \operatorname{det} \circ V \Rightarrow U \sim V \text {. }
$$

Demonstração. Seja

$$
\begin{aligned}
L:[0,1]^{2} & \longrightarrow S^{1} \\
(s, t) & \longmapsto L(s, t)
\end{aligned}
$$

uma homotopia ligando $\operatorname{det}(U)$ a $\operatorname{det}(V)$; isto é, tal que: $L(0, t)=\operatorname{det} U(t)$ e $L(1, t)=\operatorname{det} V(t)$, a qual existe por hipótese.

Além disso, como $S U(n)$ é simplesmente conexo ${ }^{18}$, temos que existe outra homotopia:

$$
\begin{aligned}
I:[0,1]^{2} & \longrightarrow S U(n) \\
(s, t) & \longmapsto I(s, t)
\end{aligned}
$$

ligando $U^{\prime}$ a $V^{\prime}$; isto é, $I(0, t)=U^{\prime}(t)$ e $I(1, t)=V^{\prime}(t)$.

Assim, como $\Psi^{-1}$ é contínua, se definirmos:

$$
\begin{aligned}
H:[0,1]^{2} & \longrightarrow U(n) \\
(s, t) & \longmapsto H(s, t):=\Psi^{-1}(L(s, t), I(s, t)),
\end{aligned}
$$

obteremos que $H$ é contínua, e:

$$
H(0, t)=\Psi^{-1}(L(0, t), I(0, t))=\left(\operatorname{det}(U(t)), U^{\prime}(t)\right)=U(t) .
$$

Analogamente, $H(1, t)=V(t)$. Logo, segue que $U \sim V$, como querámos demonstrar.

E.14. Teorema. Sejam $A, B \in C\left(S^{1}, G L(n, \mathbb{C})\right)$. Se $\operatorname{det} \circ A \sim \operatorname{det} \circ B \mathrm{em}$ $C\left(S^{1}, \mathbb{C}^{*}\right)$, então $A \sim B$ em $C\left(S^{1}, G L(n, \mathbb{C})\right)$.

Demonstração. Sejam $\varphi(A)=:\left(U, T_{A}\right)$ e $\varphi(B)=:\left(V, T_{B}\right)$. Pelo lema E.9,

$$
\left\{\begin{array}{l}
\text { wind\# }(\operatorname{det} \circ A)=\text { wind\# }(\operatorname{det} \circ U), \mathrm{e} \\
\operatorname{wind} \#(\operatorname{det} \circ B)=\text { wind }(\operatorname{det} \circ V) .
\end{array}\right.
$$

Assim, como já observamos que funções em $C\left(S^{1}, \mathbb{C} \backslash\{0\}\right)$ com o mesmo número de rotação são homotópicas, temos que: $\operatorname{det} \circ A \sim \operatorname{det} \circ U$, e $\operatorname{det} \circ B \sim$ $\operatorname{det} \circ V$. Como $\operatorname{det} \circ A \sim \operatorname{det} \circ B$ por hipótese, temos que:

$$
\operatorname{det} \circ U \sim \operatorname{det} \circ A \sim \operatorname{det} \circ B \sim \operatorname{det} \circ V .
$$

\footnotetext{
${ }^{18}$ Ver [11], página 95, corolário da proposição 17.
} 
Pela proposição E.13, segue que: $U \sim V$. Além disso, vimos que $T_{+}(n)$ é convexo e portanto $T_{A} \sim T_{B}$. Tomemos então homotopias $L$ e $I$ tais que: $L(0, t)=T_{A}(t), L(1, t)=T_{B}(t), I(0, t)=U(t)$ e $I(1, t)=V(t)$. Segue então que $H(s, t):=I(s, t) L(s, t)$ é uma homotopia que liga $A$ a $B$, como queríamos demonstrar. 


\section{REFERÊNCIAS}

[1] ATIYAH, M. F., SINGER, I. M. The index of eliptic operators I. Ann. of Math. (2), v.87, p.484-530, 1968.

[2] BLACKADAR, B. K-Theory for operator Algebras. 2.ed. Cambridge. Cambridge University Press, 1998.

[3] CORDES, H. O. Elliptic Pseudo-Differential Operators - An Abstract Theory. Berlin. Springer, 1979. (Lecture Notes in Mathematics, 756).

[4] CORDES, H. O. Spectral Theory of Linear Differential Operators and Comparison Algebras. Cambridge. Cambridge University Press, 1987. (London Mathematical Society Lecture Note Series, 76).

[5] DOUGLAS, R. G. Banach Algebra Techniques in Operator Theory. 2.ed. New York: Springer, 1998. (Graduate Texts in Mathematics, 179).

[6] FEDOSOV, B.V. Index of an Eliptic System on a Manifold. Functional Analysis and Aplications, v.4, p. 312-320, 1970.

[7] FERNANDEZ, P. J. Medida e Integração. 2.ed. Rio de Janeiro: Instituto Nacional de Matemática Pura e Aplicada, 2002. (Projeto Euclides)

[8] HELSON, H. Harmonic Analysis. Pacific Grove: Wadsworth \& Brooks/Cole Advanced Books Software, 1991.

[9] LARSEn, F., LAUSTEN, N.J., RØRDAM, M. An Introduction to K-Theory for $C^{*}$-Algebras. Cambridge: Cambridge University Press, 2000.

[10] LIMA, E. L. Espaços Métricos 3.ed. Rio de Janeiro: Instituto Nacional de Matemática Pura e Aplicada, 2003. (Projeto Euclides).

[11] LIMA, E. L. Grupo Fundamental e Espaços de Recobrimento. 2.ed. Rio de Janeiro: Instituto Nacional de Matemática Pura e Aplicada, 1998. (Projeto Euclides).

[12] MELO, S. T. Norm closure of classical pseudodiferential operators does not contain Hörmander's class. Contemporary Mathematics, v. 368, p. 329-336, 2005.

[13] MURPHY, G. J. $C^{*}$-Álgebras and Operator Theory. Boston: Academic Press, 1990.

[14] RUDIN, W. Functional Analysis. New Dheli: Tata McGraw-Hill, 1973.

[15] RUDIN, W. Real and Complex Analysis. McGraw-Hill, 1970. 